Clemson University

TigerPrints

December 2020

\title{
Adaptive Protection Scheme for a Real-World Microgrid with $100 \%$ Inverter-Based Resources
}

Trupal Patel

Clemson University, trupalp@g.clemson.edu

Follow this and additional works at: https://tigerprints.clemson.edu/all_theses

\section{Recommended Citation}

Patel, Trupal, "Adaptive Protection Scheme for a Real-World Microgrid with 100\% Inverter-Based

Resources" (2020). All Theses. 3459.

https://tigerprints.clemson.edu/all_theses/3459

This Thesis is brought to you for free and open access by the Theses at TigerPrints. It has been accepted for inclusion in All Theses by an authorized administrator of TigerPrints. For more information, please contact kokeefe@clemson.edu. 
ADAPTIVE PROTECTION SCHEME FOR A REAL-WORLD MICROGRID WITH 100\% INVERTER-BASED RESOURCES

\begin{tabular}{c} 
A Thesis \\
Presented to \\
the Graduate School of \\
Clemson University \\
\hline In Partial Fulfillment \\
of the Requirements for the Degree \\
Master of Science \\
Electrical Engineering \\
\hline Trupal Patel \\
December 2020 \\
\hline Accepted by: \\
Dr. Johan Enslin \\
Dr. Ramtin Hadidi \\
\hline
\end{tabular}




\begin{abstract}
The integration of renewable and distributed energy resources to the electric power system is expected to increase, especially at the distribution level. As more renewable generation connects to distribution systems, it is imminent that existing distribution feeders will be converted to microgrids. Microgrids are systems, typically at distribution voltage level, sustained by distributed and often renewable sources, that can operate in grid-connected and islanded modes. Microgrids offer resilience by providing the flexibility of supporting the grid in normal operation and operating as self-sustained islands when the grid is disconnected.
\end{abstract}

As the grid transforms to a modular system consisting of many microgrids, these microgrids will likely evolve from existing distribution feeders, and hence will be unbalanced in nature. As the world moves towards cleaner and renewable generation, some of these microgrids may be supplied by $100 \%$ inverter-based resources (IBRs). To increase resiliency and reliability, these microgrids will need to operate in both grid-connected and islanded modes. However, inverter control and feeder protection will need to be tuned to the operating modes of the microgrid. Protection system design for microgrids pose significant challenges due to bi-directional power flow and fault currents, lower fault current due to the inverter-based resources, and wide range of fault currents during different modes of operation.

This work offers an insight into the issues involved, by taking a case study of a realworld feeder located in the southwestern US that was converted to a microgrid with three solar PV units connecting to the feeder. Different inverter control configurations and adaptive protection using different settings for different operating conditions are proposed for safe operation of this microgrid. The solution also helps to create a framework for protection and coordination of other similar microgrids. 


\section{Acknowledgements}

I would like to thank my advisor Dr. Sukumar Brahma, and my committee members, Dr. Johan H. Enslin and Dr. Ramtin Hadidi, for their support and encouraging me to pursue research. Their efforts to stimulate my curiosity, as well as to expand my knowledge have resulted in my improvement as a student and researcher. I would like to thank Matthew J. Reno and Javier Hernandez-Alvidrez from Sandia National labs for their support, knowledge and guidance thought this project. I would also like to thank my lab mates, who have provided me with ample feedback on my work, joined me in research and publishing, and pushed me to complete my thesis and explore new opportunities, while also being excellent distractions from work.

This work was funded by Sandia National Laboratories, under contract DENA0003525, Clemson PO-2004274. Sandia National Laboratories is a multimission laboratory managed and operated by National Technology \& Engineering Solutions of Sandia, LLC, a wholly owned subsidiary of Honey-well International Inc., for the U.S. Department of Energy's National Nuclear Security Administration under contract DE-NA0003525.

Clemson University is also acknowledged for generous allotment of compute time on Palmetto cluster. 


\section{Contents}

Section

Page

Title Page

Abstract $\quad$ ii

Acknowledgements

Contents $\quad$ iv

List of Figures $\quad$ vii

List of Tables $\quad$ ix

Nomenclature $\quad$ xi

1 Introduction and Literature Review 1

1.1 Inverter based resources and microgrids . . . . . . . . . . . . . . 3

1.2 Microgrid protection literature review . . . . . . . . . . . . 4

1.3 Goals . . . . . . . . . . . . . . . . . 6

2 LOHO Test System \& Modeling $\quad 7$

2.1 System overview . . . . . . . . . . . . . . . . . . . . . 7 
2.2 Lines . . . . . . . . . . . . . . . . . . . . . . . . 9

2.3 Loads . . . . . . . . . . . . . . . . . . . . . . 10

2.4 Transformers . . . . . . . . . . . . . . . . . . . 11

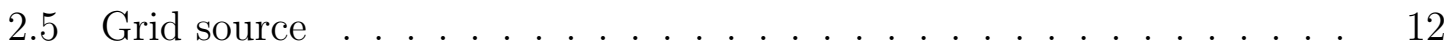

2.6 Existing protection elements in the system . . . . . . . . . . . 12

2.7 Base case steady-state \& fault simulations $\ldots \ldots \ldots \ldots$

2.8 Parallel system simulation Method _. . . . . . . . . . . . 15

3 Inverter Controls and Modeling $\quad 17$

3.1 Grid-following inverter model . . . . . . . . . . . . . . . . . . . . . 19

3.2 Fault response of IBR in grid-following mode . . . . . . . . . . . 22

3.3 Impact of grid-following IBRs on the microgrid in grid connected mode 23

3.4 Grid-forming inverter model . . . . . . . . . . . . . . 26

3.5 Grid-forming mode IBRs fault response . . . . . . . . . . . . . . . 29

3.6 Impact of grid-forming IBRs on the microgrid in islanded mode . . . 30

4 Analysis of Existing Protection Devices 35

4.0 .1 Overcurrent Elements _. . . . . . . . . . . . 35

4.0.2 Differential Protection . . . . . . . . . . . . . . 36

4.0.3 Distance Protection . . . . . . . . . . . . . . . . . 36

4.1 Issues faced by the existing protection in the LOHO system $\ldots \ldots 37$

4.1 .1 Bidirectional current . . . . . . . . . . . . . 37

4.1 .2 Low fault current . . . . . . . . . . . . . . . . 40

4.1 .3 Large coordination ranges $\ldots \ldots \ldots \ldots$

4.2 Summary of updates to the protection devices . . . . . . . . . 42 
5 Proposed Protection Method 44

5.1 Example settings calculation . . . . . . . . . . . . . . 47

5.2 Grid-Connected mode coordination \& settings . . . . . . . . . 54

5.3 Islanded mode coordination \& settings $\ldots \ldots \ldots \ldots$

$\begin{array}{lll}6 & \text { Conclusion } & 67\end{array}$

A Appendix A: Code Used to Simulate Faults 69

A.1 Bash Script used to submit batch jobs to the palmetto cluster . . . 69

A.2 Code to generate fault simulation files $\ldots \ldots \ldots \ldots$

B Appendix B: Code to Calculate Device Settings $\quad 80$

B.1 Function to calculate recloser settings . . . . . . . . . 80

B.2 Function to calculate relay settings $\ldots \ldots \ldots \ldots \ldots$ 


\section{List of Figures}

Figure $\quad$ Page

1.1 States with RPS or similar policies [1] . . . . . . . . . . 2

1.2 Projected energy $\operatorname{mix}[2] \ldots \ldots \ldots \ldots \ldots$

1.3 Projected renewables $\operatorname{mix}[2] \ldots \ldots \ldots$. . . . . . . . . . 2

1.4 Possible 100\% IBR microgrid Setup . . . . . . . . . . . . . . . 4

2.1 $12.47 \mathrm{kV}$ utility distribution feeder considered for conversion to a mi-

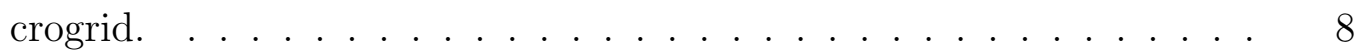

2.2 Three-phase PI line model . . . . . . . . . . . . . . . . . . . . . . . . 9

2.3 Three-phase load model . . . . . . . . . . . . . . . . . . . . 10

$3.1 \quad 12.47 \mathrm{kV}$ utility distribution feeder converted to microgrid with IBRs. 18

3.2 Grid-following IBR connection diagram . . . . . . . . . . . . . . . 19

3.3 Grid-following IBR controller . . . . . . . . . . . . . . . . . 20

3.4 Grid-following IBR PLL \& measurements . . . . . . . . . . . . . . . . 20

3.5 Grid-following IBR current controller . . . . . . . . . . . . . . . . . . 21

3.6 Grid-following IBR VSC reference signal generation . . . . . . . . . . 21

3.7 Fault response of grid following IBR . . . . . . . . . . . . . . 22

3.8 Grid-forming IBR connection diagram . . . . . . . . . . . 26

3.9 Grid-forming IBR controller . . . . . . . . . . . . . . . . . 27

3.10 Grid-forming IBR power, voltage, current calculation . . . . . . . . . 27 
3.11 Grid-forming IBR modulation signal generation . . . . . . . . . . . 28

3.12 Grid-forming IBR fault response . . . . . . . . . . . . . . . . . 30

4.1 LOHO system with bidirectional fault current example . . . . . . . 38

4.2 Example fault current in grid connected mode . . . . . . . . . . . . . 41

4.3 LOHO system with updated protection elements . . . . . . . . . 43

5.1 Settings groups for bidirectional protective devices. . . . . . . . . . 46

5.2 Flow chart of relay trip signal generation . . . . . . . . . . . . 46

5.3 Grid connected $R E C_{F} \mathrm{TCC} \ldots \ldots \ldots$. . . . . . . . . . . . 51

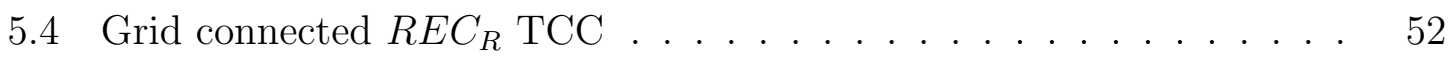

5.5 Islanded $R E C_{F} \mathrm{TCC} \ldots \ldots \ldots \ldots$. . . . . . . . . . . . . 52

5.6 Islanded $R E C_{R} \mathrm{TCC} \ldots \ldots \ldots \ldots$. . . . . . . . . . . 53

5.7 Grid connected zone 2 protection coordination . . . . . . . . . 55

5.8 Grid connected zone 3 protection coordination . . . . . . . . . 56

5.9 Grid connected zone 4 protection coordination . . . . . . . . . . 57

5.10 Grid connected zone 5 protection coordination . . . . . . . . 58

5.11 Islanded mode zone 2 protection coordination . . . . . . . . . 61

5.12 Islanded mode zone 3 protection coordination . . . . . . . . . . . 62

5.13 Islanded mode zone 4 protection coordination . . . . . . . . . 62

5.14 Islanded mode zone 5 protection coordination . . . . . . . . 63 


\section{List of Tables}

Table $\quad$ Page

2.1 LOHO system line parameters . . . . . . . . . . . . . . . 10

2.2 LOHO load ratings . . . . . . . . . . . . . . . . . . 11

2.3 Substation transformer parameters . . . . . . . . . . . . . . 11

2.4 Utility source parameters . . . . . . . . . . . . . . . . . . . . 12

2.5 Base case steady-state line currents . . . . . . . . . . . . . 13

2.6 Base case steady-state load currents . . . . . . . . . . . . . . . 14

2.7 Fault currents without IBR contribution . . . . . . . . . . . . 14

3.1 Line currents and power flow for grid connected microgrid with gridfollowing mode IBRs . . . . . . . . . . . . . . . . . . . . . . 24

3.2 Load currents and power for grid connected microgrid with grid-following mode IBRs . . . . . . . . . . . . . . . . . . . . . . . 24

3.3 Fault currents with grid-following mode IBRs . . . . . . . . . . 25

3.4 Line currents and power flow for islanded microgrid with grid-forming mode IBRs . . . . . . . . . . . . . . . . . . . . . . . . . . 31

3.5 Load currents and power for islanded microgrid with grid-forming mode IBRs . . . . . . . . . . . . . . . . . . . . . 32

3.6 Bus voltages for islanded microgrid with grid-forming mode IBRs . . 33

3.7 Fault currents with grid forming mode IBRs in islanded microgrid state 33 
5.1 Example settings For recloser REC at bus $5 \ldots \ldots$. . . . . . . . 53

5.2 Settings for protective devices in grid-connected mode . . . . . . . . 58

5.3 Operating times for a 3 ph fault during grid-connected operation ${ }^{\text {a, } b} \quad$. $\quad 59$

5.4 Settings for protective devices in islanded mode . . . . . . . . . . . . 64

5.5 Operating times for a 3 ph fault at all buses during islanded operation ${ }^{\mathrm{a}, b} 65$ 


\section{Nomenclature}

DER - Distributed Energy Resources

$I B R \quad$ - Inverter Based Resources

RPS - Renewable Portfolio Standards

LOHO - Test Feeder name

PCC - Point of Common Coupling

$O C \quad-\quad$ Overcurrent

UV - Undervoltage

3PHF - 3-phase fault

LLF - Line-Line fault

SLGF - Single line to ground fault

VSC - Voltage Source Converter

$U_{\text {ref }} \quad-\quad$ Reference signal for VSC converter

TCC - Time Current Curves

$R n_{F} \quad$ - Relay "n" forward direction setting

$R n_{R} \quad$ - Relay "n" reverse direction setting

Ot $\quad$ - device operating time

$T D S \quad$ - Time Dial Setting

MOC - Minimum Operating current / Pickup current 


\section{Chapter 1}

\section{Introduction and Literature}

\section{Review}

There has been a significant push in many parts of the world to start using more renewable energy resources due to rising concerns regarding the environmental effects of fossil-based energy production and the decreasing cost of renewable energy sources. More than half the states in the United States (US) have enacted legislation to increase the share of renewables in their energy mix; for example, Washington DC, California, and Hawaii have increased their Renewable Portfolio Standards (RPS) target to $100 \%$ renewable before 2045 . Figure 1.1 shows the states with RPS targets or similar regulations in the United States. Many of the RPS targets also include specific requirements for solar PV based generation to ensure variety in their energy generation [1].

In the US and around the world renewable energy generation is expected to increase over the next few decades, with IBRs such as solar and wind generation, in particular, driving the conversion from traditional fossil fuel-based sources [2]. Figures 1.2 and 


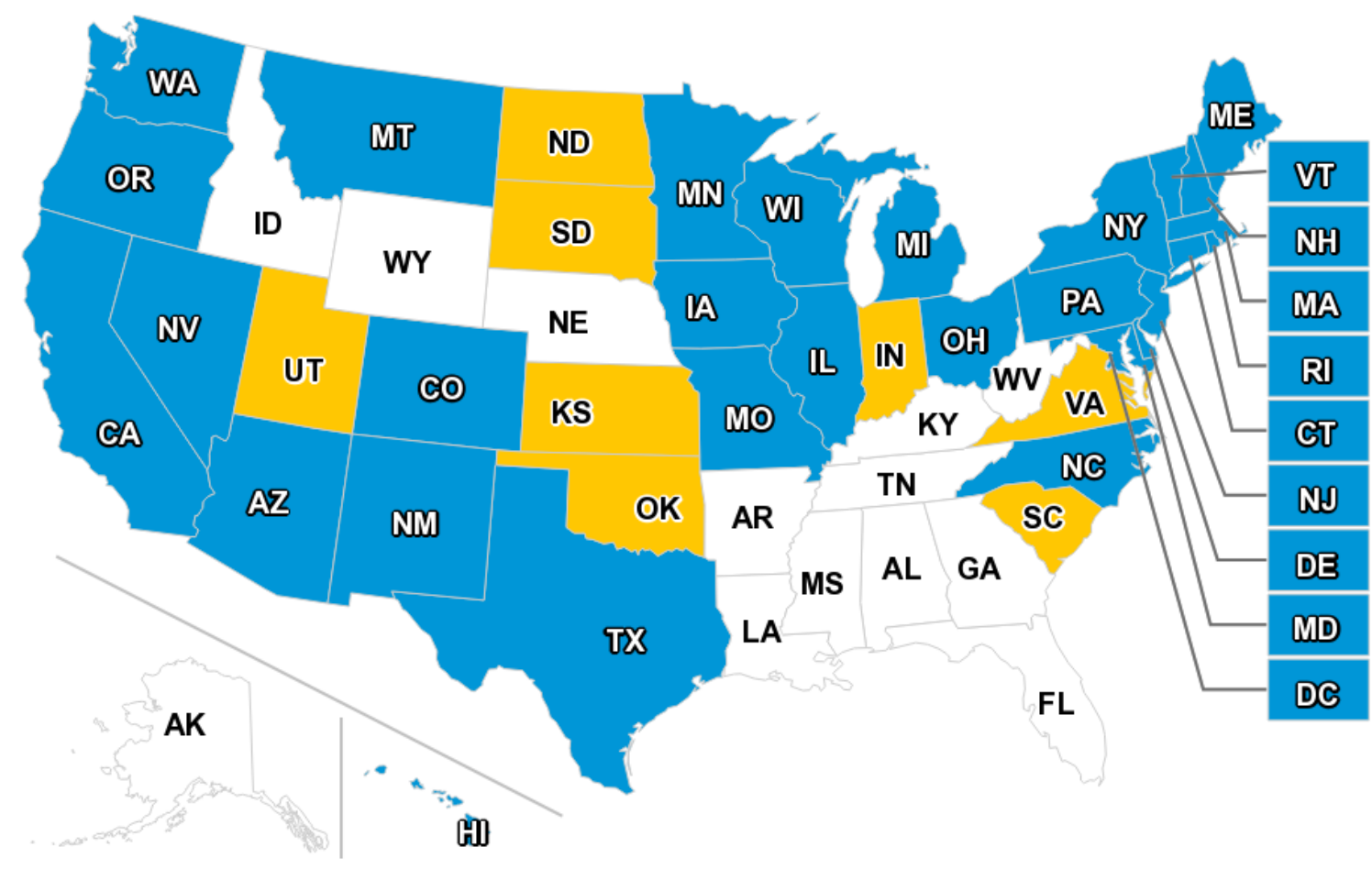

\section{Has renewable portfolio standards Has renewable portfolio goals}

Figure 1.1: States with RPS or similar policies [1]

1.3 show the projected growth of renewables in the US. As power grids around the world continue to integrate more renewable energy resources. The increase in demand for more sustainable, reliable, and resilient power has led to a subset of the power grid known as a microgrid, powered by renewable energy sources.

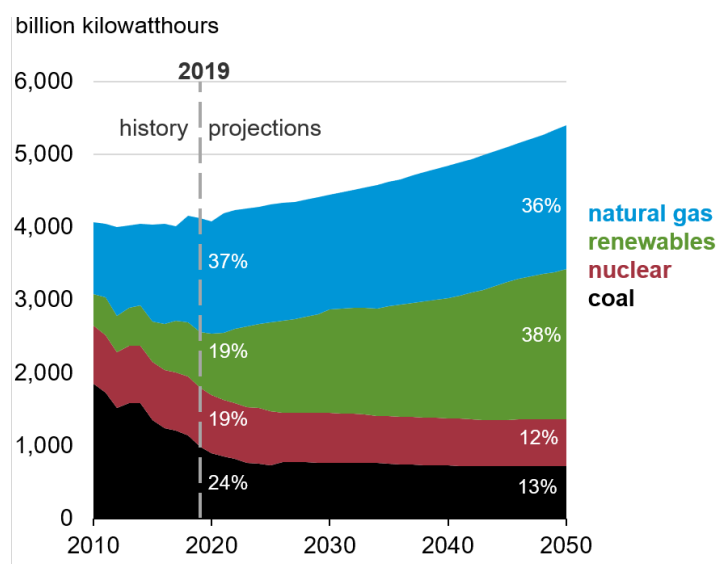

Figure 1.2: Projected energy mix [2]

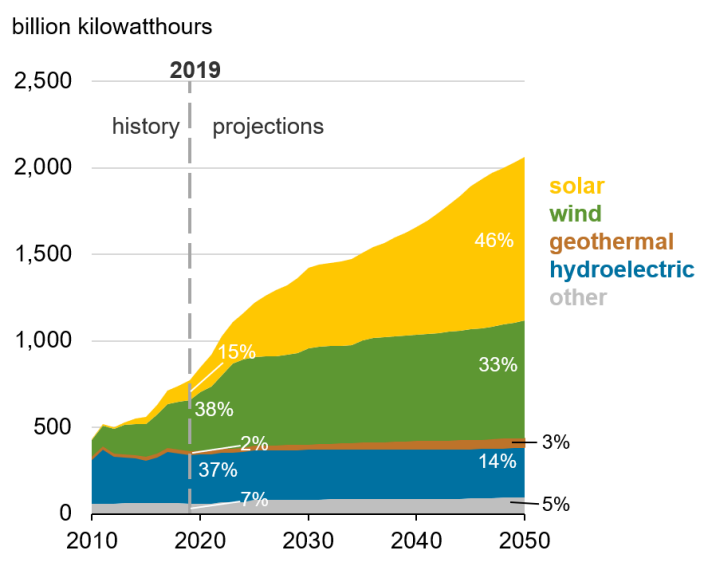

Figure 1.3: Projected renewables mix [2] 


\subsection{Inverter based resources and microgrids}

As renewables continue to replace or augment traditional fossil-fuel-based resources on the eclectic grid, a larger percent of the grid's energy mix will be inverter-based resources (IBRs) such as solar, wind, and battery energy storage systems (BESS). IBRs are sources that are interfaced with the electric grid through an inverter to convert and regulate the energy generated by renewable sources such as solar and wind. IBRs unlike traditional generation methods, are typically located close to loads and often connected at the distribution level. These IBRs and neighboring loads on a feeder can be grouped together into a subnetwork known as a microgrid.

Microgrids are a combination of generation resources and load, forming an electrically sustainable grid that can function either connected to the larger grid or in an islanded mode. An example of $100 \%$ inverter feed microgrid is shown in figure 1.4. As renewables gain popularity and distributed generation becomes more commonplace, it is expected that microgrids will be created by adding Distributed Energy Resources (DERs) at the distribution level and converting existing feeders to microgrids. In grid-connected mode, a microgrid shares its load and generation resources, whereas in islanded mode it is disconnected from the utility grid and is self-sufficient. Distribution level microgrids tend to have a significant amount of their energy generation from solar PV and/or storage, which connect through inverters. When multiple IBRs

connect to microgrids, their control in grid-connected and islanded modes need to be carefully designed for smooth operation and transition between modes. Due to the distinctly different fault response of IBRs, which includes limiting the positive-sequence current to values comparable to its rated output and blocking of negative-sequence and zero-sequence currents, the protection of microgrids also needs careful attention. 


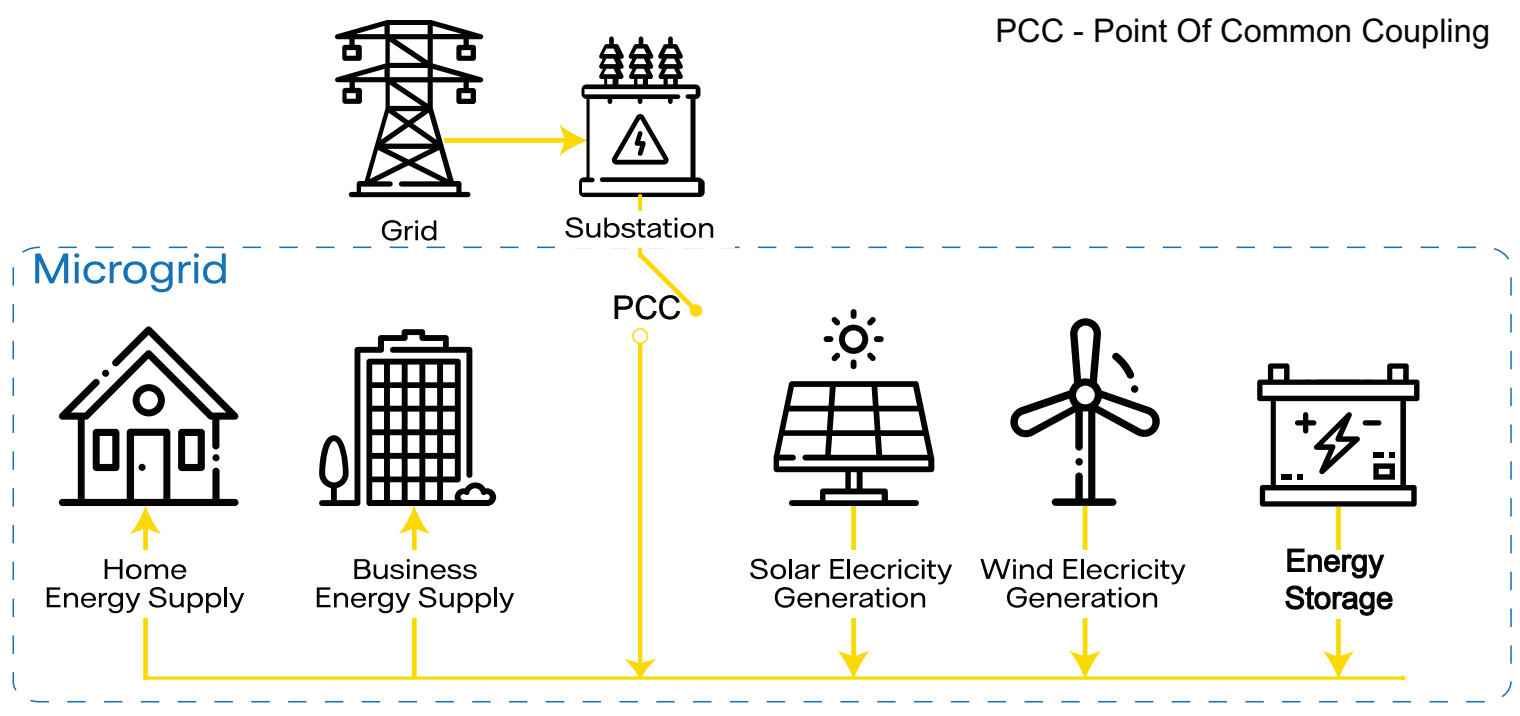

Figure 1.4: Possible 100\% IBR microgrid Setup

\subsection{Microgrid protection literature review}

A wide array of protection schemes have been proposed for microgrids. Each assumes a certain topology, source-type, and operating conditions [3]. A summary of such schemes is provided in [4]. The schemes are based on overcurrent, undervoltage, a combination of the two, distance, differential, harmonic-content, and traveling waves, many using various communication infrastructure. Shiles et al. [5] provide an overview of the protection methods used in various microgrid projects across North America. Several principles and methods are mentioned based on undervoltage, voltage-restrain, impedance, differential, directional overcurrent, and adaptive, some even including fault current limiters. It underscores that the protection methods are highly dependent on the type of sources used in the system, and the system topology. None of the methods discussed has selective protection for an islanded microgrid. A protection scheme for micro-grids which depends primarily on undervoltage relays for islanded operation and overcurrent relays for grid-connected operation is proposed in [6]. A key aspect of the protection proposed in [6] is that it does not require communication or adaptive protective devices; however, the proposed method is for 
low voltage $(120 \mathrm{~V})$ microgrids and undervoltage coordination can be very difficult to achieve. Laaksonen et al. [7] present an adaptive protection and control scheme using communication, developed for the Hailuoto Island microgrid, where the relay settings are modified depending on the mode of operation. Sources, in this case, consist of diesel generators and wind turbines. The literature survey reveals that protection schemes suitable for realistic microgrids with 100\% IBRs have not been sufficiently explored. Brahma et al. $[8,9]$ provides some insight into protection of islanded, unbalanced microgrids with $100 \%$ IBRs, but does not model a test system with multiple inverters to illustrate the control and protection aspects.

Overall while many methods have been proposed in literature, few of the papers explore the case of a $100 \%$ inverter-based realistic microgrids with multiple inverterbased resources on a realistic system. A microgrid's ability to switch from gridconnected to islanded mode of operation poses several challenges from a protection perspective ranging for fault detection, selectivity, security, and device coordination. Inverter based sources pose an additional challenge due to the limit on the amount of current supplied during a fault to protect their power electronics components [10]. The limited current poses challenges as it drastically reduces the amount of fault current observed by traditional protective devices, which heavily rely on current magnitude for fault detection, creating difficulties in identifying and isolating faults in the microgrid. Due to low fault currents, most of the conventional protection principles fail to provide reliable protection [11]. The low fault current during islanded mode compared to gridconnected mode also pose challenges in terms of protective device coordination, as the coordination ranges grow large due to the low fault current during islanded mode and high fault current during grid-connected mode. In many cases depending on the microgrid composition, a singular protection scheme for both grid-connected and islanded modes may not be possible. 


\subsection{Goals}

The primary goal of this project is to analyze the protection of a real-life distribution system being converted to a microgrid. In order to do this, the real-world LOHO feeder located in the southwestern US needs to be modeled in MATLAB Simulink. The model can then be converted to a microgrid by connecting three solar PV units of varying capacity. The inverter models used to convert the feeder to a microgrid will mimic the dynamics of commercially available inverters tested at Sandia National Laboratories (SNL). Once the inverters are modeled and connected to the feeder, the existing protection scheme of the feeder can be analyzed in the presence of the inverters in grid-connected and islanded modes. This analysis will highlight how and why the existing protection scheme will not work in the proposed microgrid. Finally, based on the analysis an adaptive protection scheme will be created, including selective protection for the island, using protective devices available in the market today. 


\section{Chapter 2}

\section{LOHO Test System \& Modeling}

\subsection{System overview}

The real-world $12.47 k V$, 15-bus distribution feeder used for this study, referenced henceforth as LOHO is shown in figure 2.1. It is proposed to be converted into a microgrid by adding PV generation of $258 \mathrm{~kW}, 1 \mathrm{MW}, 10 \mathrm{MW}$ at buses 15,12 and 14 respectively. This feeder has a total load of approximately 3 MVA, so in gridconnected mode, the microgrid will feed its load and feed power back to the utility system, all inverters operating at unity power factor. In islanded mode, it will curtail its real power output and supply the required real and reactive power to the microgrid, while maintaining voltage and frequency at acceptable values. Inverters at buses 12 and 14 connect to the system through $480 \mathrm{~V} / 12.47 \mathrm{kV}, Y \mathrm{G} / \mathrm{YG}$ transformers, whereas the IBR at bus 15 connects at $480 \mathrm{~V}$ through a $Y G / Y G$ transformer. The system is modeled in MATLAB/Simulink using Simscape power systems library. The grid is molded as a three-phase $115 \mathrm{kV}$ source connected through a $115 \mathrm{kV} / 12.47 \mathrm{kV}$ $\triangle / Y G$ substation transformer rated at $30 M V A$. The lines are molded as PI sections. The loads are modeled as wye-connected constant impedances. 


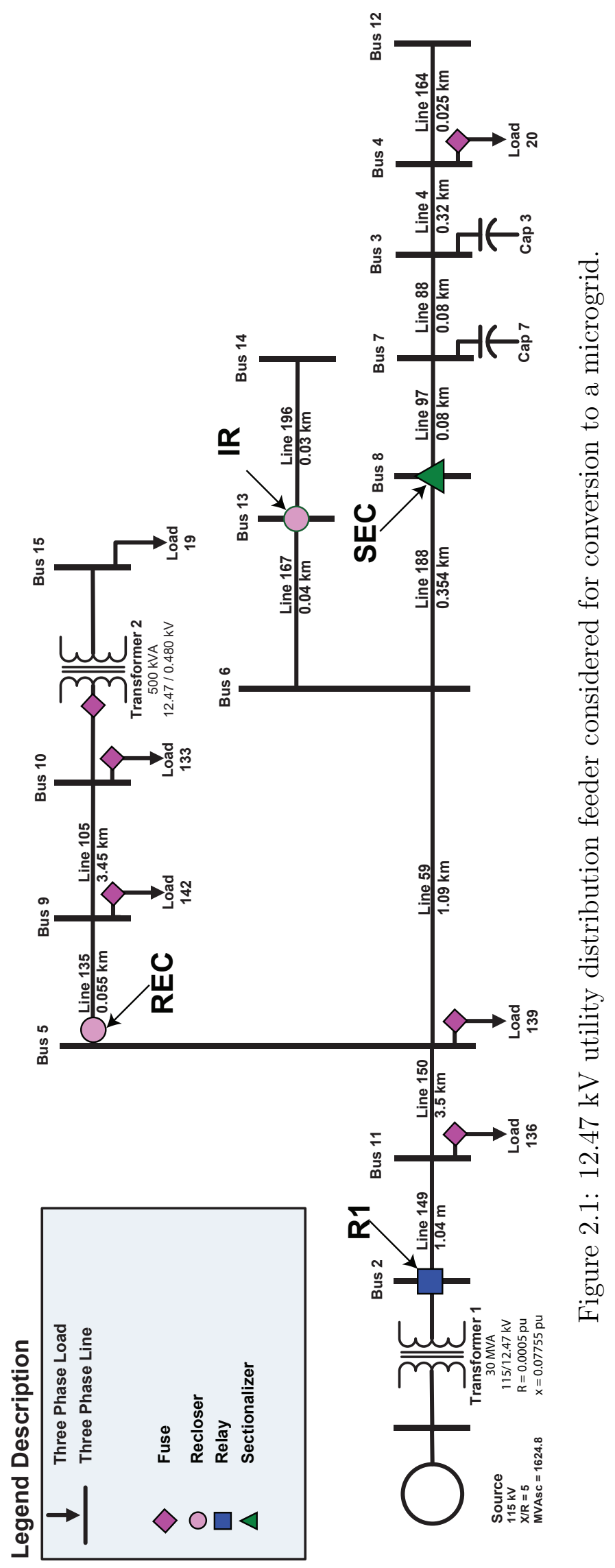




\section{$2.2 \quad$ Lines}

The system is a reduced model of a real feeder which consists of 12 distribution lines. The lines are modeled as three-phase PI section lines. The individual line parameters are shown in table 2.1 except the positive and zero sequence line shunt capacitance $(C 1$ and $C 0)$ which were set to $1.12 E-7$ for all lines. The three-phase PI section line" available in Simulink implements a balanced three-phase transmission line model with parameters lumped in a PI section. While the balanced lines don't capture all the detail of the unbalanced distribution feeder, this approximation was made due to the limitation of software. A diagram of the three-phase PI section line is shown in figure 2.2. Line parameters are calculated based on equation 2.1.

$$
\begin{aligned}
R_{s} & =\frac{\left(2 R_{1}+R_{0}\right)}{3} \\
L_{s} & =\frac{\left(2 L_{1}+L_{0}\right)}{3} \\
R_{m} & =\frac{\left(R_{0}-R_{1}\right)}{3} \\
L_{m} & =\frac{\left(L 0-L_{1}\right)}{3} \\
C_{g} & =\frac{3 C_{1} C_{0}}{\left(C_{1}-C_{0}\right)}
\end{aligned}
$$

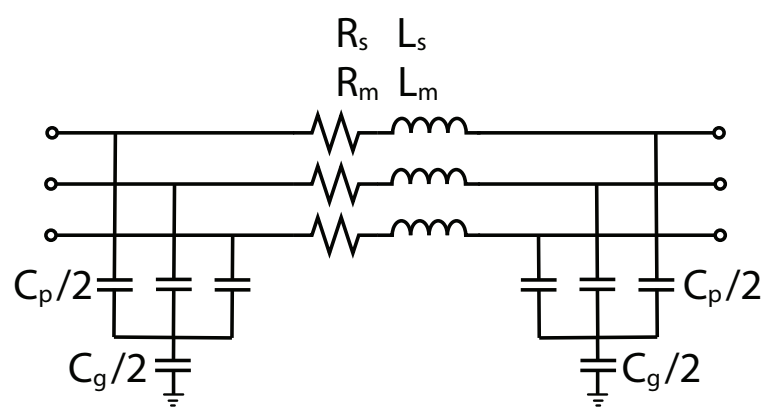

Figure 2.2: Three-phase PI line model 


\begin{tabular}{|c|c|c|c|c|c|}
\hline Name & Length $(\mathrm{km})$ & $\mathrm{R} 1(\Omega / \mathrm{km})$ & $\mathrm{R} 0(\Omega / \mathrm{km})$ & $\mathrm{L} 1(\mathrm{H} / \mathrm{km})$ & $\mathrm{L} 0(\mathrm{H} / \mathrm{km})$ \\
\hline L149 & 0.001 & 0.0803806 & 0.2004593 & 0.0002976 & 0.0001758 \\
\hline L150 & 3.57 & 0.0768443 & 0.3632861 & 0.0009390 & 0.0032733 \\
\hline L135 & 0.05 & 0.4373360 & 0.8034780 & 0.0013541 & 0.0042251 \\
\hline L105 & 3.45 & 0.7137992 & 1.0845650 & 0.0013745 & 0.0042357 \\
\hline L59 & 1.09 & 0.0744751 & 0.3484252 & 0.0009825 & 0.0034924 \\
\hline L188 & 0.35 & 0.0744751 & 0.3484252 & 0.0009825 & 0.0034924 \\
\hline L97 & 0.08 & 0.0744751 & 0.3484252 & 0.0009825 & 0.0034924 \\
\hline L88 & 0.08 & 0.0744751 & 0.3484252 & 0.0009825 & 0.0034924 \\
\hline L4 & 0.32 & 0.0815039 & 0.3488770 & 0.0009068 & 0.0030888 \\
\hline L164 & 0.03 & 0.1049869 & 0.3267717 & 0.0003150 & 0.0002237 \\
\hline L167 & 0.04 & 0.1463255 & 0.4202756 & 0.0010548 & 0.0035646 \\
\hline L196 & 0.03 & 0.1820739 & 0.3053387 & 0.0005815 & 0.0005143 \\
\hline
\end{tabular}

Table 2.1: LOHO system line parameters

\subsection{Loads}

The system consists of 6 loads which are used to represent a variety of loads on the actual feeder. Additionally, 2 capacitor banks are also included. Table 2.2 shows the individual load parameters. The loads are modeled using the series RLC load for each phase creating an unbalanced wye-connected constant impedance load. The load impedance is determined using the nominal voltage $(\mathrm{V})$, active power $(\mathrm{P})$, and reactive power $(\mathrm{Q})$ specified using the equation 2.2. Figure 2.3 shows the series RLC Load model.

$$
Z=R+j X=\frac{V^{2}}{P}+j \frac{V^{2}}{Q}
$$

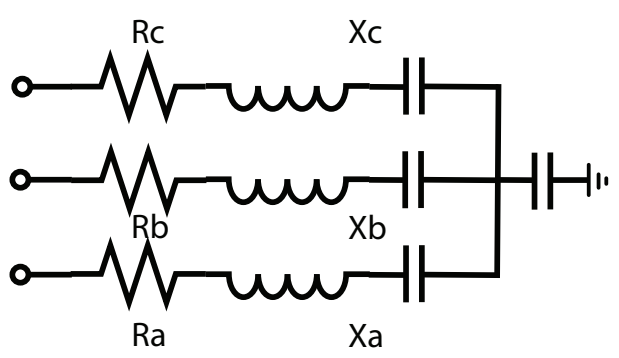

Figure 2.3: Three-phase load model 


\begin{tabular}{|c|c|c|c|c|c|c|}
\hline Load & Pa $(\mathrm{kW})$ & Qa $(\mathrm{kVAR})$ & $\mathrm{Pb}(\mathrm{kW})$ & Qb $(\mathrm{kVAR})$ & $\mathrm{Pc}(\mathrm{kW})$ & Qc $(\mathrm{kVAR})$ \\
\hline 136 & 0 & 0.003 & 3.860 & 0.489 & 2.670 & 0.302 \\
\hline 139 & 0.028 & -0.009 & 2.288 & 0.413 & 0.394 & 0.154 \\
\hline 142 & 97.118 & 22.060 & 100.579 & 22.407 & 116.317 & 25.615 \\
\hline 133 & 107.384 & 11.558 & 108.561 & 11.911 & 118.930 & 12.605 \\
\hline 19 & 27.700 & 16.900 & 27.700 & 16.900 & 27.700 & 16.900 \\
\hline 20 & 628.333 & 430.666 & 628.333 & 430.666 & 628.333 & 430.666 \\
\hline
\end{tabular}

Table 2.2: LOHO load ratings

\subsection{Transformers}

The system contains a substation transformer and a distribution transformer, along with the two interfacing transformers to be added to connect the PV systems to the microgrid. All transformers are modeled as three single-phase transformers using the Simscape "Three-Phase Transformer (Two Windings)" transformer. The parameters of the transformers are shown in table 2.3.

\begin{tabular}{|c|c|c|c|c|}
\hline Transformer Name & Transformer1 & Transformer2 & $T_{\text {Inv1 }}$ & $T_{\text {Inv2 }}$ \\
\hline winding 1 connection & $\Delta$ & Yg & Yg & Yg \\
\hline winding 2 connection & Yg & Yg & Yg & Yg \\
\hline Nominal Power (kVA) & 30000 & 1000 & 1500 & 5000 \\
\hline Winding 1 Voltage (kV) & 115 & 12.47 & 12.47 & 12.47 \\
\hline Winding 2 Voltage (kV) & 12.47 & 0.480 & 0.480 & 0.480 \\
\hline Winding 1 Resistance (pu) & 0.00025 & 0.002 & 0.005 & 0.005 \\
\hline winding 2 Resistance (pu) & 0.00025 & 0.002 & 0.005 & 0.005 \\
\hline winding 1 Inductance (pu) & 0.07755 & 0.035 & 0.01 & 0.01 \\
\hline winding 2 Inductance (pu) & 0.07755 & 0.035 & 0.01 & 0.01 \\
\hline Magnetization resistance (pu) & 500 & 500 & 500 & 500 \\
\hline Magnetization inductance (pu) & 500 & 500 & 500 & 500 \\
\hline Bus1 name & Bus1 & Bus10 & Bus12 & Bus14 \\
\hline Bus2 name & Bus2 & Bus15 & Inv2 & Inv3 \\
\hline
\end{tabular}

Table 2.3: Substation transformer parameters 


\subsection{Grid source}

The utility grid is modeled as a swing generator to emulate the grid's capabilities to supply and absorb a large amount of power when needed. The swing generator is also configured to match the utility fault current characteristics. The generator is modeled using the Simscape electrical "Three-Phase Source" model with the parameters shown in table 2.4 .

\begin{tabular}{|c|c|}
\hline Configuration & Yg \\
\hline Base voltage $(\mathrm{kV})$ & 115 \\
\hline Phase A angle $\left(^{\circ}\right)$ & 0 \\
\hline Frequency $(\mathrm{Hz})$ & 60 \\
\hline Source resistance $(\Omega)$ & 1.5 \\
\hline Source inductance $(\mathrm{H})$ & 0.02 \\
\hline 3-Phase $M V A_{s c}(\mathrm{MVA})$ & 1624.8 \\
\hline $\mathrm{X} / \mathrm{R}$ Ratio & 5 \\
\hline
\end{tabular}

Table 2.4: Utility source parameters

\subsection{Existing protection elements in the system}

The LOHO system has several typical distribution protection devices in the system. The feeder's main protection device is an overcurrent (OC) relay located at bus2 labeled "R1" in figure 2.1. This relay serves as the ultimate backup protection which coordinates with the devices downstream. A recloser is located at bus 5 labeled "REC" in figure 2.1. This recloser acts as the primary protection for buses 9, 10 and 15. The recloser is coordinated with the Relay R1 which operates as the backup if recloser REC fails to operate. The system also contains a sectionilizer located at bus 8 labeled as "SEC" in figure 2.1. The sectionalizer works in conjunction with the relay R1's re-closing function to count. The sectionilizer acts as the primary protection for buses 7, 3, 4 and 12 with once again the relay R1 acting as a backup 
if the sectionilizer fails to lockout. The system is also protected by a number of load fuses used to isolate loads from the feeder if a fault occurs in the loads. These load fuses are coordinated with the recloser whenever possible, however, many of the load fuses are not coordinated with upstream protection devices.

\subsection{Base case steady-state \& fault simulations}

The LOHO system was simulated without the addition of the PV systems to set a base case for comparison to the microgrid cases. This case also represents the system when the PV is unavailable during a period of low irradiance or night. The system line currents and load currents are shown in table 2.5 and table 2.6, respectively. The system draws roughly $122 \mathrm{~A}$ of current on each phase with $1 \%$ unbalance at the low voltage side of the substation transformer.

\begin{tabular}{|c|l|l|l|}
\hline Line \# & IA (A) & IB (A) & IC (A) \\
\hline T1 (HV) & 13.45 & 13.53 & 13.5 \\
\hline 149 & 120.8 & 122.1 & 123.1 \\
\hline 150 & 120.6 & 121.5 & 122.6 \\
\hline 135 & 32.65 & 33.28 & 34.26 \\
\hline 105 & 18.84 & 19 & 17.79 \\
\hline T2 (HV) & 4.5 & 4.5 & 1.55 \\
\hline T2(LV) & 115.3 & 115.3 & 38.69 \\
\hline 59 & 90.36 & 90.3 & 90.37 \\
\hline 167 & \multicolumn{4}{|l|}{ N/A PV not connected } \\
\hline 196 & \multicolumn{4}{|l|}{ N/A PV not connected } \\
\hline 188 & 90.27 & 90.21 & 90.28 \\
\hline 97 & 90.24 & 90.18 & 90.25 \\
\hline 88 & \multicolumn{4}{|l|}{105.6} & 105.5 & 105.6 \\
\hline 4 & 105.6 & 105.6 & 105.6 \\
\hline 164 & \multicolumn{3}{|l}{ N/A PV not connected } \\
\hline
\end{tabular}

Table 2.5: Base case steady-state line currents

In order to measure the fault currents supplied by the grid when IBRs are not available 


\begin{tabular}{|c|l|l|l|}
\hline Load \# & IA (A) & IB (A) & IC (A) \\
\hline 136 & 0.001 & 0.54 & 0.37 \\
\hline 139 & 0.004 & 0.32 & 0.06 \\
\hline 142 & 13.83 & 14.30 & 16.54 \\
\hline 133 & 14.89 & 15.04 & 16.51 \\
\hline 19 & 115.30 & 115.20 & 38.68 \\
\hline cap7 & 83.37 & 83.32 & 83.38 \\
\hline cap3 & \multicolumn{3}{|l|}{ Not connected } \\
\hline 20 & \multicolumn{3}{|l|}{} \\
\hline
\end{tabular}

Table 2.6: Base case steady-state load currents

faults were simulated on each bus of the system. A 3ph fault, LL fault on phase A-B and SLG fault on phase A were simulate the fault currents supplied by the grid are shown in table 2.7. The maximum fault current of $8292 \mathrm{~A}$ is observed at bus 2 as expected. The lowest fault current of $234 \mathrm{~A}$ was observed on bus 15 which is the furthest point on the feeder from the substation and is behind a transformer. Overall it can be seen that the grid provides a large amount of fault current compared to the load current in almost all cases, exceeding the load current by a factor of 20 .

\begin{tabular}{|l|l|l|l|}
\hline Bus \# & 3phGF (A) & LLF (A) & SLGF (A) \\
\hline 2 & 8011 & 6943 & 8292 \\
\hline 11 & 8010 & 6941 & 8291 \\
\hline 5 & 3296 & 2855 & 2240 \\
\hline 9 & 3249 & 2814 & 2204 \\
\hline 10 & 1478 & 1280 & 1003 \\
\hline $15(\mathrm{LV})$ & 7121 & 6152 & 6086 \\
\hline $15(\mathrm{HV})$ & 274 & 237 & 234 \\
\hline 6 & 2770 & 2400 & 1808 \\
\hline 13 & 2753 & 2384 & 1795 \\
\hline 14 & 2745 & 2378 & 1791 \\
\hline 8 & 2634 & 2282 & 1702 \\
\hline 7 & 2605 & 2257 & 1680 \\
\hline 3 & 2578 & 2233 & 1660 \\
\hline 4 & 2483 & 2151 & 1594 \\
\hline 12 & 2480 & 2148 & 1593 \\
\hline
\end{tabular}

Table 2.7: Fault currents without IBR contribution 


\subsection{Parallel system simulation Method}

Once the system was modeled in MATLAB/Simulink the system needed to be run repeatedly to test the various scenarios ranging from steady-state cases for each microgrid operating mode to fault simulation on each bus in each microgrid mode. These simulations can be computationally intensive and require a large amount of computation capability to complete in a reasonable time frame. Additionally, these simulations produce a considerable amount of data as the voltage and currents seen by each protective device and IBR needed to be recorded for later use in the protection coordination. On a personal laptop computer with 6 cores and 12 threads operating at $4.1 \mathrm{GHz}, 16 \mathrm{~GB}$ of ram, and an NVMe SSD for rapid storage each 15-second fault simulation with a time step of $1 \mu$ s took roughly 45 minutes to complete producing a 1GB file for each simulation. $1 \mu$ s time step was needed to simulate the short lines and for the detailed modeling of inverters.

To perform a complete fault analysis in each microgrid operating mode at a minimum 90 simulations needed to be carried out, 45 faults per simulation mode, 3 faults $(3 \mathrm{phF}$, LLF, SLGF) at 15 buses. If these were performed on a single computer these would require $100 \mathrm{~GB}$ of local storage, and more importantly roughly 70 hours of simulation time alone with additional time to analyze and process the output for each variation of the model, i.e. different fault resistances, modified IBR parameters, etc. Therefore to reduce the time for the fault analysis the fault simulations were carried out in parallel using Clemson University's Palmetto cluster computing system. The fault simulations were carried out using the palmetto batch job system (PBS) using a bash script provided in Appendix A.1. The script runs all 16 simulations simultaneously, 15 fault simulation, one at each bus, and a steady-state case. Multiple jobs can be

submitted at once; therefore 3 jobs, one for each fault type can be submitted leading to all faults being simulated in parallel. On the palmetto cluster computer, the 
simulation jobs typically took around 30 minutes to complete all simulations. This greatly reduces the amount of time spent on simulation and fault data generation.

The bash script takes Simulink model files (.slx) as inputs and simulates the models in parallel based on the setting in the bash script. The simulation models for different fault types and resistances were auto-generated from as single model file, in which the fault block was placed and connected programmatically, and the setting set according to the selected inputs. The MATLAB code to generate the fault simulation models used to simulate faults and perform fault analysis can be found in Appendix A.2. 


\section{Chapter 3}

\section{Inverter Controls and Modeling}

In order to convert the feeder described in Chapter 2 to a microgrid, distributed renewable generation is added to the system. Three inverters based resources are added at buses $15,12,14$ with a capacity of $258 \mathrm{~kW}, 1 \mathrm{MW}, 10 \mathrm{MW}$ respectively. These IBRs need to be capable of operating in grid-forming and grid-following control modes. During the microgrids grid-connected mode, the inverters will operate in grid-following mode providing the set-point real power to the microgrid, while the system reactive power demand is supplied by the utility grid. During the islanded mode, the inverters operate in grid-forming mode, where they behave like the grid-source, providing voltage and frequency regulation. Therefore two types of control modes are designed for inverter models a grid-following mode to be used during grid-connected microgrid operation, and grid-forming, to be used in islanded mode. Figure 3.1 shows the updated feeder converted to microgrid with the addition of the IBRs. 


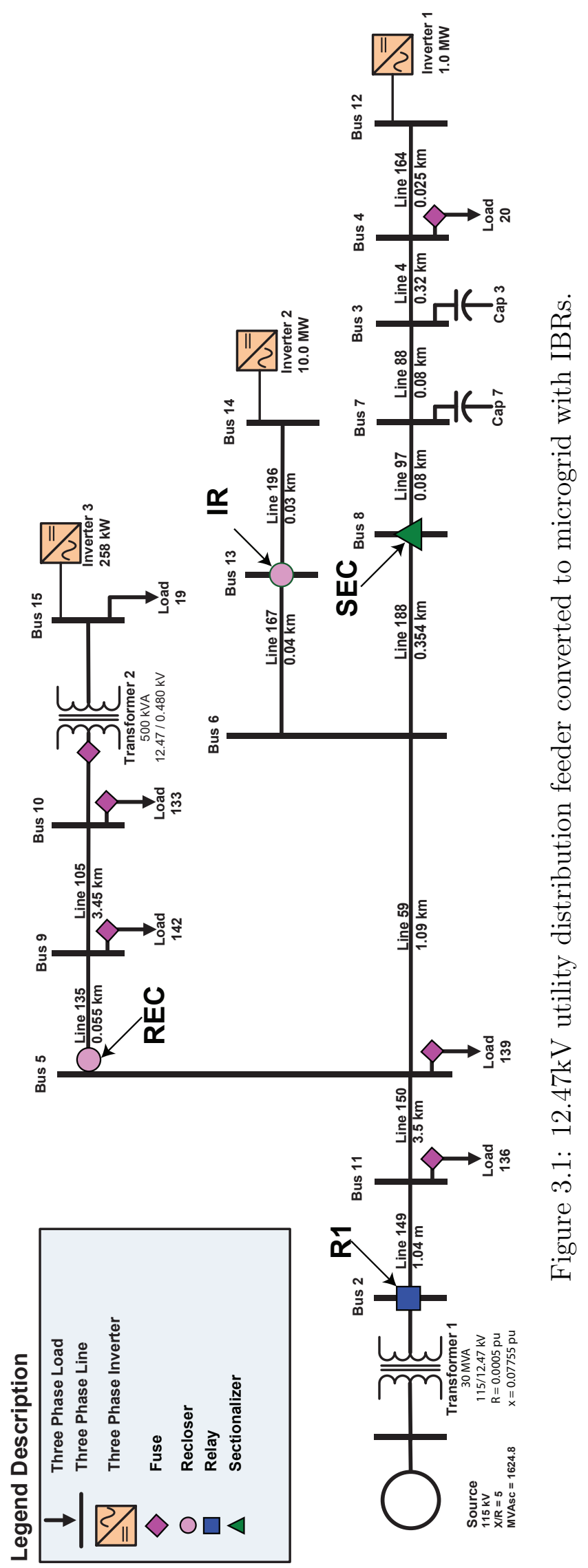




\subsection{Grid-following inverter model}

During the grid-connected mode, the inverters operate in grid-following mode providing the rated or set-point real power to the microgrid, while reactive power is supplied by the utility grid. The grid-following mode inverter-models are based on [12] and [13] as average models using MATLAB's Simscape Electrical Specialized Power Systems library. The overview of the grid-following mode IBR connection and setup is shown in figure 3.2. The grid following mode inverter uses a synchronous reference frame phase-locked loop (PLL) to track the voltage and frequency at the inverter's point of common coupling (PCC). The measured voltage is used as the reference by the PLL to synchronize the inverter PI controllers that regulate the injection of real and reactive power at the $\mathrm{PCC}$. The grid following mode controller is shown in figure 3.3.

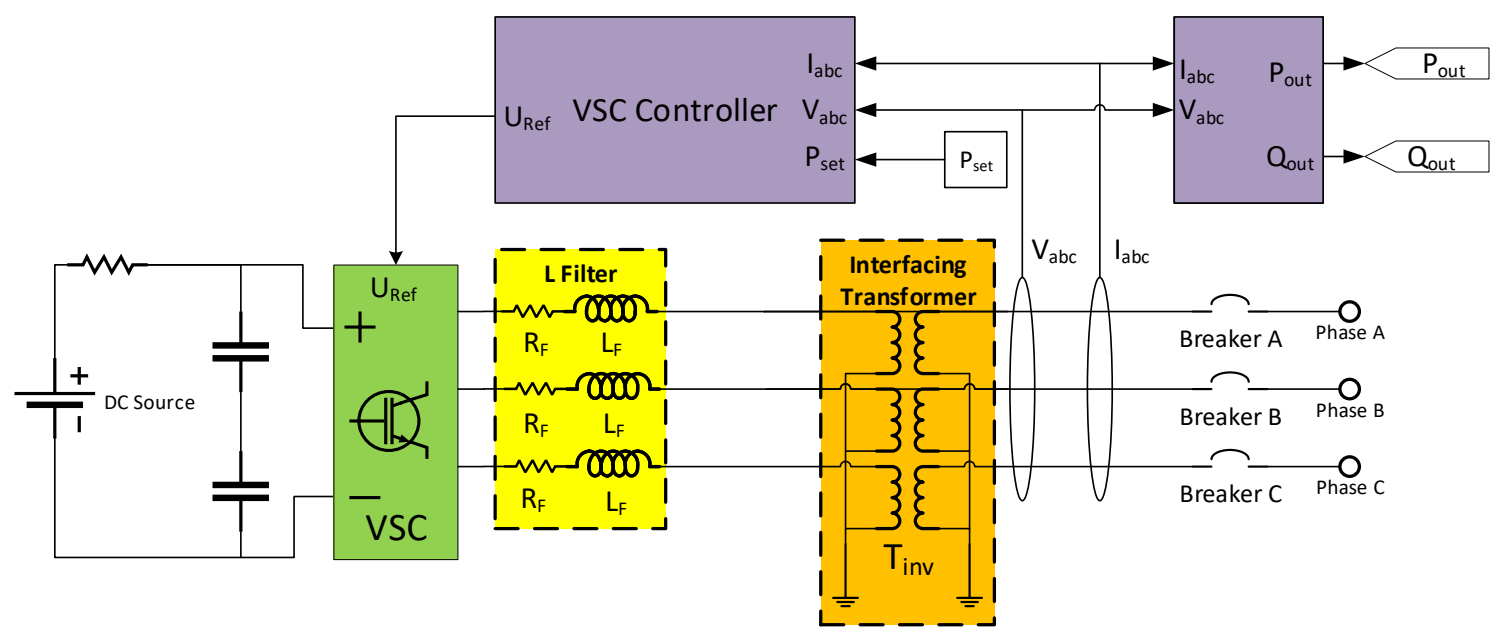

Figure 3.2: Grid-following IBR connection diagram

The controller converts the measured output voltage (Vabc) and current (Iabc) signals to the $d q$ reference frame utilizing a PLL and reference frame conversation as shown in figure 3.4 to calculate $V_{d}, V_{q}, I_{d}, I_{q}$ and $\omega t$. The calculated voltages and current values along side the reference currents $I_{d}^{*}$ and $I_{q}^{*}$ are used by the current controller shown in figure 3.5 to generate the voltage references $V_{d}^{*}$ and $V_{q}^{*}$ using the equations 


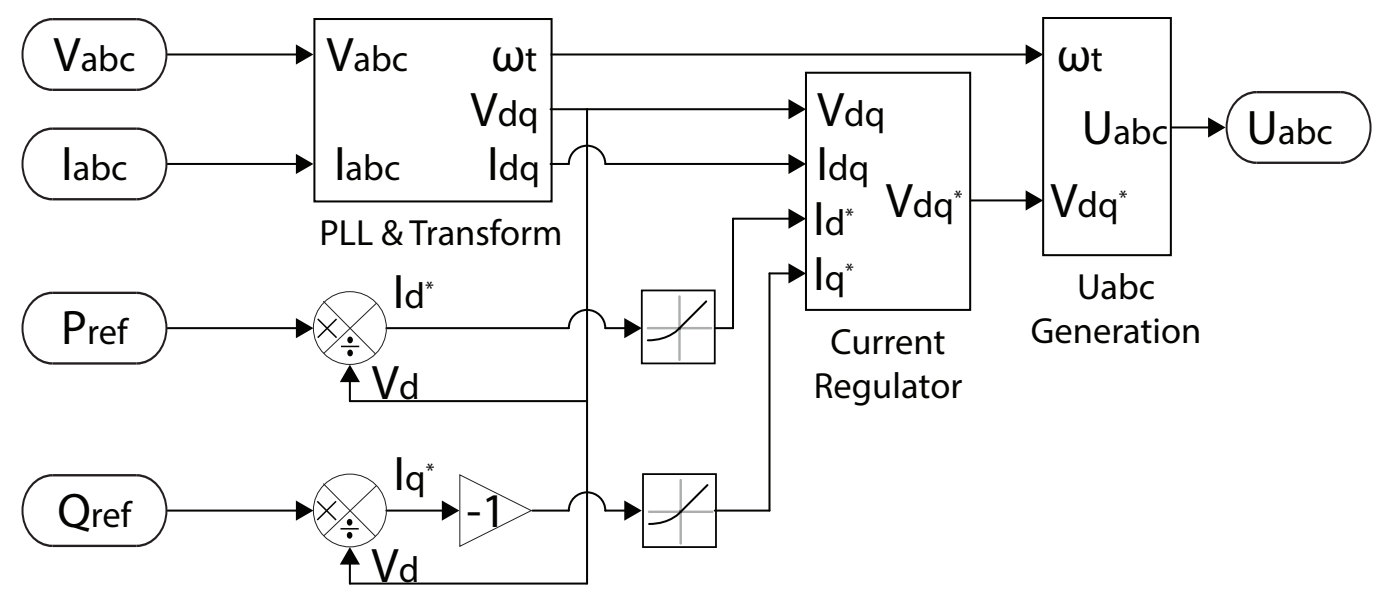

Figure 3.3: Grid-following IBR controller

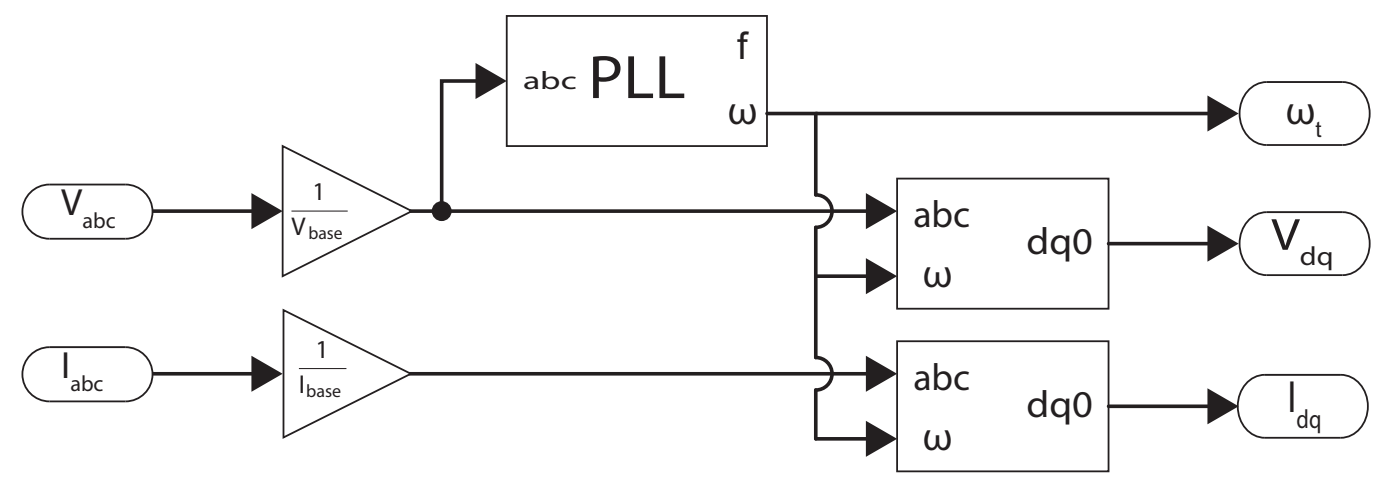

Figure 3.4: Grid-following IBR PLL \& measurements

listed in equation 3.1 .

$$
\begin{aligned}
& V_{d}^{*}=V_{d}+I_{d} * R_{t o t}-I_{q} * L_{t o t}+P I\left(I_{d}^{*}-I_{d}\right) \\
& V_{q}^{*}=V_{q}+I_{q} * R_{t o t}-I_{d} * L_{t o t}+P I\left(I_{q}^{*}-I_{q}\right)
\end{aligned}
$$

During grid-connected operation, $I_{q}^{*}$ is typically set to 0 to achieve unity power factor in grid-following mode. The current control loop is based on a $d q$ current-controlled scheme with feed-forward compensation for both, the grid voltage, and the $i_{d}$ and $i_{q}$ cross-coupling terms that are intrinsic to the analysis of the system in the $d q$-frame [14]. The current controller also implements a current-limiting feature with a simple saturation limit on the reference current generated by the current controller, limiting the current to 2 per unit (pu), based on the inverter rating. This limit is consistent 


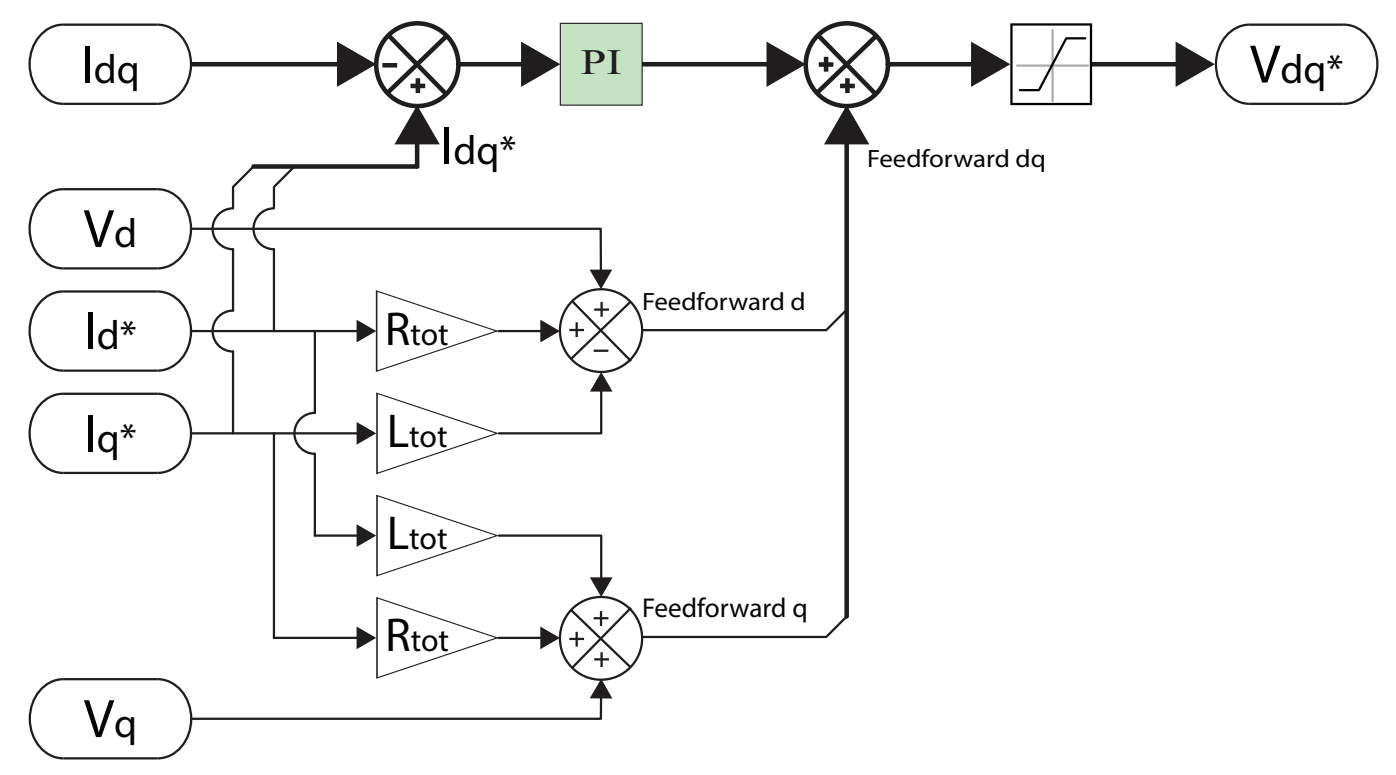

Figure 3.5: Grid-following IBR current controller

with inverters tested at SNL [10]. The reactive support during low voltage ride through (LVRT) is also incorporated as described in [12]. Once the reference converter voltages $V_{d}^{*}$ and $V_{q}^{*}$ is calculated by the current controller they are then converted to the signal that drives the average model voltage source converter as shown in figure 3.6. The reference generation converts the $d q$ reference frame voltage to the 3 phase reference signal $\left(U_{a b c}^{*}\right)$ used to drive the VSC which outputs the commanded voltage and current to the grid through an inductive filter and an interconnection transformer.

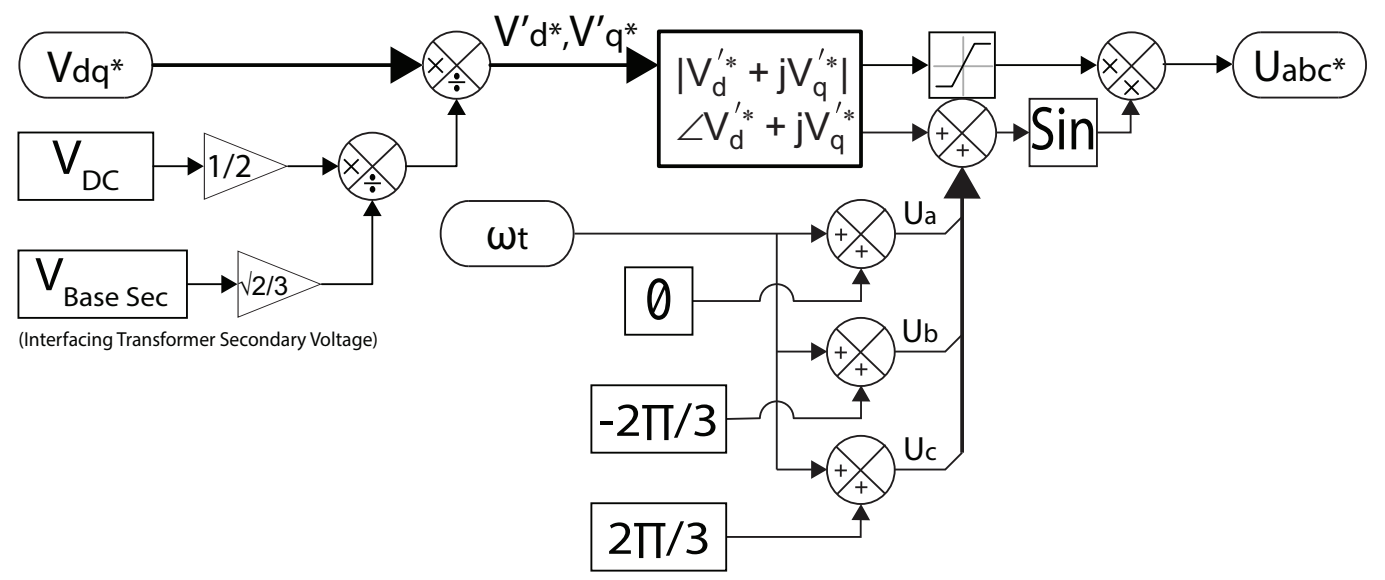

Figure 3.6: Grid-following IBR VSC reference signal generation 


\subsection{Fault response of IBR in grid-following mode}

IBR's fault response in grid following mode was tested by simulating a three-phase to ground fault at the inverter terminals when the microgrid was in gird connected mode. The inverter terminal voltage, output current, real, and reactive power output were monitored. Figure 3.7 shows that when the fault is initiated the voltage quickly drops to near 0 as expected for a bolted fault at the inverter terminal, the current controller's lag in the grid following mode can be seen as it takes the IBR 0.3s after the fault to reach its peak fault current value of $1.9 \mathrm{pu}$. It can also be seen that during the fault the IBRs real power output drops as the voltage drops to near 0 and the IBR starts supplying reactive power as required by IEEE 1547 standard. The reactive output is limited by due to the current limit combined with the reduced voltage.
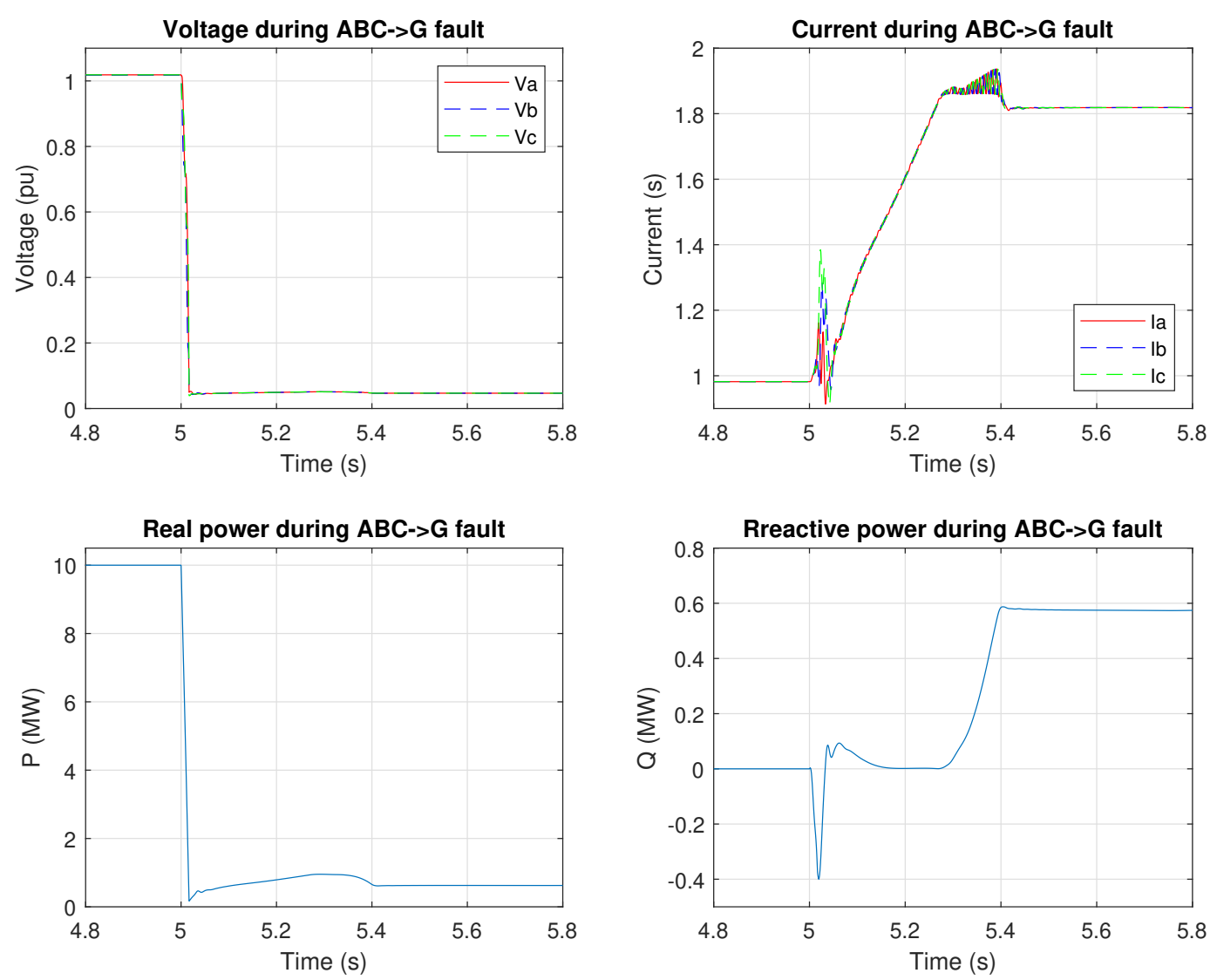

Figure 3.7: Fault response of grid following IBR 


\subsection{Impact of grid-following IBRs on the micro- grid in grid connected mode}

The Grid following inverter models were added to the LOHO system. The system was simulated with the IBRs to observe the impact of the distributed generation on the microgrid feeder. This case represents the grid-connected mode operation of the microgrid. In this case, the IBRs are supplying power at unity power factor, supplying the real power demand of the system and exporting the excess real power to the utility grid. The reactive power demand of the microgrid is supplied by the utility grid. The system line currents and power flow when IBRs are operating in grid following mode are shown in table 3.1 where, negative values for P and Q represent power flowing back to the grid. Table 3.2 shows the load currents when IBRs are operating in grid following mode.

Due to the large size of the IBRs in comparison to the system load there is a drastic change in the line flows in the system when comparing to the base case discussed in chapter 2. The power flow direction has changed for most lines, current magnitude also shows a drastic change. In this case with the IBRs connected the microgrid delivers $8335 \mathrm{~kW}$ of power to the utility grid while also supplying its own load. Before the IBRs were connected the system used to draws roughly $122 \mathrm{~A}$ of current on each phase with $1 \%$ unbalance at the low voltage side of the substation transformer. Now with the IBRs connected the microgrid delivers 390 A to the utility grid. In grid following mode the IBRs are operating at unity power factor, producing only real power.

In order to measure the fault currents supplied by the grid and IBRs in grid-connected microgrid mode faults were simulated on each bus of the system. Similarly to the base case, a 3phF, LLF on phase A-B, and SLGF on phase A were simulated on each bus 


\begin{tabular}{|l|l|l|l|l|l|}
\hline Line \# & IA (A) & IB (A) & IC (A) & P (kW) & Q (kVar) \\
\hline T1 $(\mathrm{HV})$ & 42.06 & 41.98 & 42.01 & -8335.00 & 791.80 \\
\hline 149 & 391.20 & 389.80 & 389.10 & -8396.00 & 367.40 \\
\hline 150 & 391.20 & 390.30 & 389.40 & -8402.00 & 366.60 \\
\hline 135 & 21.68 & 22.31 & 23.16 & 471.00 & 131.50 \\
\hline 105 & 7.79 & 7.95 & 6.44 & 172.30 & 219.70 \\
\hline $\mathrm{T} 2(\mathrm{HV})$ & 8.36 & 5.35 & 10.57 & -191.50 & 46.16 \\
\hline $\mathrm{T} 2(\mathrm{LV})$ & 217.90 & 217.80 & 275.60 & -192.80 & 39.66 \\
\hline 59 & 412.20 & 412.20 & 412.20 & -9002.00 & -319.50 \\
\hline 167 & 454.60 & 454.70 & 454.70 & -9992.00 & 13.45 \\
\hline 196 & 454.60 & 454.70 & 454.70 & -9995.00 & 3.94 \\
\hline 188 & 49.52 & 49.45 & 49.50 & 948.50 & -532.30 \\
\hline 97 & 49.47 & 49.40 & 49.45 & 948.60 & -530.80 \\
\hline 88 & 74.47 & 74.43 & 74.52 & 948.60 & 1335.00 \\
\hline 4 & 74.49 & 74.49 & 74.49 & 948.20 & 1335.00 \\
\hline 164 & 45.55 & 45.54 & 45.55 & -999.90 & -0.15 \\
\hline Inv3 10MW & 454.60 & 454.70 & 454.70 & -9999.00 & 0.00 \\
\hline Inv2 1 $\boldsymbol{W}$ & 45.55 & 45.54 & 45.55 & -999.90 & 0.00 \\
\hline Inv1 258kW & 309.10 & 309.00 & 308.50 & -258.10 & 0.00 \\
\hline
\end{tabular}

Table 3.1: Line currents and power flow for grid connected microgrid with gridfollowing mode IBRs

\begin{tabular}{|l|l|l|l|l|l|}
\hline Load \# & IA (A) & IB (A) & IC (A) & $\mathbf{P}(\mathbf{k W})$ & Q (kVar) \\
\hline 136 & 0.00 & 0.54 & 1.37 & 6.50 & 0.79 \\
\hline 139 & 0.00 & 0.33 & 0.06 & 2.77 & 0.57 \\
\hline 142 & 14.00 & 14.47 & 16.74 & 321.40 & 77.17 \\
\hline 133 & 15.13 & 15.29 & 16.77 & 340.60 & 36.70 \\
\hline 19 & 117.30 & 117.30 & 39.36 & 64.99 & 39.66 \\
\hline cap7 & 84.85 & 84.85 & 84.86 & 0.00 & -1865.00 \\
\hline 20 & 107.6 & 107.50 & 107.60 & 1948.00 & 1335.00 \\
\hline
\end{tabular}

Table 3.2: Load currents and power for grid connected microgrid with grid-following mode IBRs 
with a fault resistance of $0.01 \Omega$, a summary of the resulting fault currents is shown in table 3.3. The maximum fault current of $8196 \mathrm{~A}$ is observed after the substation as expected. The lowest fault current of $241 \mathrm{~A}$ was observed on bus 15 which is the farthest point on the feeder form the substation and is behind a transformer. While the peak fault current was reduced by roughly $100 \mathrm{~A}$ on average the fault currents in the microgrid with the grid following IBRs were higher due to the fault current contribution of the IBRs. Overall it can be seen that the grid still provides the bulk of the fault current in grid-connected mode with a small amount of fault current being supplied by the IBRs in grid following mode. Similar to the base case, the large amount of fault current compared to the load current was observed in almost all cases exceeding the load current by a factor of 20, however now the fault current is multi-sourced due to the presence of IBRs. In the event of a fault, those IBRs will also need to be isolated from the faults along with the utility grid.

\begin{tabular}{|l|l|l|l|}
\hline Bus \# & 3phF (A) & LLF (A) & SLGF (A) \\
\hline 2 & 8011.00 & 6968.00 & 8196.00 \\
\hline 11 & 8010.00 & 6966.00 & 8181.00 \\
\hline 5 & 3296.00 & 3042.00 & 2290.00 \\
\hline 9 & 3249.00 & 3010.00 & 2249.00 \\
\hline 10 & 1478.00 & 1332.00 & 1013.00 \\
\hline $15(\mathrm{LV})$ & 8320.00 & 6962.00 & 6273.00 \\
\hline $15(\mathrm{HV})$ & 320.26 & 267.98 & 241.46 \\
\hline 6 & 2770.00 & 2461.00 & 1875.00 \\
\hline 13 & 2869.00 & 2472.00 & 1645.00 \\
\hline 14 & 3240.00 & 2971.00 & 1942.00 \\
\hline 8 & 2641.00 & 2345.00 & 1728.00 \\
\hline 7 & 2631.00 & 2316.00 & 1709.00 \\
\hline 3 & 2612.00 & 2302.00 & 1683.00 \\
\hline 4 & 2596.00 & 2200.00 & 1637.00 \\
\hline 12 & 2590.00 & 2198.00 & 1635.00 \\
\hline
\end{tabular}

Table 3.3: Fault currents with grid-following mode IBRs 


\subsection{Grid-forming inverter model}

During islanded conditions, the inverters operate in grid-forming mode, where they provide voltage and frequency regulation for the islanded microgrid to operate properly. In this mode, the real and reactive power injections are based upon the conventional droop characteristics presented in [15]. The grid forming IBRs are modeled as average models using MATLAB's Simscape Electrical Specialized Power Systems library. Figure 3.8 shows the grid forming connection and overview diagram. The grid forming IBRs are connected to the microgrid through an LCL filter and an interfacing transformer. The grid forming IBR controller utilizes a CERTS control scheme described in [16]. A diagram of the controller is shown in figure 3.9. The controller

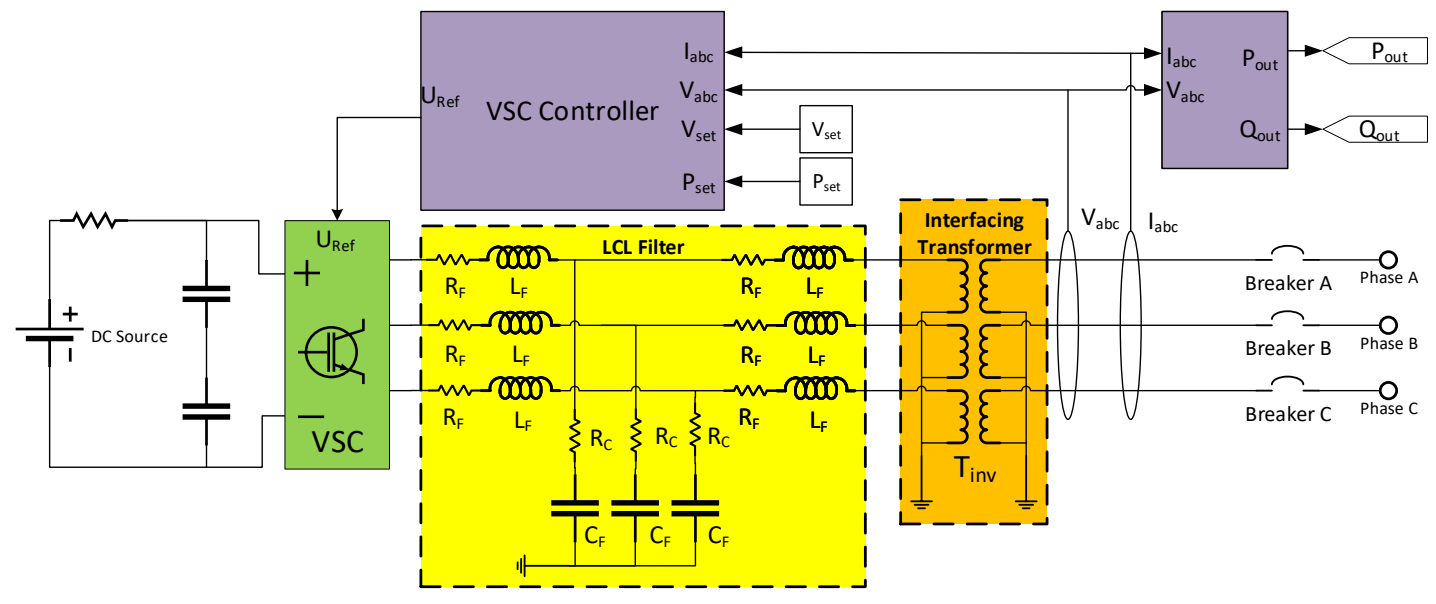

Figure 3.8: Grid-forming IBR connection diagram

transforms the measured output voltage $\left(V_{0}\right)$ and currents $\left(I_{0}\right)$ to the $\alpha \beta$ reference frame to obtain $V_{0 \alpha}, V_{0 \beta}, I_{0 \alpha}$ and $I_{0 \beta}$. The transformed parameters are used to calculate the power output using equation 3.2. The controller also calculates the peak voltage $\left(V_{\text {opeak }}\right)$ using the equation 3.3, which is used with a PI controller to regulate the voltage reference provided by the voltage droop stage, this improves voltage regulation by minimizing circulating currents throughout the system. The peak current $\left(I_{0 \text { peak }}\right)$ is calculated similarly to the peak voltage using the equation 3.4 , which will 
be used to control the fault current. A diagram of the calculations can be seen in figure 3.10 .

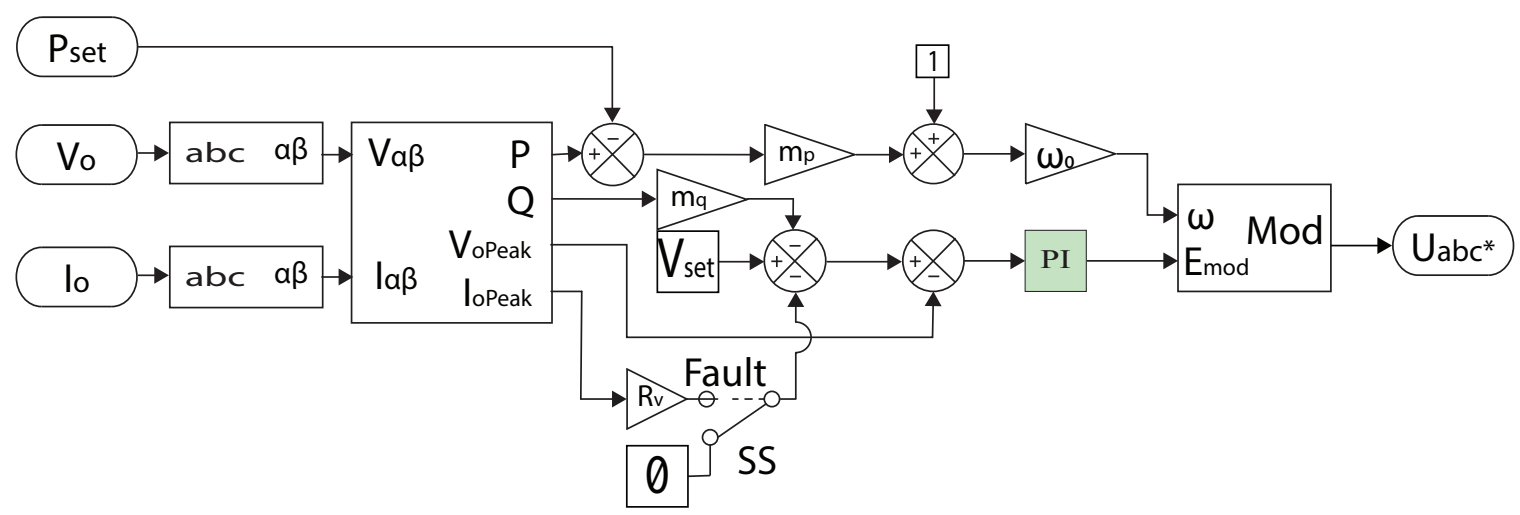

Figure 3.9: Grid-forming IBR controller

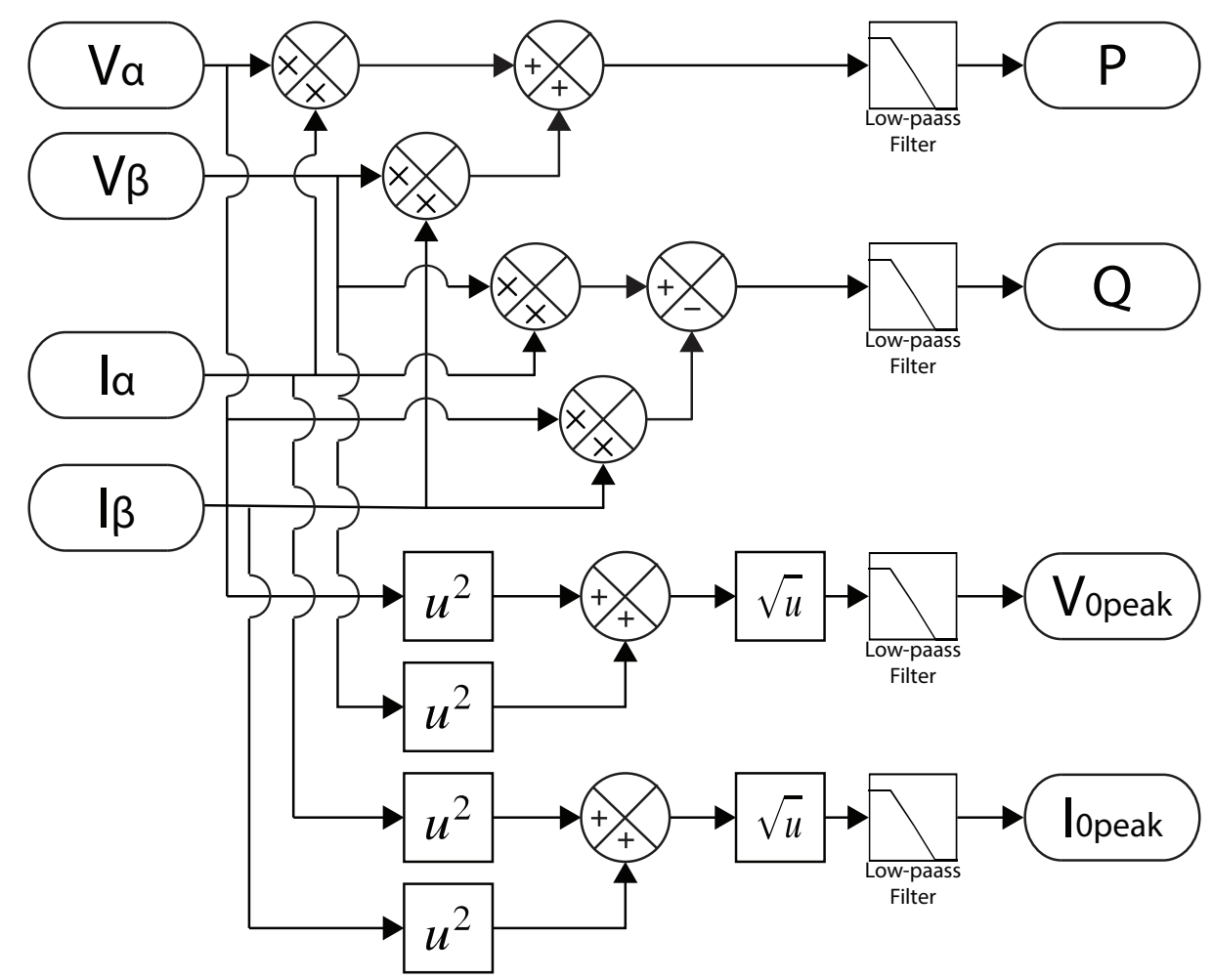

Figure 3.10: Grid-forming IBR power, voltage, current calculation

$$
\begin{array}{r}
P=\frac{3}{2}\left(V_{o \alpha} I_{o \alpha}+V_{o \beta} I_{o \beta}\right) \\
Q=\frac{3}{2}\left(-V_{o \alpha} I_{o \beta}+V_{o \beta} I_{o \alpha}\right)
\end{array}
$$




$$
\begin{gathered}
V_{0 \text { peak }}=\sqrt{V_{0 \alpha}^{2}+V_{0 \beta}^{2}} \\
I_{0 \text { peak }}=\sqrt{I_{0 \alpha}^{2}+I_{0 \beta}^{2}}
\end{gathered}
$$

After the real power, reactive power, and peak voltage are calculated a droop control is performed to using the equation 3.5 , where $m_{p}$ is the droop power-frequency characteristic. A voltage drop is also performed using the equation 3.6, where $m_{q}$ is the voltage-power droop characteristic. The output of the voltage droop stage is used by a PI-based voltage control to generate the amplitude for the reference signal $\left(E_{m o d}\right)$. The amplitude and $\omega$ from the droop control stage are used to create the modulation signals used to drive the IBR as shown in figure 3.11.

$$
\begin{gathered}
\omega=\omega_{\text {ref }}+m_{p} *\left(P_{\text {set }}-P\right) \\
V=V_{\text {set }}-\left(m_{q} * Q\right)
\end{gathered}
$$

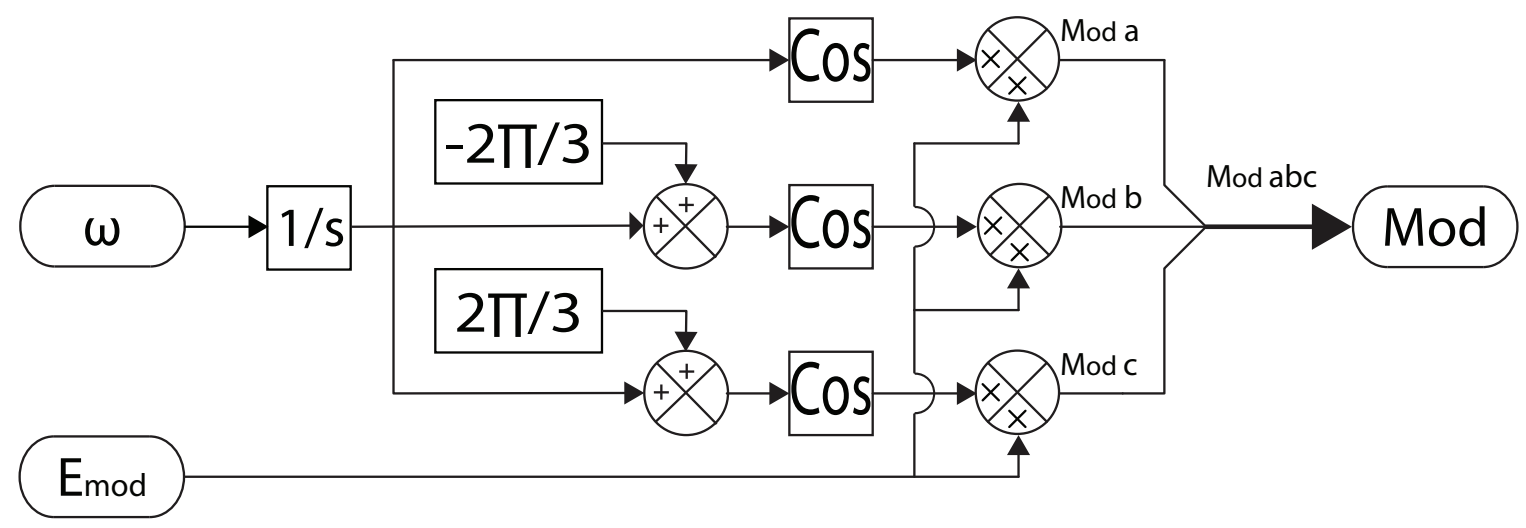

Figure 3.11: Grid-forming IBR modulation signal generation

A virtual impedance current limiting scheme discussed in [17] was used to limit the currents at $2 \mathrm{pu}$. The virtual impedance method is an alternative to the current reference limitation used in the grid following inverter. A transient virtual impedance such as a resistor, with two threshold values for the current amplitude, where the first threshold value activates the virtual impedance and the second disconnects the 
inverter in case of severe faults. Since the grid forming inverter should never disconnect from the microgrid the second threshold is ignored leaving only one threshold to activate the virtual impedance. When the current output of the inverter is below the threshold value, virtual impedance is not active. When the output current exceeds the threshold value the virtual resistance is activated so that the inverters output current is limited to below the maximum allowable current limit. This is achieved by reducing the commanded voltage reference generated by the droop control by modifying the droop control equation from equation 3.6 to equation 3.7, where $I_{t h}$ represents the threshold current and $R_{v}$ is the virtual impedance. For the the LOHO system the virtual impedance was chosen to be $R_{v}=0.7 p u$ and the threshold was set to $I_{t h}=1.2 p u$.

$$
V=V_{\text {set }}-\left(m_{q} * Q\right)-I_{0 \text { peak }} *\left\{\begin{array}{cc}
R_{v} & I_{0 \text { peak }} \geq I_{\text {th }} \\
0 & I_{0 \text { peak }} \leq I_{\text {th }}
\end{array}\right.
$$

\subsection{Grid-forming mode IBRs fault response}

In grid forming mode the IBRs need to act as the main source of fault current as the grid source is disconnected. However, fault current has to be limited in order to protect the inverter's electronics. The grid forming mode IBR's fault response was tested by simulating a three-phase to ground fault at the inverter terminals when the microgrid was in islanded mode. The inverter terminal voltage, output current, real, and reactive power output were monitored. Figure 3.12 shows that when the fault is initiated the voltage drops to near 0 as expected for a bolted fault at the inverter terminal. The current output increases dramatically at first as the filtering capacitor is drained and then is limited to below 2 pu by the virtual impedance. once the fault is cleared the system return back to steady-state. In comparison to the grid following inverter, the grid forming inverter model acts much more like a traditional generator, 
at the initiation of the fault the current rises quickly like a traditional generator unlike the grid following which gradually increased its current output.
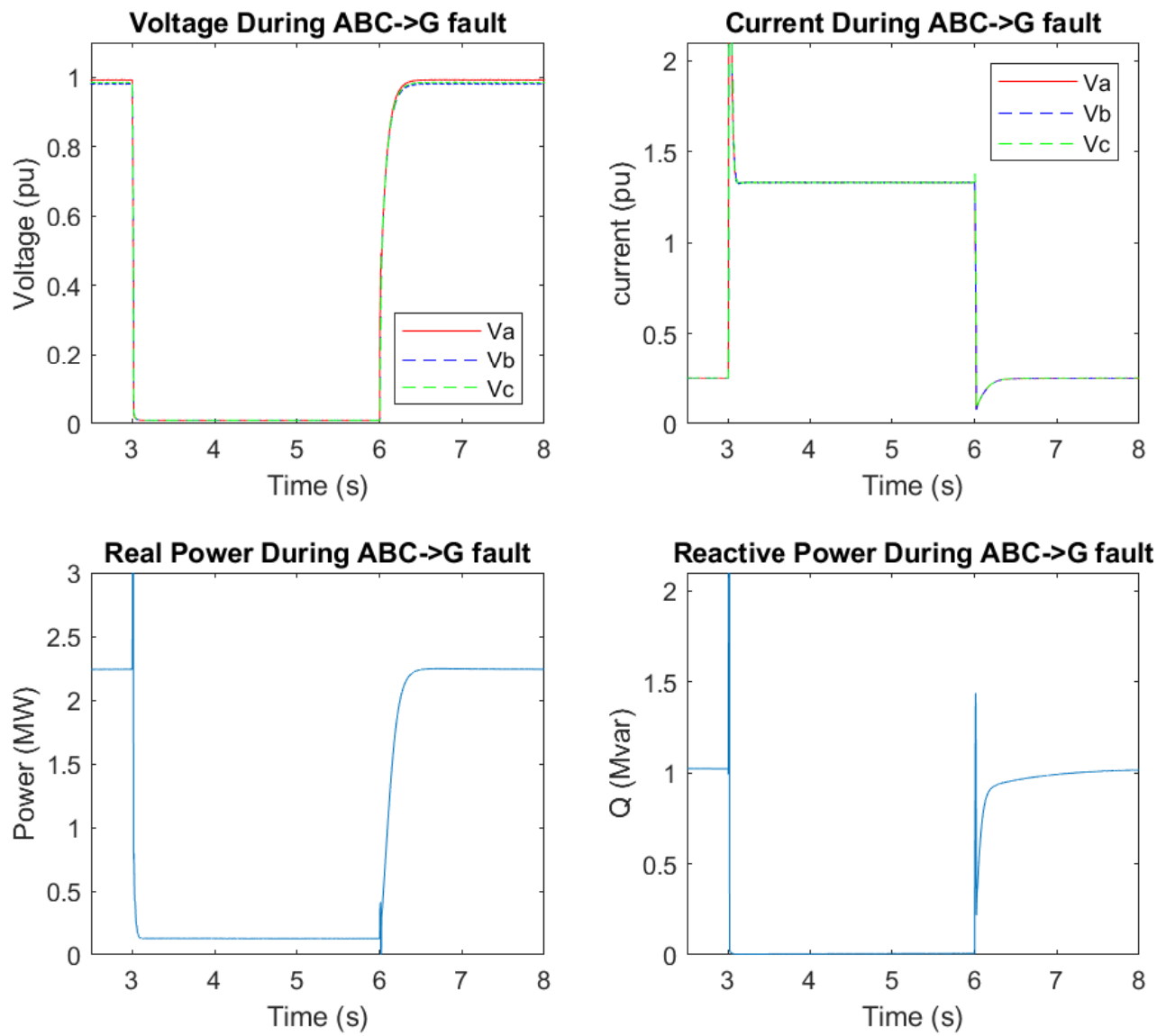

Figure 3.12: Grid-forming IBR fault response

\subsection{Impact of grid-forming IBRs on the microgrid in islanded mode}

The grid forming inverter models were added to the LOHO system. The system was simulated with the IBRs to observe the operation of the microgrid with only the distributed generation available. This case represents the islanded mode operation of the microgrid when the microgrid is disconnected from the grid source by opening the 
breaker located on bus 2. In this case, the IBRs are supplying both real and reactive power demand for the system. The IBRs use the droop control scheme to regulate their power output to meet the system demand and maintain healthy voltage and frequency. The system line currents and power flow when IBRs are operating in grid forming mode are shown in table 3.4 where negative values for P and Q represent power flowing towards the grid (not actually to the grid as its disconnected). Table 3.5 shows the load currents and load flow when IBRs are operating in grid forming mode.

\begin{tabular}{|l|l|l|l|l|l|}
\hline Line \# & IA (A) & IB (A) & IC (A) & P (kW) & Q (kVar) \\
\hline T1 (HV) & \multicolumn{2}{|l|}{ Disconnected } \\
\hline 149 & 4.63 & 3.06 & 4.04 & -57.97 & -57.84 \\
\hline 150 & 4.63 & 3.52 & 4.28 & -64.28 & -58.61 \\
\hline 135 & 28.76 & 29.42 & 30.63 & 628.5 & 19.96 \\
\hline 105 & 15.56 & 15.78 & 15.06 & 528.4 & 209.6 \\
\hline T2 $(\mathrm{HV})$ & 2.50 & 2.67 & 4.20 & 2.125 & -61.89 \\
\hline T2(LV) & 65.76 & 70.00 & 110.60 & 1.119 & -63.51 \\
\hline 59 & 32.51 & 32.71 & 33.31 & -695.4 & -56.4 \\
\hline 167 & 111.10 & 111.60 & 112.00 & -2280 & 655.9 \\
\hline 196 & 111.10 & 111.60 & 112.00 & -2280 & 655.5 \\
\hline 188 & 81.61 & 81.56 & 81.61 & 1584 & -707 \\
\hline 97 & 81.57 & 81.51 & 81.57 & 1583 & -707 \\
\hline 88 & 89.30 & 89.11 & 89.06 & 1584 & 1043 \\
\hline 4 & 89.31 & 89.13 & 89.08 & 1583 & 1042 \\
\hline 164 & 14.87 & 14.79 & 14.94 & -236.8 & -208.9 \\
\hline Inv3 10 MW & 111.10 & 111.60 & 112.00 & -2280 & 655.4 \\
\hline Inv2 1 MW & 14.87 & 14.79 & 14.94 & -236.8 & -208.8 \\
\hline Inv1 258 kW & 144.90 & 147.30 & 143.20 & -61.67 & -101.9 \\
\hline
\end{tabular}

Table 3.4: Line currents and power flow for islanded microgrid with grid-forming mode IBRs

Due to the lack of grid source the power flow direction for the microgrid is reversed in the islanded mode compared to the base case discussed in chapter 2. In the base case where all power and therefore currents were sourced for the grid, now the direction reverses as all power and currents are sourced from the IBRs located at the ends of 


\begin{tabular}{|l|l|l|l|l|l|}
\hline Load \# & IA (A) & IB (A) & IC (A) & P (kW) & Q (kVar) \\
\hline 136 & 0.00 & 0.53 & 0.37 & 6.31 & 0.77 \\
\hline 139 & 0.00 & 0.32 & 0.06 & 2.62 & 0.54 \\
\hline 142 & 13.61 & 14.06 & 16.28 & 303.70 & 67.97 \\
\hline 133 & 14.68 & 14.83 & 16.29 & 320.80 & 34.65 \\
\hline 19 & 115.40 & 115.20 & 38.69 & 62.79 & 38.44 \\
\hline cap7 & 82.30 & 82.36 & 82.17 & -0.36 & -1750.00 \\
\hline 20 & 104.1 & 103.90 & 104.00 & 1820.00 & 1251.00 \\
\hline
\end{tabular}

Table 3.5: Load currents and power for islanded microgrid with grid-forming mode IBRs

the feeder laterals. In the islanded mode case the inverters deliver $2578.47 \mathrm{~kW}$ of real power and absorb a total of $344.7 \mathrm{kVar}$ of reactive power output from the capacitor at bus 7 . In islanded mode, the IBRs easily supply the required amount of power to sustain the microgrid as the IBRs were sized in order to export power to the grid. In fact in islanded mode the IBRs limit their power output to maintain the system voltage and frequency. Table 3.6 shows the bus voltage during steady-state islanded microgrid operation. It can be seen that during steady-state operation the IBRs keep the voltage across the system within the specified IEEE 1547 DER standard [18]. IEEE 1547 standard states that the voltages must remain within $0.9 \mathrm{pu}$ and $1.1 \mathrm{pu}$ and the frequency mut be within $58.5 \mathrm{~Hz}$ and $60 \mathrm{~Hz}$.

In order to measure the fault currents supplied by the IBRs in islanded microgrid mode faults were simulated on each bus of the system. Similarly to the base case a 3phF, LLF on phase A-B and SLGF on phase A were simulated on each bus with a fault resistance of $0.01 \Omega$, a summary of the resulting fault currents is shown in table 3.7 .

The maximum fault current of $882 \mathrm{~A}$ is observed at bus 6 , rather than at the inverter terminals. The fault current is the highest at bus 6 rather than the largest inverters terminals due to the fault current limiting method of the inverters. The virtual 


\begin{tabular}{|c|c|c|c|}
\hline Bus \# & Va (pu) & $\mathrm{Vb}(\mathrm{pu})$ & Vc (pu) \\
\hline 1 & \multicolumn{3}{|c|}{ Disconnected } \\
\hline 2 & 0.997 & 0.997 & 0.998 \\
\hline 11 & 0.998 & 0.998 & 0.999 \\
\hline 5 & 0.998 & 0.998 & 0.999 \\
\hline 9 & 0.999 & 0.998 & 1.000 \\
\hline 10 & 0.998 & 0.999 & 0.999 \\
\hline $15(\mathrm{LV})$ & 1.000 & 1.000 & 1.008 \\
\hline 6 & 0.999 & 0.998 & 1.000 \\
\hline 13 & 0.999 & 0.998 & 1.000 \\
\hline 14 & 0.999 & 0.998 & 1.000 \\
\hline 8 & 1.000 & 0.999 & 1.000 \\
\hline 7 & 1.000 & 0.999 & 1.001 \\
\hline 3 & 1.000 & 0.999 & 1.001 \\
\hline 4 & 0.999 & 0.998 & 1.000 \\
\hline 12 & 0.998 & 0.997 & 1.000 \\
\hline
\end{tabular}

Table 3.6: Bus voltages for islanded microgrid with grid-forming mode IBRs

\begin{tabular}{|l|l|l|l|}
\hline Bus \# & 3phF (A) & LLF (A) & SLGF (A) \\
\hline 2 & 638 & 815 & 334 \\
\hline 11 & 638 & 815 & 334 \\
\hline 5 & 667 & 867 & 365 \\
\hline 9 & 664 & 866 & 364 \\
\hline 10 & 595 & 758 & 308 \\
\hline $15(\mathrm{LV})$ & 3275 & 3305 & 1177 \\
\hline 15 & 126 & 127 & 65 \\
\hline 6 & 693 & 882 & 374 \\
\hline 13 & 695 & 882 & 374 \\
\hline 14 & 695 & 882 & 374 \\
\hline 8 & 686 & 878 & 374 \\
\hline 7 & 685 & 877 & 374 \\
\hline 3 & 683 & 875 & 374 \\
\hline 4 & 677 & 872 & 374 \\
\hline 12 & 677 & 871 & 374 \\
\hline
\end{tabular}

Table 3.7: Fault currents with grid forming mode IBRs in islanded microgrid state 
impedance fault current limits the fault current to a lower value when the voltage drop is more severe, therefore for fault at the inverter terminals the fault current contribution from that inverter is limited to a lesser value compared to a fault a few lines away from the inverter terminals. The lowest fault current of $65 \mathrm{~A}$ was observed on bus 15 due to low fault current contributions from the largest inverters as they are furthest from bus 15 and the inverter at bus 15 is limited to a maximum of roughly $23 \mathrm{~A}$ of fault current contribution. Compared to the base case and the gird connected case the fault currents in the islanded mode are considerably lower. Due to the lack of a strong grid source, on average the fault currents in the islanded microgrid with the grid forming IBRs are typically an order of magnitude lower. Overall it can be seen that unlike the base case where a large amount of fault current compared to the load current was present, in islanded mode many of the fault current values are closer to load current. For example for a 3ph fault on bus 15 the fault current observed by the recloser REC in grid connected mode is $1013 \mathrm{~A}$, in islanded mode its reduced to $120 \mathrm{~A}$. However, due to the large size of the IBRs compared to the load on the system, there is still sufficient fault current to use OC relays. 


\section{Chapter 4}

\section{Analysis of Existing Protection Devices}

Traditional protection devices such as overcurrent relays, Differential Relays, and Distance relays each face unique challenges when present in a microgrid setting [19].

\subsubsection{Overcurrent Elements}

Overcurrent relays and other overcurrent based protection devices suffer from large coordination ranges due to the switching nature of a microgrid, where a microgrid can switch from grid-connected to islanded state and vice-versa either intentionally or unintentionally. The switch from one state to another changes the sources of fault current available to the system and thus the amount of fault current observed by an OC relay element. Generally, the fault current available in grid-connected mode is many times greater than the fault current available in islanded mode, leading to large coordination ranges for OC based protection devices [19]. 


\subsubsection{Differential Protection}

Differential protection offers a solution that is free from the issues faced by conventional OC based protection; however, differential protection has its own shortcomings. Differential protection of feeders requires data sharing between devices either through digital communication or through physical wires between devices to achieve coordination. This may be feasible for geographically small microgrids [19]. Differential protection for a microgrid, while feasible in principle, in actuality can become impractical due to the cost associated with the intensive communication needed for differential protection. In general, cost of differential protection is higher compared to other protective devices.

\subsubsection{Distance Protection}

Distance protection also offers a solution that is free from fault current fluctuations; however, it does not lend itself naturally to radial distribution feeders with tapped load laterals. Distribution feeders such as the LOHO system shown in figure 2.1, can cause the distance relays to under-reach due to current infeeds and can also suffer

from blind spots due to the presence of DER. Additionally, depending on the DER and fault location, a distance relay may not even see the fault. It is conventional for a tapped feeder to either be protected by a differential scheme, or a distance scheme using intertripping. A communication network between the devices is necessary for intertripping; therefore, there is little to no advantage to using distance protection over differential protection in this scenario [19]. 


\subsection{Issues faced by the existing protection in the LOHO system}

As per the standard protection methods for distribution feeders, the test feeder is protected using a relay at the substation, a recloser, a sectionalizer, and load fuses, as shown in Fig. 2.1. Overcurrent (OC) relay $R 1$ with reclosing function is located at bus 2 and is coordinated with the sectionalizer $S E C$ at bus 8 and the recloser $R E C$ at bus 5 . The loads are protected using appropriate load fuses. The fuses and relays are set to operate for permanent faults. The recloser and sectionalizer are set to save fuses by clearing temporary faults. While these devices adequately protect the feeder when it is only fed by the grid, they cannot protect the proposed microgrid due to reasons outlined in this section.

\subsubsection{Bidirectional current}

Multiple sources create bidirectional fault currents, making simple overcurrent based protection difficult (or impossible) in many situations. Figure 4.1 highlights the issues caused by multiple sources and bidirectional fault currents; fault currents for a fault on bus 9 are shown in red and fault currents for a fault on Line 149 are shown in green. Taking the recloser "REC" as an example to illustrate the issue of bidirectional fault current. The recloser $R E C$ is only supposed to operate for faults downstream on buses $9,10,15$, and load laterals associated with these buses as per conventional protection. These faults would all have a similar fault current path as the fault on bus 9 from the perspective of the recloser. However, when IBR is added at bus 15, that recloser now also needs to operate for upstream faults on bus 2, bus 11, line 149 line and 150 in order to isolate the IBR on bus 15 from the fault-point. These faults would 


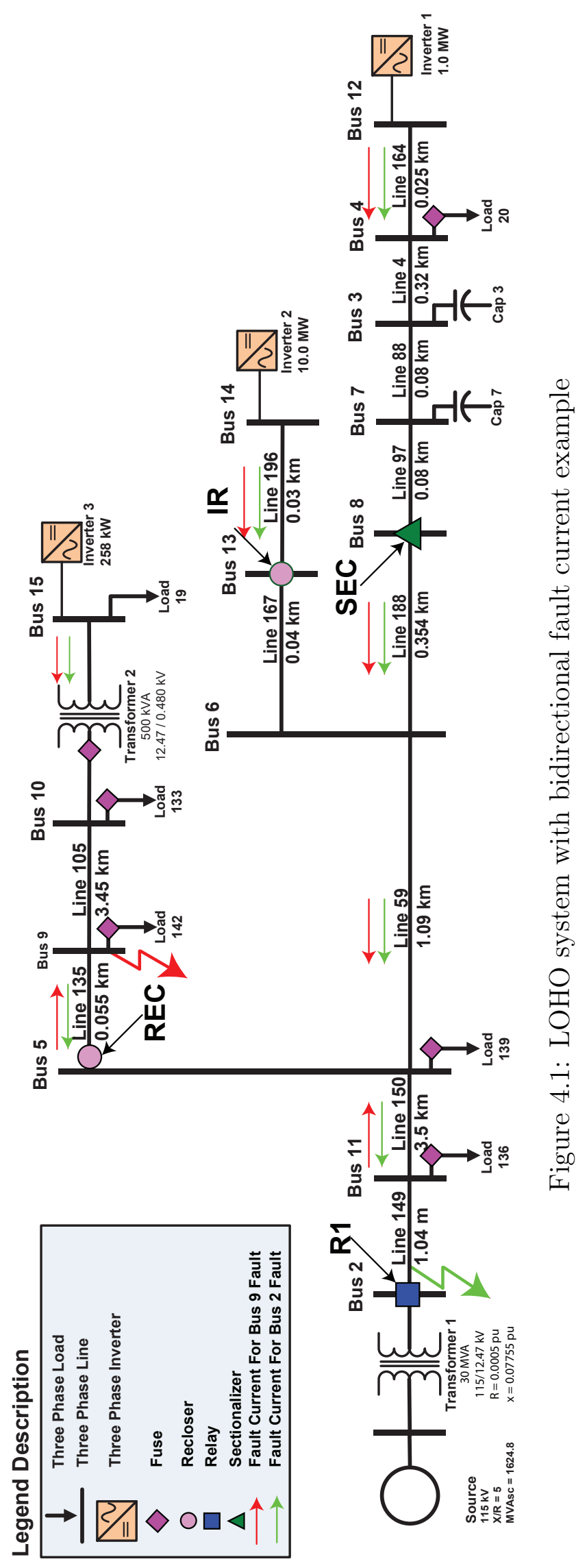


in general follow the fault path shown in green for a fault on line 149. The recloser now must operate for both types of faults upstream and downstream with reverse and forward fault current respectively. Thus, as the system changes from single-sourced to multi-sourced, the protection also needs to go from simple OC to at least directional OC. This often requires different settings for both directions because fault current from IBR for upstream faults may be very limited. Taking the same recloser as an example, the recloser sees a load current of approximately 30 A flowing towards bus 9 from bus 5 . However, depending on the fault location and fault resistance the current observed by the recloser can drop to as low as $10 \mathrm{~A}$ in the reverse direction as the $258 \mathrm{~kW}$ inverter feeds the faults upstream from the recloser. Similarly, the Relay R1 at bus 2 needs to be able to isolate the microgrid from the utility system if a fault occurs on the utility grid but continue to operate for downstream faults at bus 5, 6 , 8, 11, and 13. Applying the same logic to the rest of the system with three IBRs, the existing protection fails to isolate the faults on buses $3,4,5,6,7$ and 8. Protection must be enhanced to isolate faults from the IBR-side.

Bidirectional currents can also make existing devices misoperate. For example, sectionalizer $S E C$ at bus 8 (see Fig. 4.1), which previously operated in conjugation with the relay $R 1$, cannot be coordinated with two more reclosers $R E C$ and $I R$ for faults downstream of $S E C$. The sectionalizer also cannot be coordinated with any device downstream in order to operate for faults upstream such as the fault on Line 149 shown in green in figure 4.1. Additionally, the sectionalizer could miscount due to the low fault current contribution from the DER on bus 12 being interpreted as load current in the event of an upstream fault. Therefore, the sectionilizer is removed and replaced with a directional overcurrent relay $R 3$ to provide better selectivity to the proposed protection. Additionally relays $R 2$ and $R 4$ shown in Fig. 4.3 are added to the existing protection scheme to ensure faults could be isolated from the IBR-side. A relay could be added at bus 14 as well; however, because the utility had already 
added an intelligent recloser at bus 13 and no load is present on bus 14, it was kept at bus 13 as it severs essentially the same role.

\subsubsection{Low fault current}

Since IBRs limit their current output to low values ( $2 \mathrm{pu}$ in this study), they pose challenges for device coordination in both grid-connected and islanded modes. This is true especially in islanded mode due to lack of adequate fault currents. For example, the maximum fault current in the grid-connected mode for the feeder is around 8000 A. However, the majority of the fault current in grid-connected mode is from the grid. The fault current contribution from the inverters is limited to a maximum of $93 \mathrm{~A}, 925 \mathrm{~A}$, and $24 \mathrm{~A}$ for the $1 \mathrm{MW}, 10 \mathrm{MW}$, and $258 \mathrm{~kW}$ inverters, respectively. Low fault current from the inverters creates challenges in fault detection from the inverter side as the fault current from the inverters can be similar to or even lower than load current. For example, for a fault on bus 11 shown in figure 4.2, in gridconnected mode the total fault current is 5489 A. However, majority of that fault current is sourced from the grid while the DERs only supply a combined $620 \mathrm{~A}$ of fault current. Additionally, $591 \mathrm{~A}$ of the DER supplied fault current is sourced from inverter 2, with only $12 \mathrm{~A}$ and $21 \mathrm{~A}$ of fault current being sourced from inverter 1 and inverter 3 respectively. The fault current observed at bus 8 is lower than the nominal load current at bus 8 , This makes fault detection using conventional non-direction overcurrent relays impossible. Therefore, in the presence of DER, at least directional overcurrent relays must be used.

The maximum fault current in the islanded mode is reduced to roughly $880 \mathrm{~A}$. The low fault current during islanded mode can lead to difficulties in fault detection or

long tripping times for OC elements if settings are based on fault currents observed for grid-connected mode. On the other hand, if settings are based on fault currents 


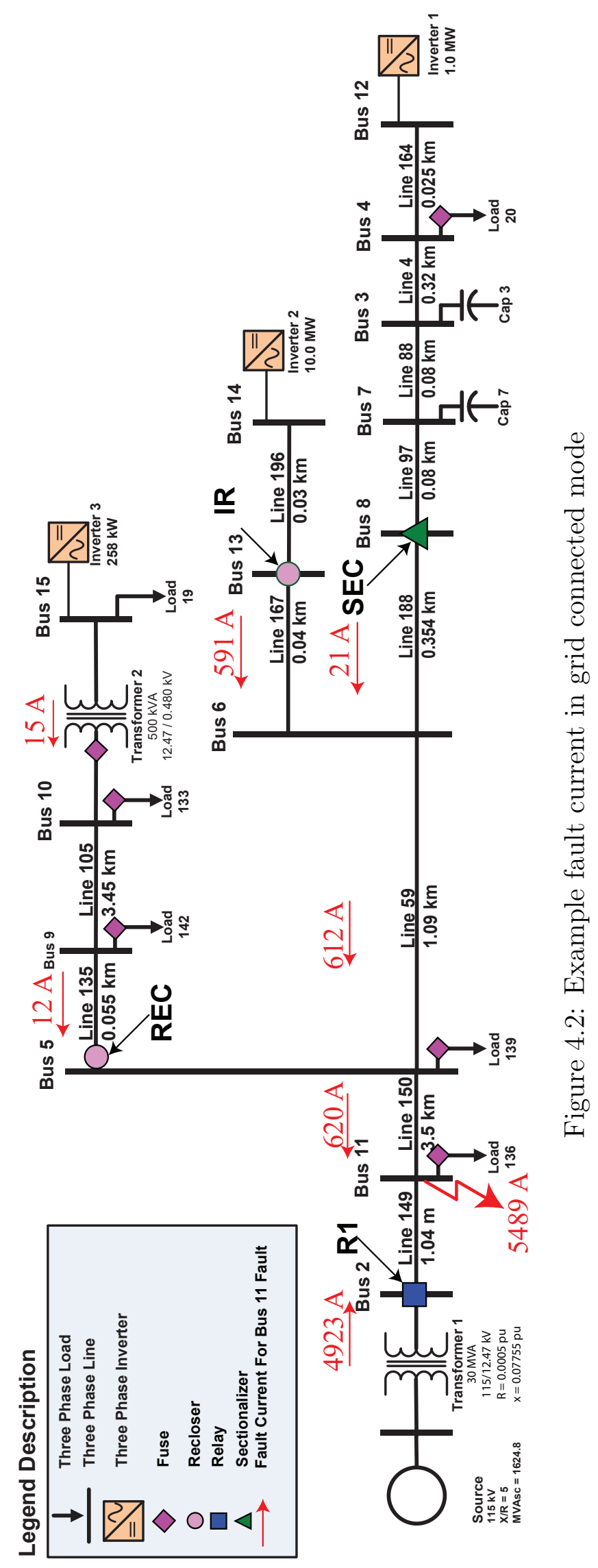


observed in islanded mode, the $\mathrm{OC}$ element can misoperate or fail to coordinate properly in grid-connected mode.

\subsubsection{Large coordination ranges}

Due to the limited fault currents from IBRs, coordination ranges can become large and lead to difficulties in coordination, often resulting in excessive operating time, as illustrated in [19]. In the test case under discussion, the coordination range for $R E C$ can span from 3500 A to 120 A due to large differences in fault current profiles in grid-connected and islanded modes.

\subsection{Summary of updates to the protection devices}

Based on the issues discussed in section 4.1 several changes were made to the LOHO systems protection devices. First, and most importantly, all devices were made to be directional so settings can be set for both upstream (reverse) and downstream (forward) direction faults. Directional overcurrent relays were placed at the IBR terminals for inverter 1 and inverter 3 , inverter 2 did not have a relay placed at its terminals as it already has an intelligent recloser on bus 13. The sectionalizer was replaced with a directional OC relay. The changes to the protection devices essentially split the system into 5 protection zones highlighted in figure 4.3. 


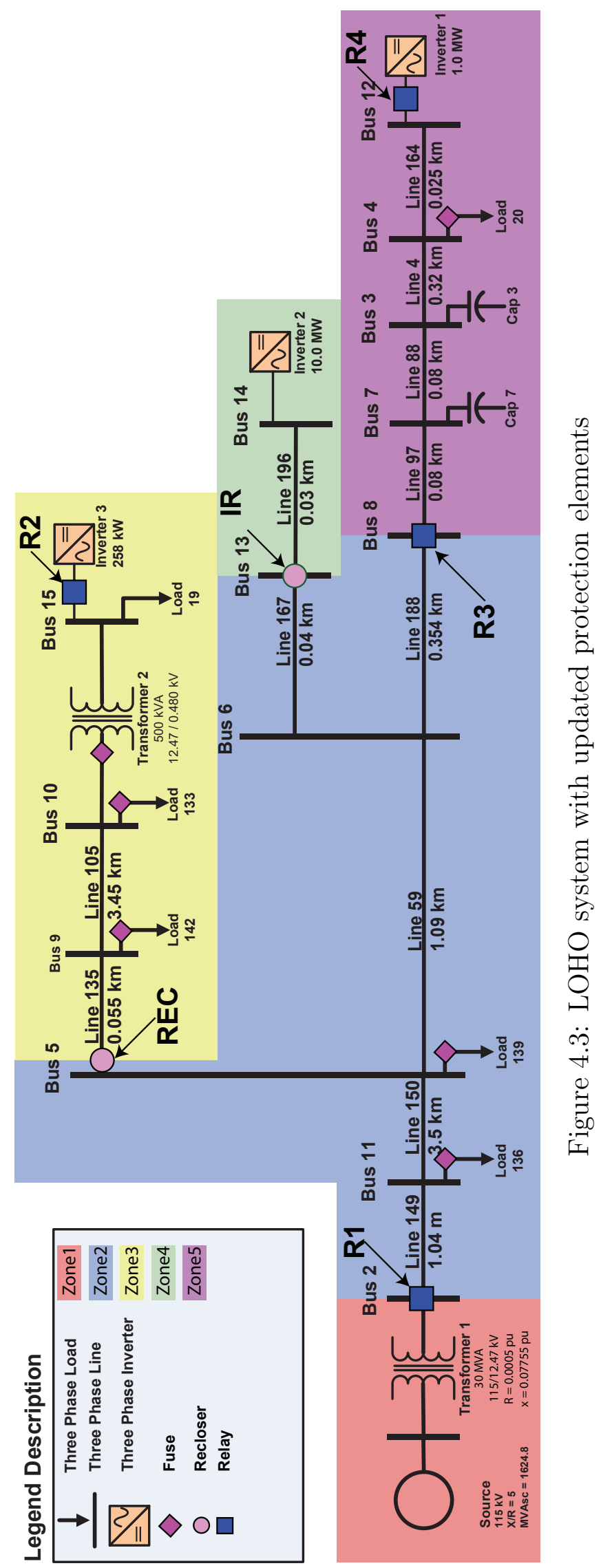




\section{Chapter 5}

\section{Proposed Protection Method}

It is clear from the discussion in Section 4.1.1 that bidirectional protective devices are needed to protect the microgrid. It is also clear that settings will need to be different for different operating modes due to a large difference in fault currents, thus requiring an adaptive design. Based on the available protective devices, the microgrid was divided into 5 protection zones as shown in Fig. 4.3. Relays $R 1, R 2$, and reclosers $R E C$ and $I R$ will have different settings for different fault-current directions. Also, all devices will have different settings for grid-connected mode and islanded mode.

For the purpose of this discussion, the direction downstream of the feeder will be called "forward" and the direction upstream will be called "reverse". Numerical relay manufacturers have relays and other protective devices that can store multiple settings that can be activated and deactivated using communication. For example, the SEL 751 relays have the capacity to set the max phase time overcurrent with directional control $(51 \mathrm{P})$. These relays also allow the user to create the logic which is used to generate a trip signal based on the output of different relaying elements such as inverse-time overcurrent, under-voltage, and directional. Therefore, a relay can be set to operate, for example, if the directional element indicates a forward current and 
the OC criteria are met, and the voltage has dropped below the set threshold. Using these programmable trip conditions and multiple group settings, the same relay can be adaptively programmed to operate differently for different modes of operation. A minimum of 2 setting groups should be stored in a relay, 1 for grid-connected mode, and 1 for islanded mode. Each setting group is further broken down into settings for forward (F) and reverse (R) direction. This results in 4 sets of Minimum Operating Current (MOC) settings and Time Dial (TDs) settings for each device that needs to operate for fault current in both directions as shown in figure 5.1 [20]. A flow chart of which settings are active and how a trip signal is generated is shown in figure 5.2.

Depending on the microgrid operating mode, either the grid-connected or islanded mode settings will be active. The signal used to switch the inverter operating mode can be used to activate/deactivate the appropriate mode-setting. In the case of loss of signal, relays can be set up either to trip or to default to islanded mode protection. If the relay is tripped due to loss of signal then the breaker associated with the relay is opened, isolating the section of the system protected by that relay. If the relays default to islanded mode protection then the relays could trip unintentionally trip during overload conditions however, they will still be able to isolate any fault that occurs in the system. This is preferable to being an able to isolate a fault if a fault occurs.

In islanded mode, faults are isolated by the operation of the appropriate devices using their islanded mode setting by isolating the faulted section of the microgrid. If the faulted section includes a DER, then the DER will be isolated by the protective elements placed on the DER connection terminals (Relays $R 2, R 4$, and $I R$ ). In gridconnected mode, there are two options for isolating faults inside the microgrid. Relay $R 1$ can be set to operate instantaneously to disconnect the grid, transitioning the microgrid into islanded mode and isolating the faulted zone based on settings for the 


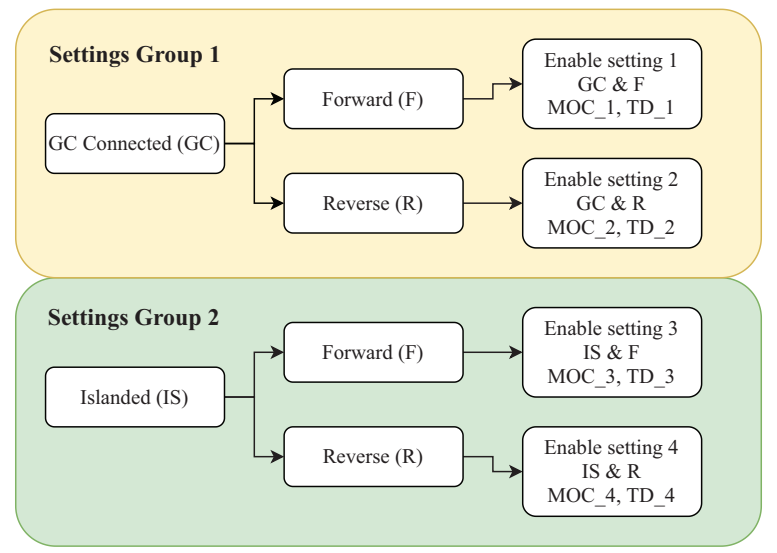

Figure 5.1: Settings groups for bidirectional protective devices.

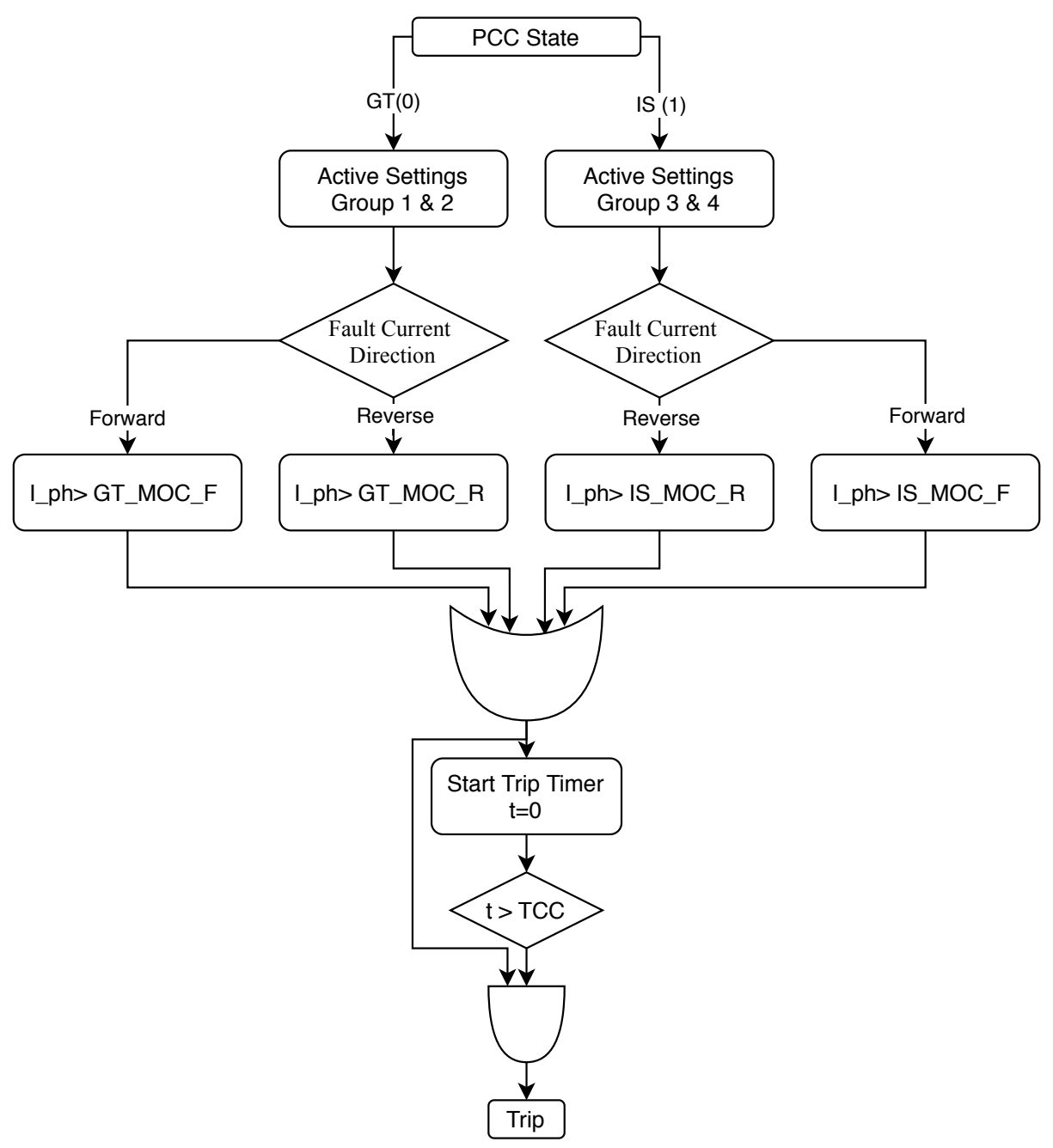

Figure 5.2: Flow chart of relay trip signal generation 
islanded mode. Alternatively, $R 1$ can coordinate with the devices inside the microgrid to isolate the faulted zone. The first option is more likely, as utilities do not coordinate with the protection of customer-owned systems. However, as microgrids proliferate, it is likely that microgrids may be owned by utilities, in which case, the second option may become feasible. Both options will be discussed here. For the second option, for the microgrid under study, $R 1$ will backup $R 3, I R$, and $R E C$ for faults in zones 5 , 4 , and 3 , respectively.

\subsection{Example settings calculation}

As an example for a fault on bus 9 in islanded mode, the recloser RECs forward direction $\left(R E C_{F}\right)$ setting needs to operate. In islanded mode, REC has a load current of $35 \mathrm{~A}$ with a fault current ranging from $120 \mathrm{~A}$ to $950 \mathrm{~A}$. Therefore the minimum operating current (MOC) for $R E C_{F}$ is set to 1.5 times load current at $53 \mathrm{~A}$. As $R E C_{F}$ is set to allow for fuse saving with the fuse protecting transformer T2, operating time of $0.30 \mathrm{~s}$ and $0.03 \mathrm{~s}$ are chosen for the slow and fast curves respectively, based on the operating time of the fuse for the coordination range of $120 \mathrm{~A}-610 \mathrm{~A}$. Based on the chosen operating time $(\mathrm{Ot}), \mathrm{MOC}$, and coordination range, a curve is selected from the set of U.S TCC curves. Equations 5.1 - 5.5 representing U1: Moderately Inverse (MI), U2: Inverse (I), U3: Very Inverse (VI), U4: Extremely Inverse (EI), and U5: Short-Time Inverse (STI) respectively are used to calculate the time dial setting for the protection devices, where $t_{p}$ is the operating time, $T D$ is the time-dial setting and $M$ is the multiples of pickup current. For $R E C_{F}$ fast curve a Short-Time Inverse (STI) Curve is selected with a Time Dial setting (TDS) of 0.5 is chosen and for the slow curve, an Extremely Inverse (VI) curve is selected with a TDS of 2.250. 


$$
\begin{gathered}
t_{p}=T D *\left(0.02260+\frac{0.0104}{M^{0.02}-1}\right) \\
t_{p}=T D *\left(0.18000+\frac{5.95}{M^{2}-1}\right) \\
t_{p}=T D *\left(0.09630+\frac{3.88}{M^{2}-1}\right) \\
t_{p}=T D *\left(0.03520+\frac{5.67}{M^{2}-1}\right) \\
t_{p}=T D *\left(0.00262+\frac{0.00342}{M^{0.02}-1}\right)
\end{gathered}
$$

Similarly, for a fault on bus 5, the recloser RECs reverse direction $R E C_{R}$ needs to operate; however, this time the fault current seen by the recloser is limited to $15 \mathrm{~A}$ 20 A. This is due to the low fault current capacity of the Inverter1. Also, in this case $R E C_{R}$ needs to backup $R 3_{F}$ and $I R_{F}$. As the load current $R E C_{R}$ is 0 (load current in islanded mode should always flow in the forward direction for $R E C$ ), MOC for $R E C_{F}$ is set to $5 \mathrm{~A}$, and the Ot is set to be the $0.3 \mathrm{~s}$ more than the Ot for $R 3_{F}$ or $I R_{F}$, whichever is greater. Operating time of $0.7 \mathrm{~s}$ and $0.1 \mathrm{~s}$ are chosen for the slow and fast curves respectively. Based on the operating curves and the different coordination ranges for both $R 3_{F}$ and $I R_{F}$ along with the MOC, for the fast curve, an STI curve with a TDS of 1.02 is chosen and for the slow curve, an STI curve with a time dial setting of 7.25 is chosen.

In grid-connected mode, RECs forward direction $\left(R E C_{F}\right)$ setting needs to operate for faults on bus 9 , bus 10, line 135, and line 105. REC has a load current of 35 $\mathrm{A}$ in the forward direction when the IBRs are disconnected or not producing power and $25 \mathrm{~A}$ when IBRs are connected and operational. Also, the fault current in the forward direction ranges from 1013 A - 3290 A. Therefore, the minimum operating current (MOC) for $R E C_{F}$ is set to 1.5 times the load current for the higher of the 
2 grid-connected cases at $60 \mathrm{~A}$. As $R E C_{F}$ is set to allow for fuse saving with the fuse protecting transformer T2. Operating time of $0.5 \mathrm{~s}$ and $0.03 \mathrm{~s}$ are chosen for the slow and fast curves respectively, based on the operating time of the fuse for the coordination range of $235 \mathrm{~A}$ - $350 \mathrm{~A}$. Based on the chosen operating time (Ot), MOC, and coordination range, a curve is selected and the TDS for the fast and slow curves is calculated for the recloser in grid-connected mode. For $R E C_{F}$ fast curve, a ShortTime Inverse (STI) Curve is selected with a Time Dial setting (TDS) of 0.5 and for the slow curve, a Short-Time Inverse (STI) curve is selected with a calculated TDS of 5.53 .

In grid-connected mode, RECs reverse direction $\left(R E C_{R}\right)$ setting needs to operate for faults on bus 2 , bus 11 , line 149 , and line 150 and backup the $R 3_{F}$ and $I R_{F}$. Also, the fault current observed in reverse direction ranges from $10 \mathrm{~A}$ to $30 \mathrm{~A}$ supplied by the Inverter1 located at bus 15 . The load current $R E C_{R}$ is 0 (load current in the grid-connected mode in steady-state should always flow in the forward direction); therefore, $\mathrm{MOC}$ for $R E C_{F}$ is set to $5 \mathrm{~A}$, and the $\mathrm{Ot}$ is set to be the $0.3 \mathrm{~s}$ more than the OT for $R 3_{F}$ or $I R_{F}$, whichever is greater. Operating time of $0.7 \mathrm{~s}$ and $0.1 \mathrm{~s}$ are chosen for the slow and fast curves respectively. Based on the operating curves and the different coordination ranges for both $R 3_{F}$ and $I R_{F}$ along with the MOC; for the fast curve, an STI curve with a TDS of 1.57 is chosen and for the slow curve, an STI curve with a TDS of 7.27 is chosen. A summary of the recloser REC settings is shown in table 5.1.

Additionally, for each setting group calculated in this section, an Instantaneous trip (IT) setting is calculated when possible. If the maximum fault current seen by the device is at least 1.3 times greater than the maximum coordination range, then the instantaneous trip was set to the larger of 6 times the load current or 1.25 times the maximum coordination range. For the case of the recloser $R E C$ in grid-connected 
mode in forward direction, the maximum fault current of $3290 \mathrm{~A}$ is much larger than the max coordination range of 390 A coordinating with the Transformer Fuse F2, therefore the instantaneous trip is set to $1.25 * 390 \approx 487$.

For reverse direction setting in grid-connected mode, the maximum fault current is observed to be 30 A from the DER on bus 15 with no load current in the reverse direction. In grid-connected mode, load current always flows in the forward direction across the recloser. Traditionally no instantaneous setting would be set because the max coordination range is the same as the max fault current. Since any current in the reverse direction is likely due to a fault, an IT setting is set for this special case at $1.25 * 390 \approx 38 \mathrm{~A}$. In islanded mode, the maximum fault current observed in the forward direction is $950 \mathrm{~A}$ with a load current of $35 \mathrm{~A}$ and a coordination range of 125$610 \mathrm{~A}$. The ratio of the maximum fault current to the maximum coordination range is greater than 1.3 therefore, the instantaneous trip was set at $1.25 * 610 \approx 762 \mathrm{~A}$.

Similarly to the reverse direction case in grid-connected mode, in islanded mode IT for the reverse direction was set at $38 \mathrm{~A}$. Figure 5.3, 5.4, 5.5, and 5.6 show the coordinated TCC curves for recloser REC in grid-connected forward, grid-connected reverse, islanded forward, and islanded reverse setting respectively. In all the TCC curves figures, the coordination ranges and other protection devices curves are shifted to account for the different fault currents seen by the individual devices. For example, when the recloser $R E C_{R}$ is backing up $R 3_{F}$ for a fault on bus 7 , the fault current seen by the recloser $R 3_{F}$ is a summation of the currents seen by $R E C_{R}$ and $I R_{R}$. Therefore, the x-axis of the TCC curves will be miss-aligned and need to be shifted. Figures 5.3 - 5.6 are shifted to align with TCC curves of the recloser $R E C$ using the ratio of the main device ( $R E C$ in this figures) fault current to the secondary device fault current (IR, $R 3$ and $F 2$ in this figures).

Using the setting in table 5.1 for a $3 \mathrm{ph}$ fault on bus 10 in grid-connected mode, the 
fault current seen by the recloser is in the forward direction and has a magnitude of $1450 \mathrm{~A}$. In this situation, settings corresponding to mode $G C$ and direction $F$ in the table will be activated, which translates to MOC of $60 \mathrm{~A}$, and TDS of 5.53 and 0.5 for the fast and slow curves, respectively. The inverse time OC element will activate when the current exceeds the MOC and will trip after a time delay dictated by the TDS setting. However, in this case, the fault current is sufficiently large to activate the instantaneous trip, operating as quickly as possible.

Similarly, to the method shown in this section, the 4 sets of settings for each device can be calculated based on the fault currents observed by each device. The coordination between devices and their settings will be covered in section 5.2 and 5.3 .

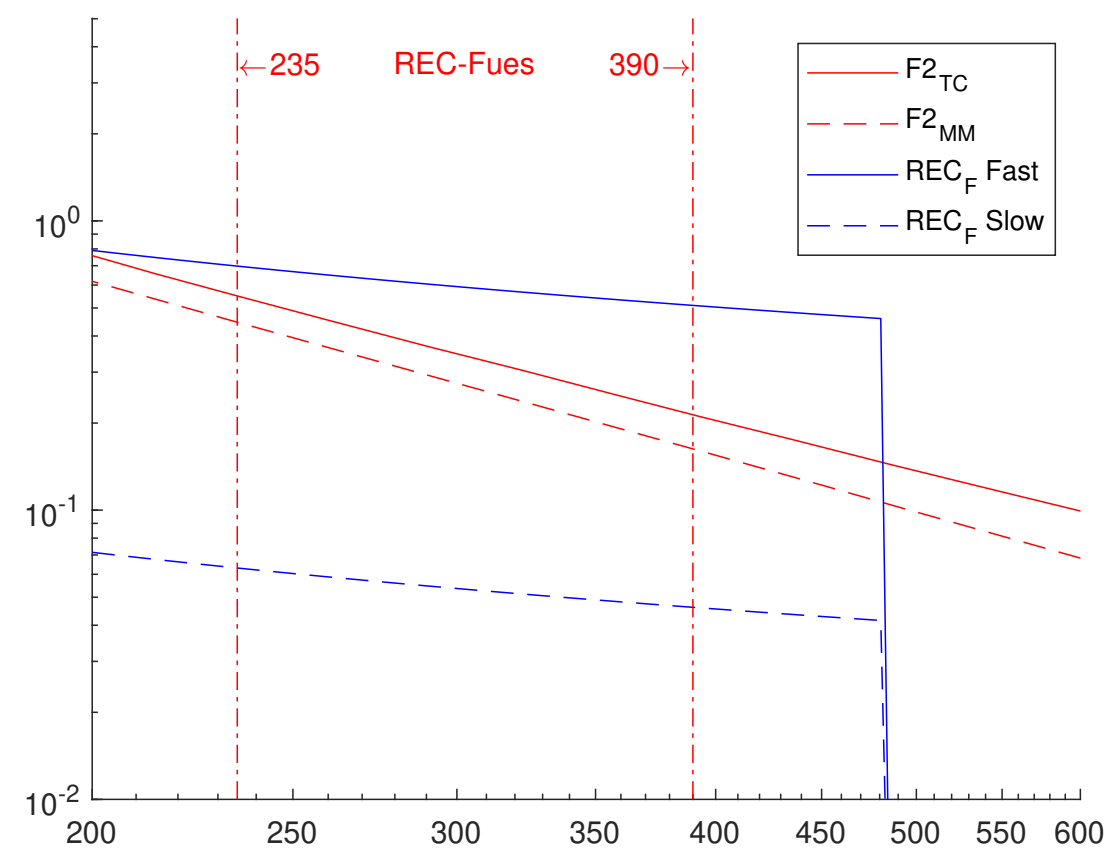

Figure 5.3: Grid connected $R E C_{F}$ TCC 


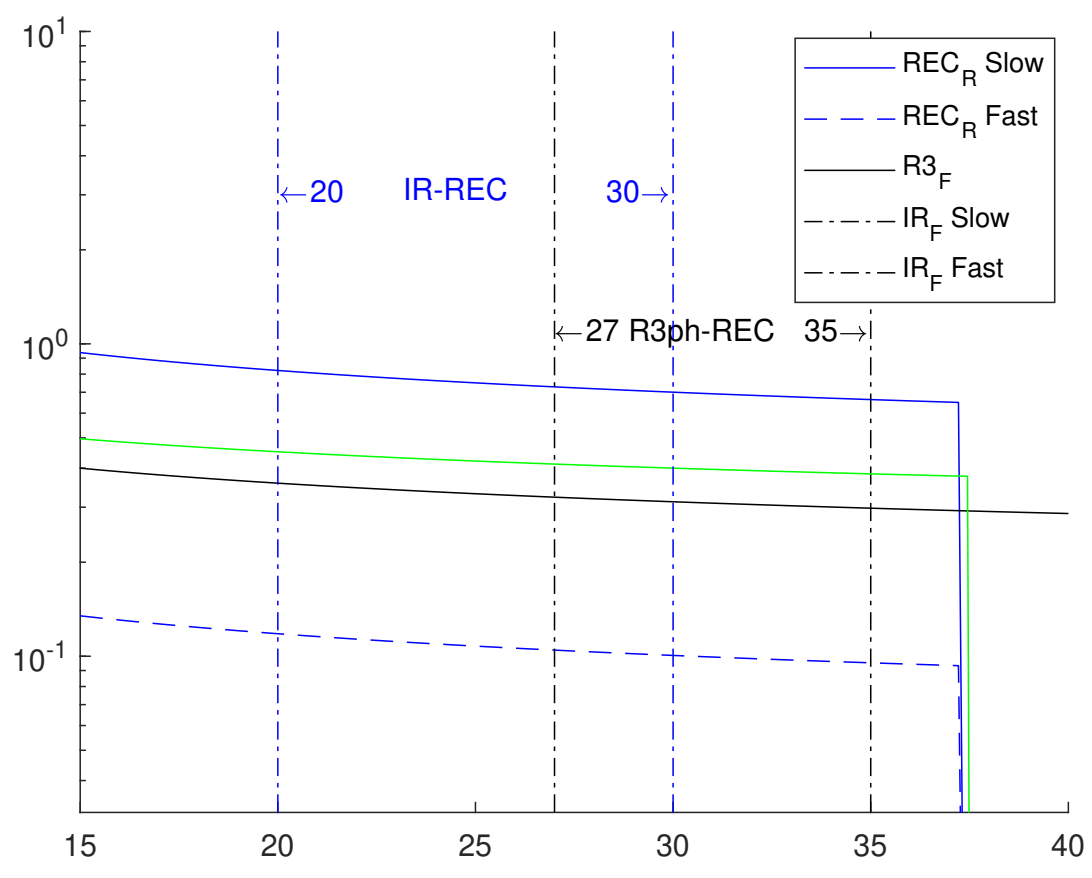

Figure 5.4: Grid connected $R E C_{R}$ TCC

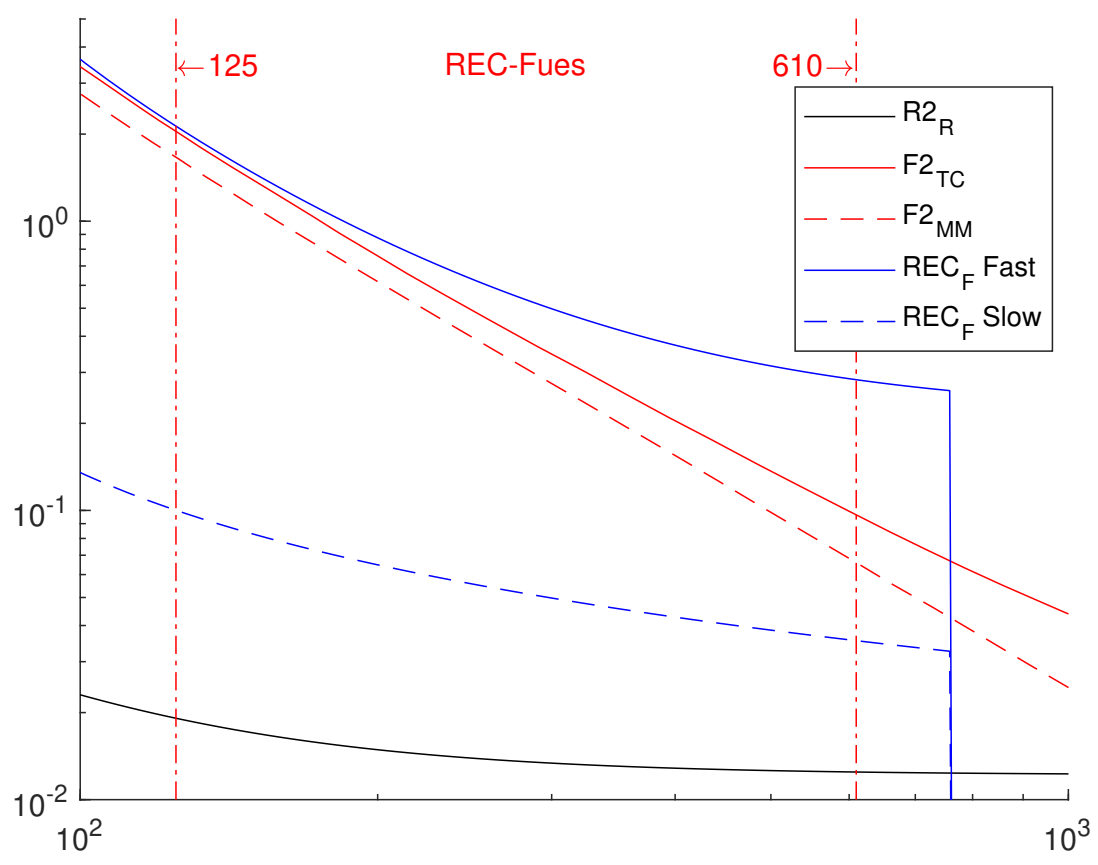

Figure 5.5: Islanded $R E C_{F}$ TCC 


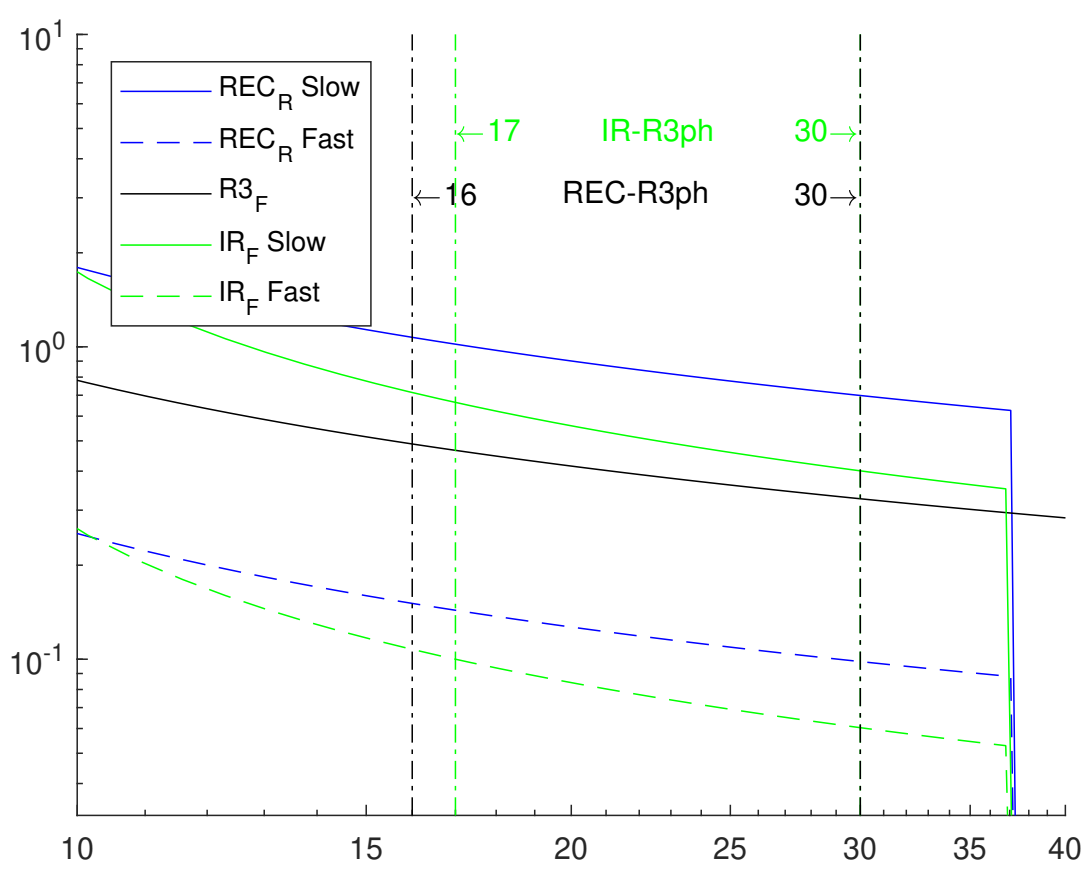

Figure 5.6: Islanded $R E C_{R}$ TCC

Table 5.1: Example settings For recloser REC at bus 5

\begin{tabular}{|c|c|c|c|c|c|}
\hline Group \# & Mode & Direction & MOC (A) & TDS & Curve \\
\hline \multicolumn{7}{|c|}{ Slow Settings } \\
\hline 1 & GC & F & 60 & 5.53 & STI \\
\hline 1 & GC & R & 5 & 7.27 & STI \\
\hline 2 & IS & F & 53 & 2.25 & VI \\
\hline 2 & IS & R & 5 & 7.25 & STI \\
\hline \multicolumn{7}{|c|}{ Fast Settings } \\
\hline 1 & GC & F & 60 & 0.5 & STI \\
\hline 1 & GC & R & 5 & 1.57 & STI \\
\hline 2 & IS & F & 53 & 0.5 & EI \\
\hline 2 & IS & R & 5 & 1.02 & STI \\
\hline
\end{tabular}




\subsection{Grid-Connected mode coordination \& settings}

In the grid-connected mode, zone 1 represents the grid, and thus any fault in zone 1 is detected by the PCC controller (using reverse setting of $R 1$ ) and the microgrid is instantaneously disconnected from the grid and switched to islanded mode with appropriate protection settings. In the grid-connected mode, for a fault inside the microgrid (zone 2-5) two scenarios can occur: the microgrid is disconnected from the grid, or the microgrid remains connected but the fault is isolated. In the case where the microgrid is disconnected from the utility grid when a fault is detected, the microgrid switches from grid-connected mode to islanded mode along with the protection. The fault is then isolated in islanded mode. In the case where the microgrid remains connected to the utility grid, it must selectively isolate the faulted part of the microgrid and remove DGs feeding that fault in order to keep the remaining part of the microgrid grid-connected. Depending on the standards and the agreement between the microgrid operator and the connected utility, either of these methods can be used.

In cases when the microgrid is islanded from the system, islanded protection will be used which is covered in section 5.3.

The scenario where the microgrid is islanded and then the fault is isolated in islanded mode is more likely. This is because the utility to which the microgrid is connected will probably not want to rely on the microgrid protection to isolate the fault from their system and likely disconnect as quickly as possible. However, as microgrids become more popular this may no longer be feasible and utilities may need to allow the microgrid to remain connected. For example, in the case of LOHO system, if the utility decides to isolate the microgrid from the system instantaneously for any fault on the microgrid, then in cases like a fault on bus 1, the utility would be unnecessarily removing the $11 \mathrm{MW}$ of generation from the system that was previously contributing a majority of its output to the utility grid. 
In grid-connected mode, for any faults in zone 2 (in LOHO system), the fault is isolated by the combined operation of $R E C, I R$, and $R 3$ on their respective reverse settings. $R E C$ is backed up by $R 2$, and $R 3$ is backed up by $R 4$. The microgrid is isolated by the forward operation of $R 1$. In this case, for the inverter-control adopted in this study, the microgrid is forced to go into a blackout as all the IBRs must be disconnected from the grid and lose the grid signal needed to operate in grid-following mode. This is unavoidable as zone 2 is the main feeder section from where the laterals originate and thus is fed by all the inverters and the grid. Therefore, during a fault, all the IBRs must be disconnected along with the grid form the microgrid in this scenario. Figure 5.7 shows the protection coordination plot for zone 2, the TCC curves are shifted to account for different percentages of the fault current seen by each device as explained in section 5.1.

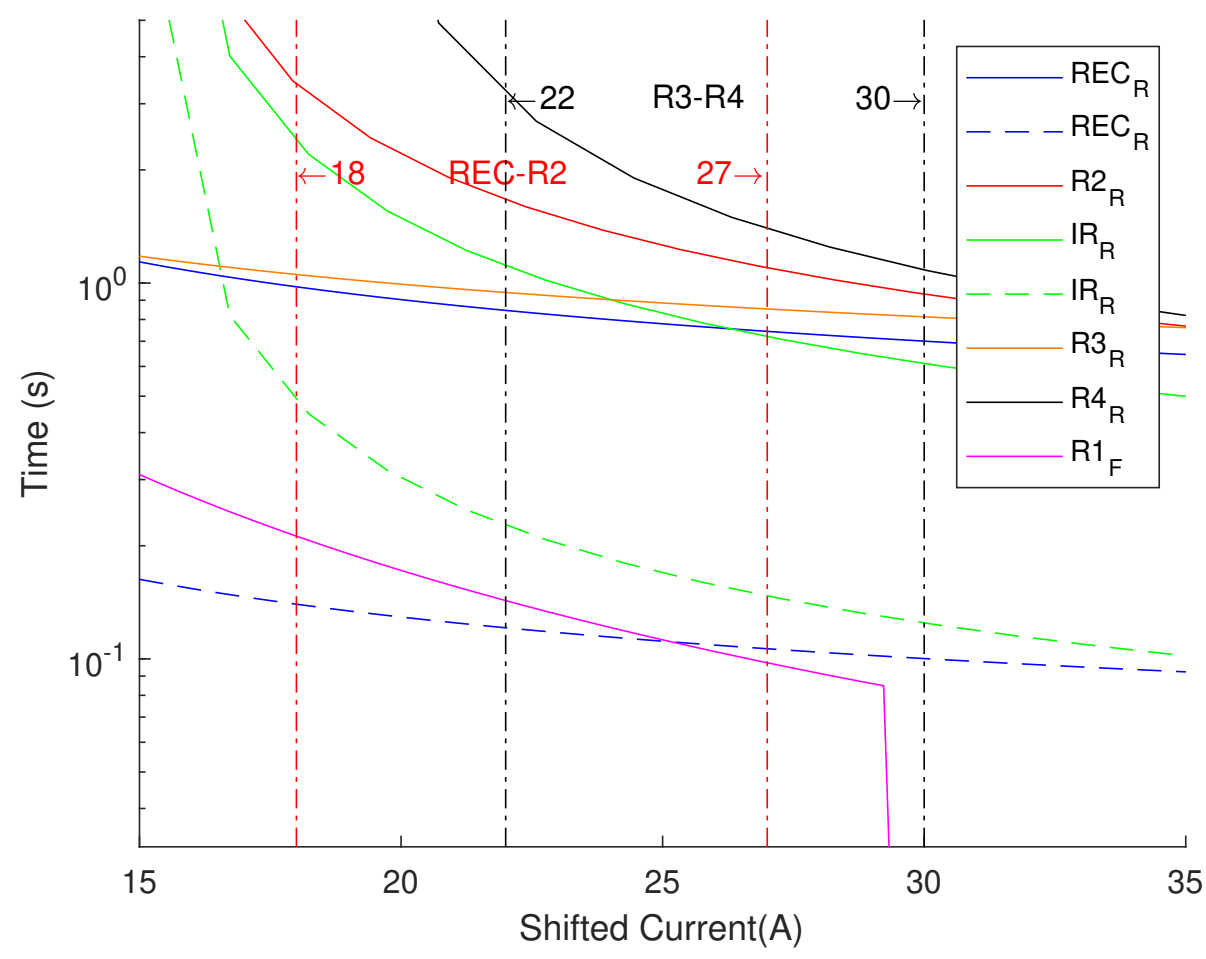

Figure 5.7: Grid connected zone 2 protection coordination 
A fault in zone 3 is isolated by the forward operation of $R E C$ followed by operation of $R 2$. $R E C$ is backed up by the reverse operation of $I R$ and $R 3$, and forward operation of $R 1$, in the event $R E C$ Fails to operate. For a fault in zone 3, only the lateral originating from Bus 5 is disconnected from the utility grid and the IBR located at bus 15 is also disconnected. However, the rest of the system is left connected to the utility grid. Figure 5.8 shows the Protection coordination plot for zone 3.

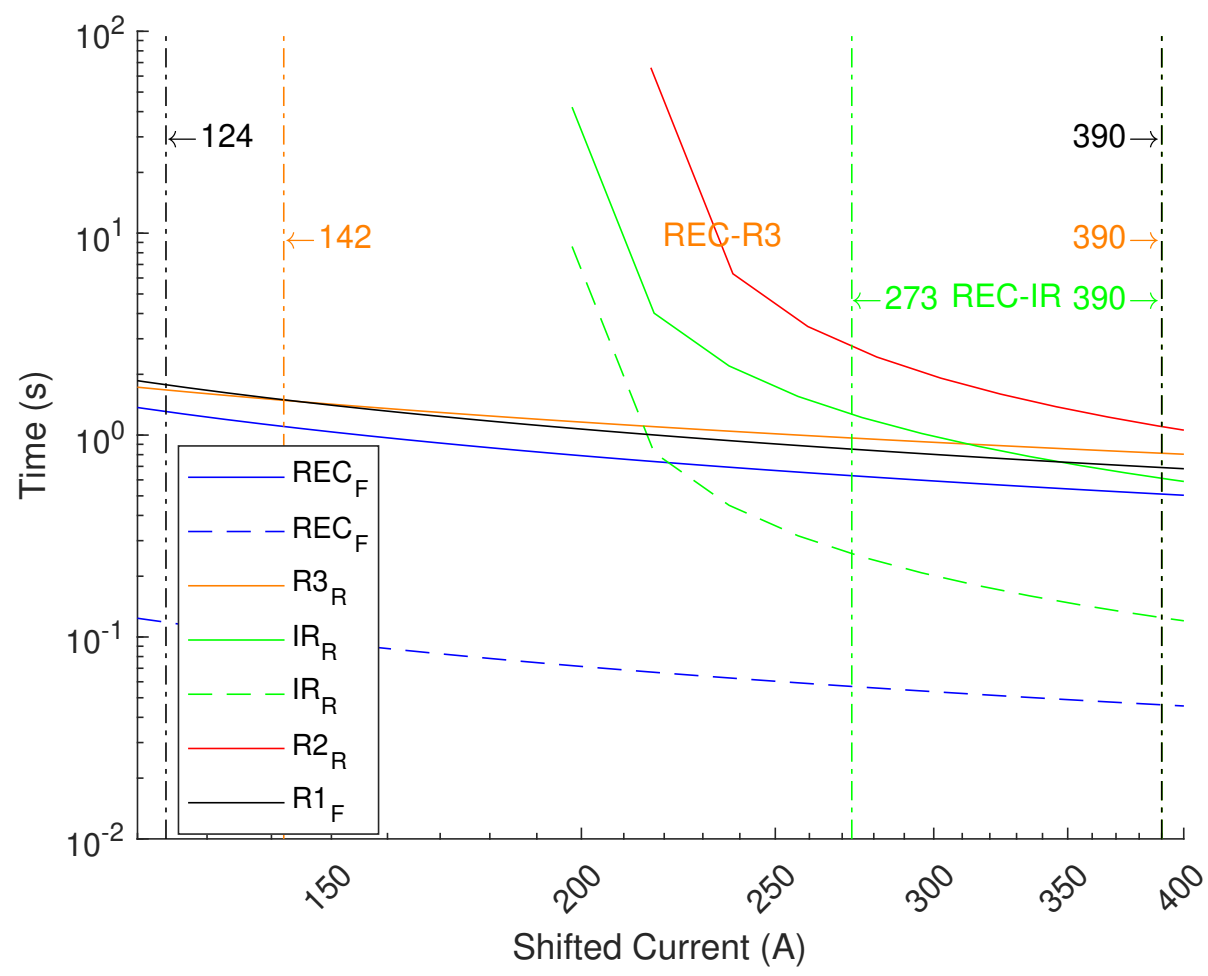

Figure 5.8: Grid connected zone 3 protection coordination

A fault in zone 4 is isolated by the forward operation of $I R$, backed up by the reverse operation of $R E C, R 3$, and the forward operation of $R 1$. For a fault in zone 4 , if the primary protection fails, then the backups must operate which creates a scenario similar to a zone 2 fault. In this case, the microgrid must be discontented from the grid and all DERs. Figure 5.9 shows the Protection coordination of zone 4.

A fault in zone 5 is isolated by the forward operation of $R 3$ and operation of $R 4$. $R 3$ 


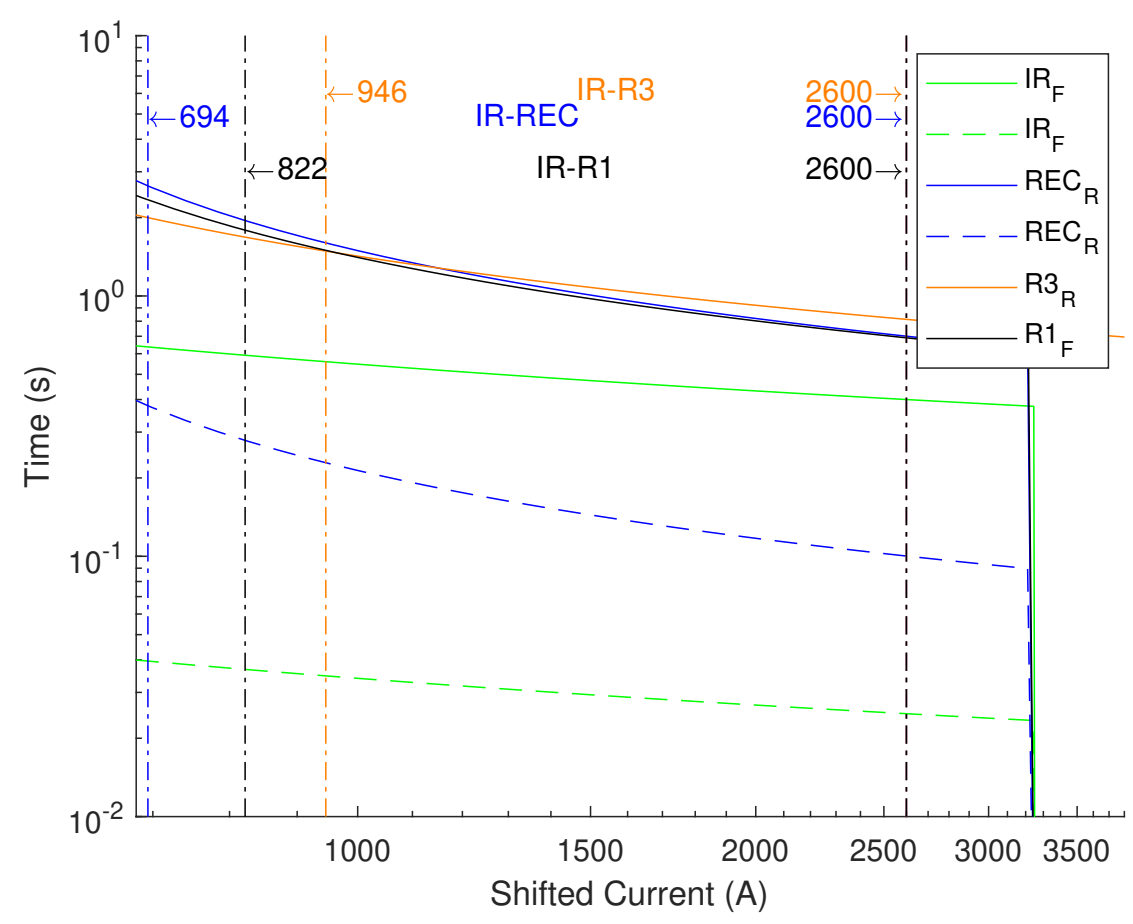

Figure 5.9: Grid connected zone 4 protection coordination

is backed up by the reverse operation of $R E C$ and $I R$ and forward direction of $R 1$. In the case of a zone 5 fault, the lateral originating at bus 8 is disconnected and the IBR on bus 12 is disconnected leaving the rest of the system to remain connected to the utility grid. Figure 5.10 shows the Protection coordination plot for zone 5.

The grid-connected mode stetting calculation was largely automated using a MATLAB script that uses the information obtained from fault analysis such as fault current range, coordination range if acting as backup, load current, etc. The calculation is done following the steps outlined in section 5.1 to calculate the protective device settings. The recloser calculation function and relay calculation function are available in the Appendix B.1 and B.2, respectively. Table 5.2 summarizes the setting calculated for the devices in grid-connected mode. Table 5.3 shows the operating times for devices for faults at each bus. 


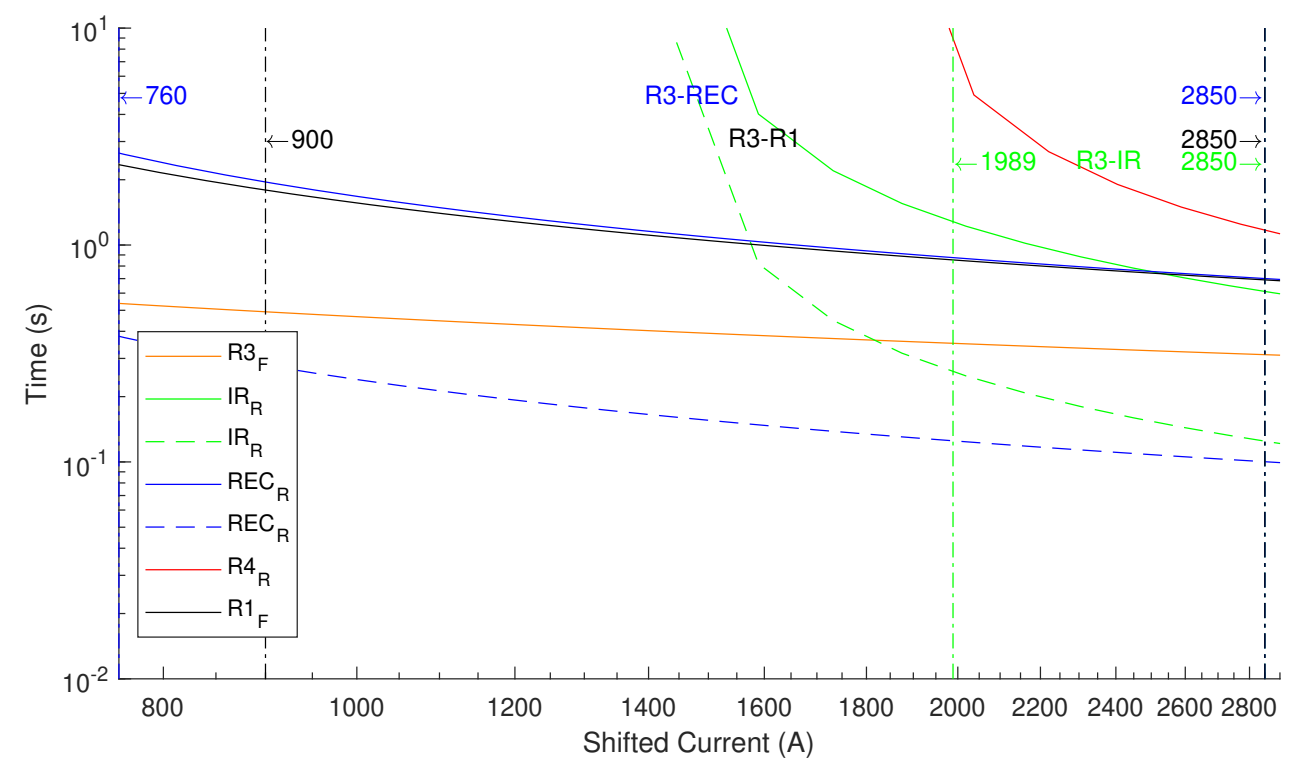

Figure 5.10: Grid connected zone 5 protection coordination

Table 5.2: Settings for protective devices in grid-connected mode

\begin{tabular}{|l|l|l|l|l|}
\hline Device & Direction & MOC (A) & TDS & Curve \\
\hline R1 & F & 440 & 7.47 & STI \\
\hline R2 & R & 14.8 & 3.84 & STI \\
\hline R2 & F & 35 & 0.5 & EI \\
\hline R3 & R & 6 & 10.41 & STI \\
\hline R3 & F & 126 & 5.60 & STI \\
\hline R4 & R & 56 & 3.00 & STI \\
\hline R4 & F & 6 & 0.50 & EI \\
\hline REC Slow & R & 5 & 7.27 & STI \\
\hline REC Fast & R & 5 & 1.04 & STI \\
\hline REC Slow & F & 60 & 5.53 & STI \\
\hline REC Fast & F & 60 & 0.50 & STI \\
\hline IR Slow & R & 432 & 2.45 & STI \\
\hline IR Fast & R & 432 & 0.50 & STI \\
\hline IR Slow & F & 78 & 8.05 & STI \\
\hline IR Fast & F & 78 & 0.50 & STI \\
\hline
\end{tabular}


Table 5.3: Operating times for a 3 ph fault during grid-connected operation ${ }^{\text {a } b}$

\begin{tabular}{|c|c|c|c|c|c|c|}
\hline $\begin{array}{l}\text { Bus } \\
\text { Zone }\end{array}$ & $\begin{array}{c}\text { Main } \\
\text { Device }\end{array}$ & $O T(s)$ & $\begin{array}{l}\text { Backup } \\
\text { Device }\end{array}$ & $O T(s)$ & $\begin{array}{c}\text { Backup } \\
\text { Device }\end{array}$ & $O T(s)$ \\
\hline $\begin{array}{c}2 \\
\mathrm{Z} 2\end{array}$ & $\begin{array}{c}R E C_{R} \\
I R_{R} \\
R 3_{R} \\
R 1_{F}\end{array}$ & $\begin{array}{c}0.16,1.11 \\
0.25,1.22 \\
1.26 \\
0.03\end{array}$ & $\begin{array}{l}R 2_{R} \\
R 4_{R}\end{array}$ & $\begin{array}{l}2.22 \\
2.24\end{array}$ & & \\
\hline $\begin{array}{l}11 \\
\mathrm{Z} 2\end{array}$ & $\begin{array}{c}R E C_{R} \\
I R_{R} \\
R 3_{R} \\
R 1_{F}\end{array}$ & $\begin{array}{c}0.16,1.11 \\
0.25,1.22 \\
1.26 \\
0.03\end{array}$ & $\begin{array}{l}R 2_{R} \\
R 4_{R}\end{array}$ & $\begin{array}{l}2.22 \\
2.23\end{array}$ & & \\
\hline $\begin{array}{c}5 \\
\mathrm{Z} 2\end{array}$ & $\begin{array}{c}R E C_{R} \\
I R_{R} \\
R 3_{R} \\
R 1_{F}\end{array}$ & $\begin{array}{c}0.16,1.11 \\
0.07,1.19 \\
1.30 \\
0.03\end{array}$ & $\begin{array}{l}R 2_{R} \\
R 4_{R}\end{array}$ & $\begin{array}{l}1.12 \\
2.04\end{array}$ & & \\
\hline $\begin{array}{c}6 \\
\mathrm{Z} 2\end{array}$ & $\begin{array}{c}R E C_{R} \\
I R_{R} \\
R 3_{R} \\
R 1_{F}\end{array}$ & $\begin{array}{c}0.15,1.02 \\
0.18,0.88 \\
0.73 \\
0.03\end{array}$ & $\begin{array}{l}R 2_{R} \\
R 4_{R}\end{array}$ & $\begin{array}{l}1.23 \\
2.24\end{array}$ & & \\
\hline $\begin{array}{l}13 \\
\mathrm{Z} 2\end{array}$ & $\begin{array}{c}R E C_{R} \\
I R_{R} \\
R 3_{R} \\
R 1_{F}\end{array}$ & $\begin{array}{c}0.15,1.04 \\
0.18,0.88 \\
0.73 \\
0.03\end{array}$ & $\begin{array}{l}R 2_{R} \\
R 4_{R}\end{array}$ & $\begin{array}{c}1.3 \\
2.24\end{array}$ & & \\
\hline $\begin{array}{c}8 \\
\mathrm{Z} 2\end{array}$ & $\begin{array}{c}R E C_{R} \\
I R_{R} \\
R 3_{R} \\
R 1_{F}\end{array}$ & $\begin{array}{c}0.16,1.09 \\
0.18,0.88 \\
0.73 \\
0.03\end{array}$ & $\begin{array}{l}R 2_{R} \\
R 4_{R}\end{array}$ & $\begin{array}{c}1.3 \\
2.25\end{array}$ & & \\
\hline $\begin{array}{c}5 \\
\mathrm{Z} 3\end{array}$ & $\begin{array}{c}R E C_{F} \\
R 2_{R}\end{array}$ & $\begin{array}{l}0.03 \\
1.23\end{array}$ & $I R_{R}$ & $0.21,1.02$ & $R 3_{R}$ & 0.77 \\
\hline $\begin{array}{c}9 \\
\mathrm{Z} 3\end{array}$ & $\begin{array}{c}R E C_{F} \\
R 2_{R}\end{array}$ & $\begin{array}{l}0.03 \\
1.23\end{array}$ & $I R_{R}$ & $0.21,1.02$ & $R 3_{R}$ & 0.77 \\
\hline $\begin{array}{l}10 \\
\mathrm{Z} 3\end{array}$ & $\begin{array}{c}R E C_{F} \\
R 2_{R}\end{array}$ & $\begin{array}{c}0.03,0.35 \\
0.69\end{array}$ & $I R_{R}$ & $0.25,1.22$ & $R 3_{R}$ & 1.29 \\
\hline $\begin{array}{l}15 \\
\mathrm{Z} 3\end{array}$ & $\begin{array}{l}F T 2 \\
R 2_{R}\end{array}$ & $\begin{array}{c}0.9 \\
0.62\end{array}$ & $R E C_{F}$ & $0.15,2.43$ & & \\
\hline $\begin{array}{l}13 \\
\mathrm{Z} 4\end{array}$ & $I R_{F}$ & 0.03 & $R E C_{R}$ & $0.15,1.04$ & $R 3_{R}$ & 0.73 \\
\hline $\begin{array}{l}14 \\
\mathrm{Z} 4\end{array}$ & $I R_{F}$ & 0.03 & $R E C_{R}$ & $0.15,1.04$ & $R 3_{R}$ & 0.73 \\
\hline $\begin{array}{c}8 \\
Z 5\end{array}$ & $\begin{array}{l}R 3_{F} \\
R 4_{R}\end{array}$ & $\begin{array}{l}0.31 \\
2.24\end{array}$ & $I R_{R}$ & $0.18,0.88$ & $R E C_{R}$ & $0.16,1.08$ \\
\hline $\begin{array}{c}7 \\
\mathrm{Z} 5\end{array}$ & $\begin{array}{l}R 3_{F} \\
R 4_{R}\end{array}$ & $\begin{array}{l}0.31 \\
2.24\end{array}$ & $I R_{R}$ & $0.18,0.88$ & $R E C_{R}$ & 0.16 .1 .08 \\
\hline $\begin{array}{c}3 \\
\mathrm{Z} 5\end{array}$ & $\begin{array}{l}R 3_{F} \\
R 4_{R}\end{array}$ & $\begin{array}{l}0.31 \\
2.24\end{array}$ & $I R_{R}$ & $0.18,0.88$ & $R E C_{R}$ & $0.16,1.08$ \\
\hline $\begin{array}{c}4 \\
\mathrm{Z} 5\end{array}$ & $\begin{array}{l}R 3_{F} \\
R 4_{R}\end{array}$ & $\begin{array}{l}0.31 \\
2.24\end{array}$ & $I R_{R}$ & $0.18,0.88$ & $R E C_{R}$ & $0.17,1.17$ \\
\hline $\begin{array}{l}12 \\
\mathrm{Z} 5\end{array}$ & $\begin{array}{l}R 3_{F} \\
R 4_{R}\end{array}$ & $\begin{array}{l}0.31 \\
2.24\end{array}$ & $I R_{R}$ & $0.18,0.88$ & $R E C_{R}$ & $0.18,1.25$ \\
\hline
\end{tabular}

${ }^{\mathrm{a}} \mathrm{F}$ - Forward Direction, R - Reverse direction.

${ }^{\mathrm{b}} \#$,\# - Represents recloser operating times for fast,slow actions. 
Overall in grid-connected mode, most faults are isolated from the grid side within $0.2 \mathrm{~s}$ with some faults taking longer, specifically faults on the low voltage side of the transformer. The transformer impedance and the current in-feed from the DER on bus 15 dramatically reduce the fault current observed to near load current levels. However, as fuse saving for the Fuse FT2 was achieved, the recloser will operate before the fuse in case of a temporary fault within $0.15 \mathrm{~s}$. For permanent faults on bus 15, it would take the Fuse FT2 0.9 s to operate. In grid-connected mode, most DERs are isolated within $1.2 \mathrm{~s}$, with only the DER on bus 12 taking up to $2.25 \mathrm{~s}$ to isolate the DER form the system. This high operating time is due to the relay $R 4_{R}$ backing up relay $R 3_{R}$ while both of them observe near-identical currents, which remain below $2 \mathrm{pu}$ thus to maintain the coordination CTI the relay $R 4_{R}$ has a larger delay before operating. It is clear that the fault clearing times are unconventionally large in some cases, especially for backup protection, but the fault currents in those cases are also unconventionally small. The fault current values in lines for such cases did not exceed 1.8 times the maximum load current values observed in the lines. This obviates the possibility of equipment-damage. The coordination scheme in grid-connected mode is capable of isolating the faults at every location in the feeder from the utility grid and the DERs present with selectivity.

\subsection{Islanded mode coordination \& settings}

In islanded mode, all fault contributions are from IBRs operating in grid-forming mode, each providing at the most $2 \mathrm{pu}$ fault current, drastically reducing the fault currents and thus requiring reductions in minimum operating currents (MOC) used by devices to detect faults in the microgrid. For example, the pickup current for $R E C$ for faults in zone 3 is $80 \mathrm{~A}$ in grid-connected mode but reduces to $53 \mathrm{~A}$ in islanded 
mode. For faults in all zones (zone 2-5), main and backup devices will remain the same as proposed for grid-connected mode, but with different settings as well as the exclusion of $R 1$. The coordination plot for zone 2 can be seen in figure 5.11 , with the device curves shifted.

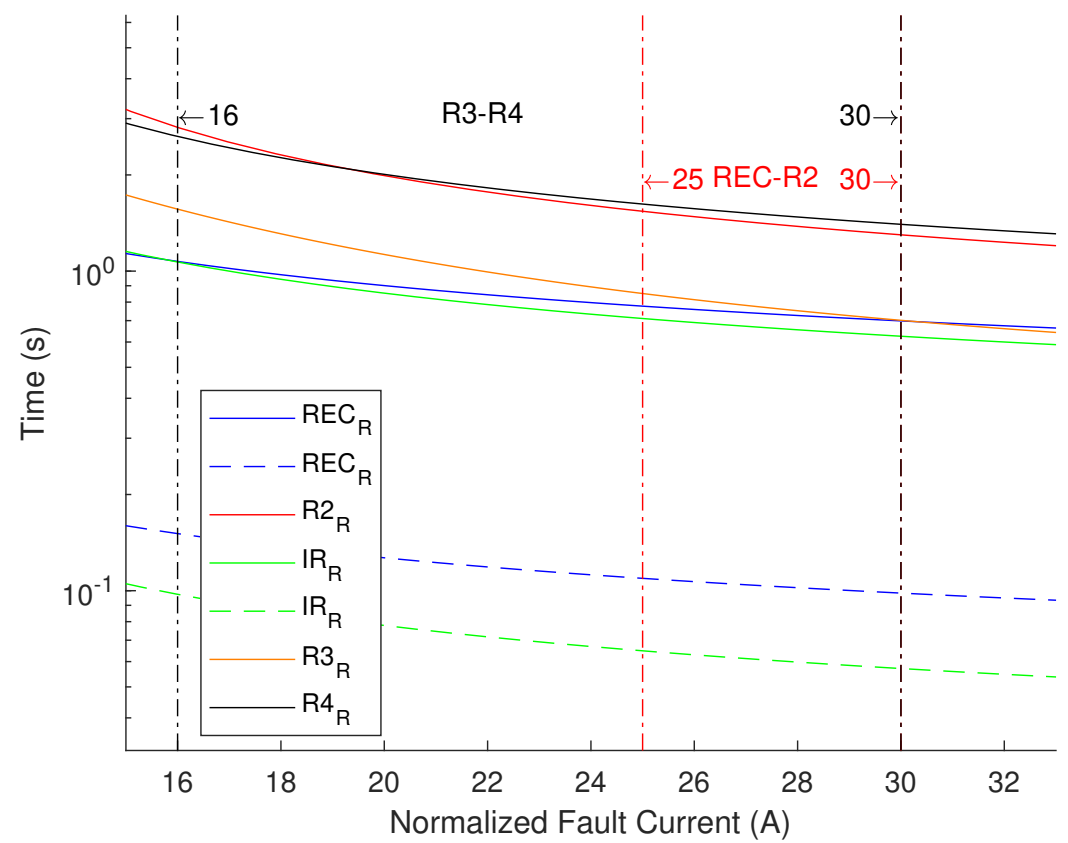

Figure 5.11: Islanded mode zone 2 protection coordination

For a fault in zone 3, the fault is isolated by $R E C_{F}$, and the DER at bus 15 is isolated by $R 2_{R}$. In the case of a zone 3 fault, the microgrid can continue to operate with the faulted section removed as the remaining part of the microgrid can still sustain itself. The coordination plot for zone 3 can be seen in figure 5.12.

For a fault in zone 4 , the fault is isolated by $I R_{F}$ which also isolates the largest DER Inverter3 in the microgrid. In this case either the microgrid needs to begin load shedding to maintain stability or reconnect to the utility grid, as the amount of distributed generation is not sufficient to supply the full load. The coordination plot for zone 3 can be seen in figure 5.13 . 


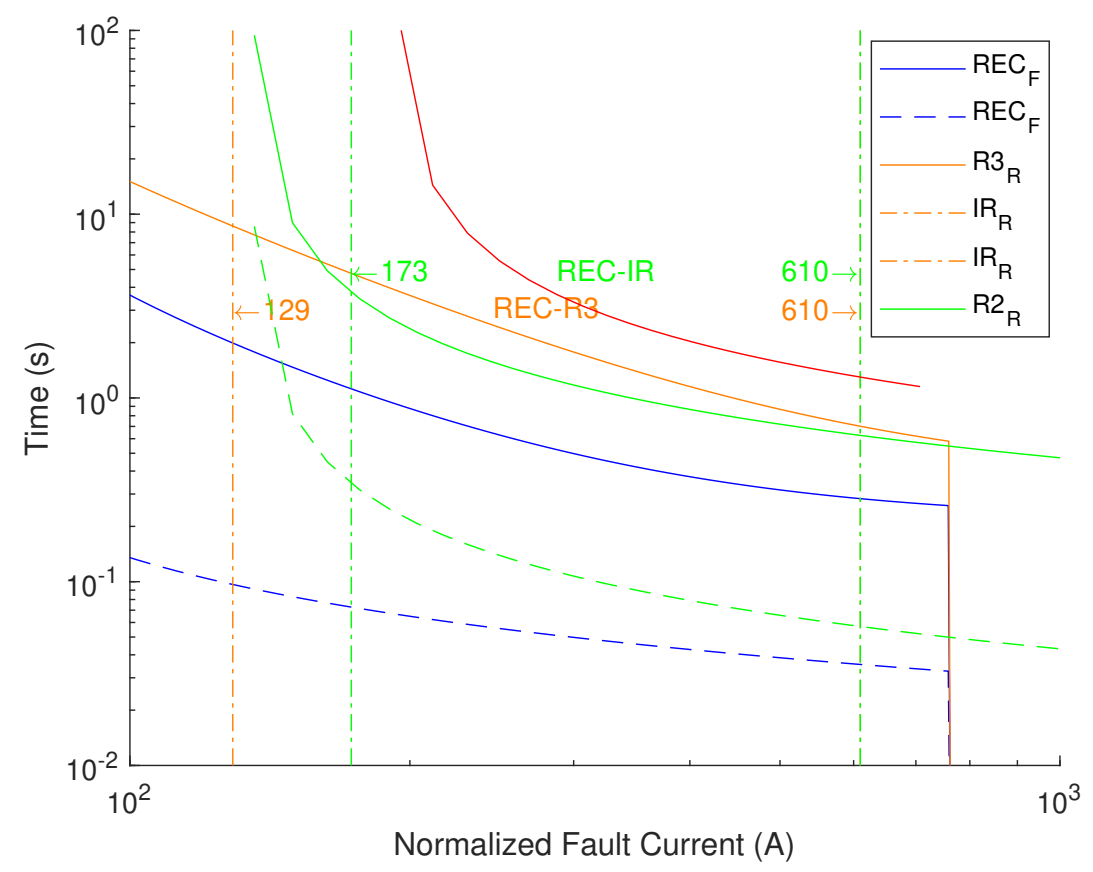

Figure 5.12: Islanded mode zone 3 protection coordination

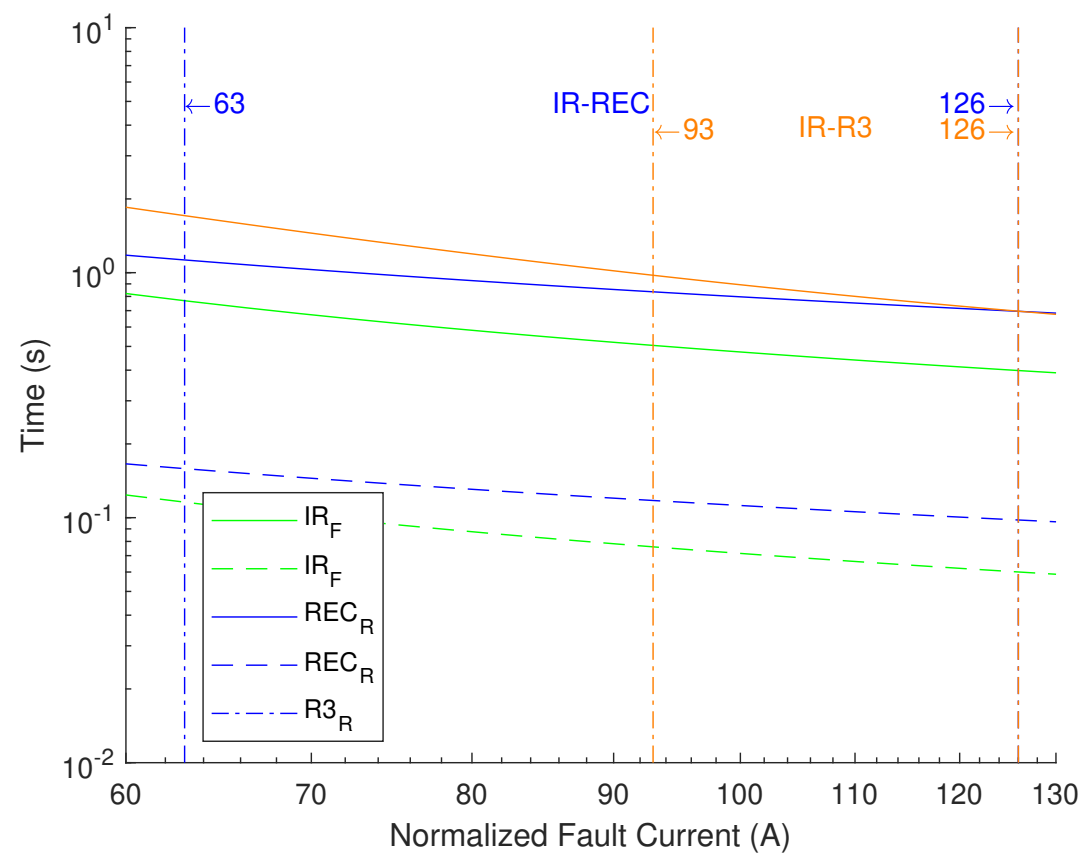

Figure 5.13: Islanded mode zone 4 protection coordination 
For a fault in zone 5 , the fault is isolated by $R 3_{F}$, and the DER at bus 12 is isolated by $R 4_{R}$. In the case of a zone 5 fault, the microgrid can continue to operate with the faulted section removed as the remaining part of the microgrid can still sustain itself. The coordination plot for zone 5 can be seen in figure 5.14.

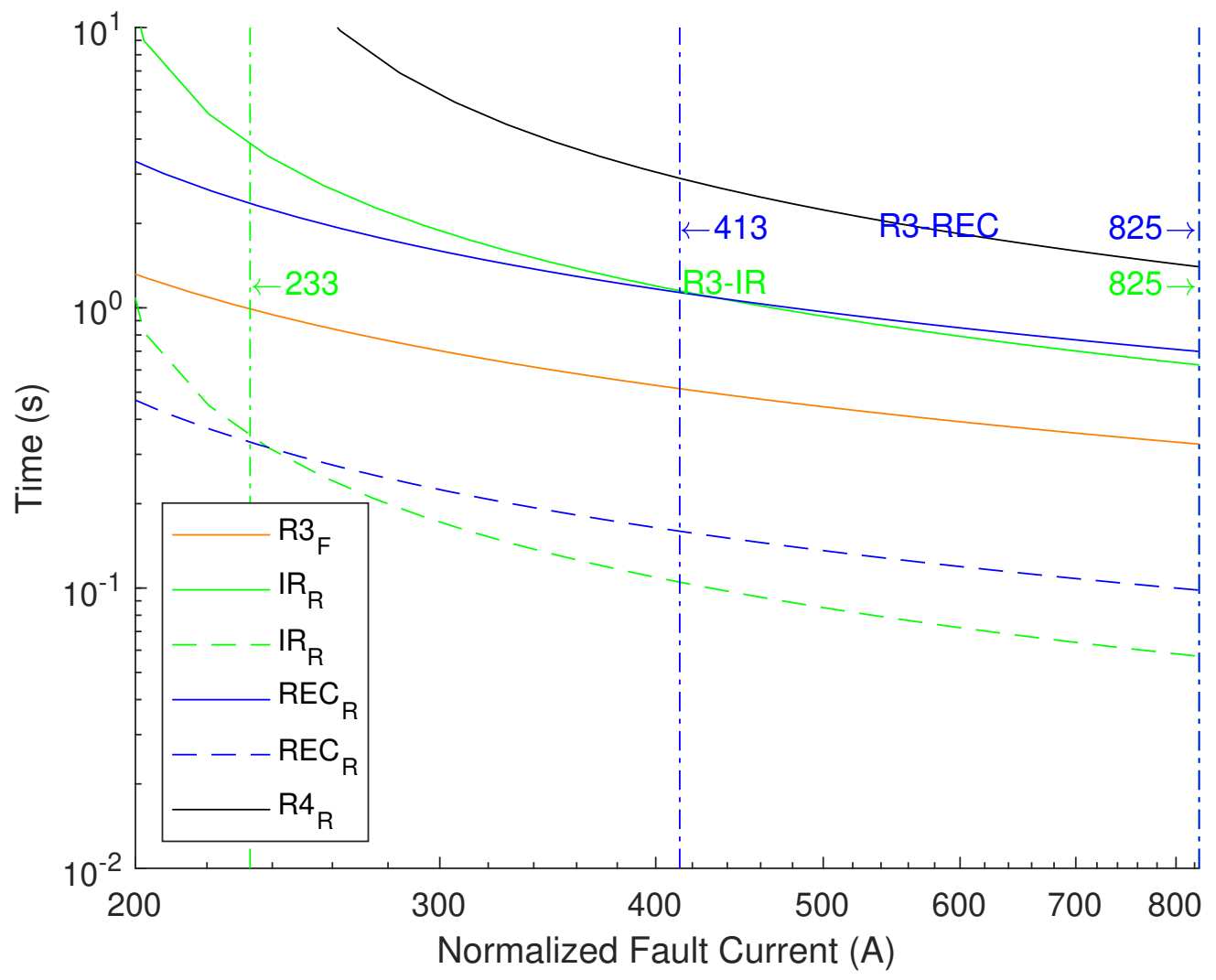

Figure 5.14: Islanded mode zone 5 protection coordination

similarly to the grid-connected mode, Islanded mode stetting calculation was largely automated using a MATLAB script that uses the information obtained from fault analysis including the fault current range and coordination range if acting as backup. The script follows the steps outlined in section 5.1 to calculate the protective device settings. The recloser calculation function and relay calculation function are available in the Appendix B.1 B.2, respectively. 
Table 5.2 summarizes the setting calculated for the devices in grid-connected mode.

Table 5.3 shows the operating times for devices for faults at each bus.

Table 5.4: Settings for protective devices in islanded mode

\begin{tabular}{|l|l|l|l|l|}
\hline Device & Direction & MOC (A) & TDS & Curve \\
\hline R2 & R & 175 & 8.78 & STI \\
\hline R2 & F & 161 & 0.50 & EI \\
\hline R3 & R & 6 & 14.95 & EI \\
\hline R3 & F & 126 & 3.55 & STI \\
\hline R4 & R & 25 & 10.86 & STI \\
\hline R4 & F & 56 & 0.50 & EI \\
\hline REC Slow & R & 5 & 7.25 & STI \\
\hline REC Fast & R & 5 & 1.02 & STI \\
\hline REC Slow & F & 53 & 2.25 & VI \\
\hline REC Fast & F & 53 & 0.50 & STI \\
\hline IR Slow & R & 180 & 5.48 & STI \\
\hline IR Fast & R & 180 & 0.50 & STI \\
\hline IR Slow & F & 30 & 3.32 & STI \\
\hline IR Fast & F & 30 & 0.50 & STI \\
\hline
\end{tabular}

Overall, in islanded mode, most faults are isolated $0.18 \mathrm{~s}$ with some faults taking longer, specifically faults on the Low voltage side of the transformer. The transformer impedance and the current in-feed from the DER on bus 15 dramatically reduce the fault current observed to near load current levels. However, as fuse saving for the Fuse FT2 was achieved, the recloser $R E C_{F}$ will operate within $0.1 \mathrm{~s}$ in case of a temporary fault. For permanent faults on bus 15, it will take the Fuse FT2 2s to operate due to the fuse having been selected based on the transformer it's protecting rather than the load current. In Islanded mode, most DERs are isolated whiten $1.2 \mathrm{~s}$, with only the DER on bus 15 taking up to $2.23 \mathrm{~s}$ to isolate the DER form the system. Similar to the grid-connected mode, the high fault clearing times, especially for backup protection, are due to less than $2 \mathrm{pu}$ fault current being supplied by the DER being isolated. The fault current values in such cases are too low to cause damage to equipment. Therefore, some longer times to isolate faults were deemed 
Table 5.5: Operating times for a $3 \mathrm{ph}$ fault at all buses during islanded operation ${ }^{\mathrm{a}, b}$

\begin{tabular}{|c|c|c|c|c|c|c|}
\hline $\begin{array}{c}\text { Bus } \\
\text { Zone }\end{array}$ & $\begin{array}{c}\text { Main } \\
\text { Device }\end{array}$ & $O T(s)$ & $\begin{array}{c}\text { Backup } \\
\text { Device }\end{array}$ & $O T(s)$ & $\begin{array}{c}\text { Backup } \\
\text { Device }\end{array}$ & $O T(s)$ \\
\hline \multirow{3}{*}{$\begin{array}{c}2 \\
\mathrm{Z} 2\end{array}$} & $R E C_{R}$ & $0.18,1.33$ & \multirow[t]{2}{*}{$R 2_{R}$} & \multirow[t]{2}{*}{1.5} & & \\
\hline & $I R_{R}$ & $0.07,1.26$ & & & & \\
\hline & $R 3_{R}$ & 1.81 & $R 4_{R}$ & 2.13 & & \\
\hline \multirow{3}{*}{$\begin{array}{l}11 \\
\mathrm{Z} 2\end{array}$} & $R E C_{R}$ & $0.17,1.24$ & \multirow[t]{2}{*}{$R 2_{R}$} & \multirow[t]{2}{*}{1.47} & & \\
\hline & $I R_{R}$ & $0.07,1.26$ & & & & \\
\hline & $R 3_{R}$ & 1.81 & $R 4_{R}$ & 2.23 & & \\
\hline 5 & $R E C_{R}$ & $0.17,1.17$ & \multirow[t]{2}{*}{$R 2_{R}$} & \multirow[t]{2}{*}{1.53} & & \\
\hline \multirow[t]{2}{*}{$\mathrm{Z} 2$} & $I R_{R}$ & $0.07,1.19$ & & & & \\
\hline & $R 3_{R}$ & 1.30 & $R 4_{R}$ & 2.04 & & \\
\hline 6 & $R E C_{R}$ & $0.17,1.17$ & \multirow[t]{2}{*}{$R 2_{R}$} & \multirow[t]{2}{*}{1.53} & & \\
\hline \multirow[t]{2}{*}{$\mathrm{Z} 2$} & $I R_{R}$ & $0.07,1.12$ & & & & \\
\hline & $R 3_{R}$ & 1.12 & $R 4_{R}$ & 1.95 & & \\
\hline 13 & $R E C_{R}$ & $0.17,1.17$ & \multirow[t]{2}{*}{$R 2_{R}$} & \multirow[t]{2}{*}{1.53} & & \\
\hline \multirow[t]{2}{*}{$\mathrm{Z} 2$} & $I R_{R}$ & $0.07,1.12$ & & & & \\
\hline & $R 3_{R}$ & 1.12 & $R 4_{R}$ & 1.95 & & \\
\hline 8 & $R E C_{R}$ & $0.17,1.17$ & \multirow[t]{2}{*}{$R 2_{R}$} & \multirow[t]{2}{*}{1.53} & & \\
\hline \multirow[t]{2}{*}{$\mathrm{Z} 2$} & $I R_{R}$ & $0.07,1.12$ & & & & \\
\hline & $R 3_{R}$ & 1.23 & $R 4_{R}$ & 1.95 & & \\
\hline $\begin{array}{c}5 \\
\mathrm{Z} 3\end{array}$ & $\begin{array}{c}R E C_{F} \\
R 2_{R}\end{array}$ & $\begin{array}{c}0.03,0.35 \\
1.53\end{array}$ & $I R_{R}$ & $0.07,1.17$ & $R 3_{R}$ & 1.30 \\
\hline $\begin{array}{c}9 \\
\mathrm{Z} 3\end{array}$ & $\begin{array}{c}R E C_{F} \\
R 2_{R}\end{array}$ & $\begin{array}{c}0.03,0.35 \\
1.53\end{array}$ & $I R_{R}$ & $0.07,1.19$ & $R 3_{R}$ & 1.29 \\
\hline 10 & $R E C_{F}$ & $0.04,0.38$ & $I R_{R}$ & $0.07,1.27$ & $R 3_{R}$ & 1.30 \\
\hline $\mathrm{Z} 3$ & $R 2_{R}$ & 1.53 & & & & \\
\hline 15 & $F T 2$ & 2.02 & $R E C_{F}$ & $0.10,2.23$ & & \\
\hline Z3 & $R 2_{R}$ & 1.73 & & & & \\
\hline $\begin{array}{l}13 \\
\mathrm{Z} 4\end{array}$ & $I R_{F}$ & $0.09,0.57$ & $R E C_{R}$ & $0.17,1.17$ & $R 3_{R}$ & 1.1225 \\
\hline $\begin{array}{l}14 \\
\mathrm{Z} 4\end{array}$ & $I R_{F}$ & $0.09,0.57$ & $R E C_{R}$ & $0.17,1.17$ & $R 3_{R}$ & 1.1225 \\
\hline 8 & $R 3_{F}$ & 0.38 & $I R_{R}$ & $0.07,1.27$ & $R E C_{R}$ & $0.17,1.17$ \\
\hline $\mathrm{Z} 5$ & $R 4_{R}$ & 1.95 & & & & \\
\hline 7 & $R 3_{F}$ & 0.38 & $I R_{R}$ & $0.07,1.12$ & $R E C_{R}$ & $0.17,1.17$ \\
\hline $\mathrm{Z} 5$ & $R 4_{R}$ & 1.95 & & & & \\
\hline 3 & $R 3_{F}$ & 0.38 & $I R_{R}$ & $0.07,1.12$ & $R E C_{R}$ & $0.17,1.17$ \\
\hline $\mathrm{Z} 5$ & $R 4_{R}$ & 1.95 & & & & \\
\hline 4 & $R 3_{F}$ & 0.38 & $I R_{R}$ & $0.07,1.19$ & $R E C_{R}$ & $0.17,1.17$ \\
\hline $\mathrm{Z} 5$ & $R 4_{R}$ & 1.95 & & & & \\
\hline 12 & $R 3_{F}$ & 0.38 & $I R_{R}$ & $0.07,1.19$ & $R E C_{R}$ & $0.17,1.17$ \\
\hline $\mathrm{Z} 5$ & $R 4_{R}$ & 1.95 & & & & \\
\hline
\end{tabular}

${ }^{\mathrm{a}} \mathrm{F}$ - Forward Direction, R - Reverse direction.

b \#,\# - Represent recloser operating times for fast,slow actions. 
acceptable. The coordination scheme in islanded mode is capable of isolating the faults at every location in the feeder from the DERs present with selectivity. However, it must be stated that in many of these cases the microgrid may not be able to sustain itself without load shedding or reconnecting the utility due to the loss of DERs. 


\section{Chapter 6}

\section{Conclusion}

The project explores the challenges faced when converting an existing real-world feeder to an IBR-fed microgrid from a control and protection perspective. A Real world $12.47 \mathrm{kV}$ distribution feeder is modeled and studied to highlight the challenges involved in protecting a feeder converted to microgrid with $100 \%$ inverter based resources. An adaptive protection solution is proposed with the constraint of using off-the-shelf equipment and minimal communication. The adaptive protection system consists for directional overcurrent relays with multiple settings which can adapt to the changing microgrid operating modes. The proposed protection scheme requires at minimum 2 setting groups, one for gird-connected mode and one for islanded mode, with individual setting for forward and reverse faults in each mode. The Directional OC relays were coordinated with each other to create a protection scheme that splits the system into 5 protection zones, each zone can be selectively removed from the microgrid, allowing for selective clearing of faults in the microgrid by removing the faulted zone form the microgrid.

Results show that the solution works well under the unusual fault profile resulting form the IBRs and the mismatch in the load and generation capacity. This study may 
be used as a guideline for other similar cases. The number of protective devices to be used and the number of zones to be created have an obvious cost-benefit trade-off. The success of the scheme also heavily depends on the fault current limits imposed by inverters and the inverter controls. Considering the extensive amount of calculation and analysis needed for this relatively small system, more complex systems are most likely to require more sophisticated solutions involving communications. 


\section{Appendix A}

\section{Appendix A: Code Used to Simulate Faults}

\section{A.1 Bash Script used to submit batch jobs to the palmetto cluster}

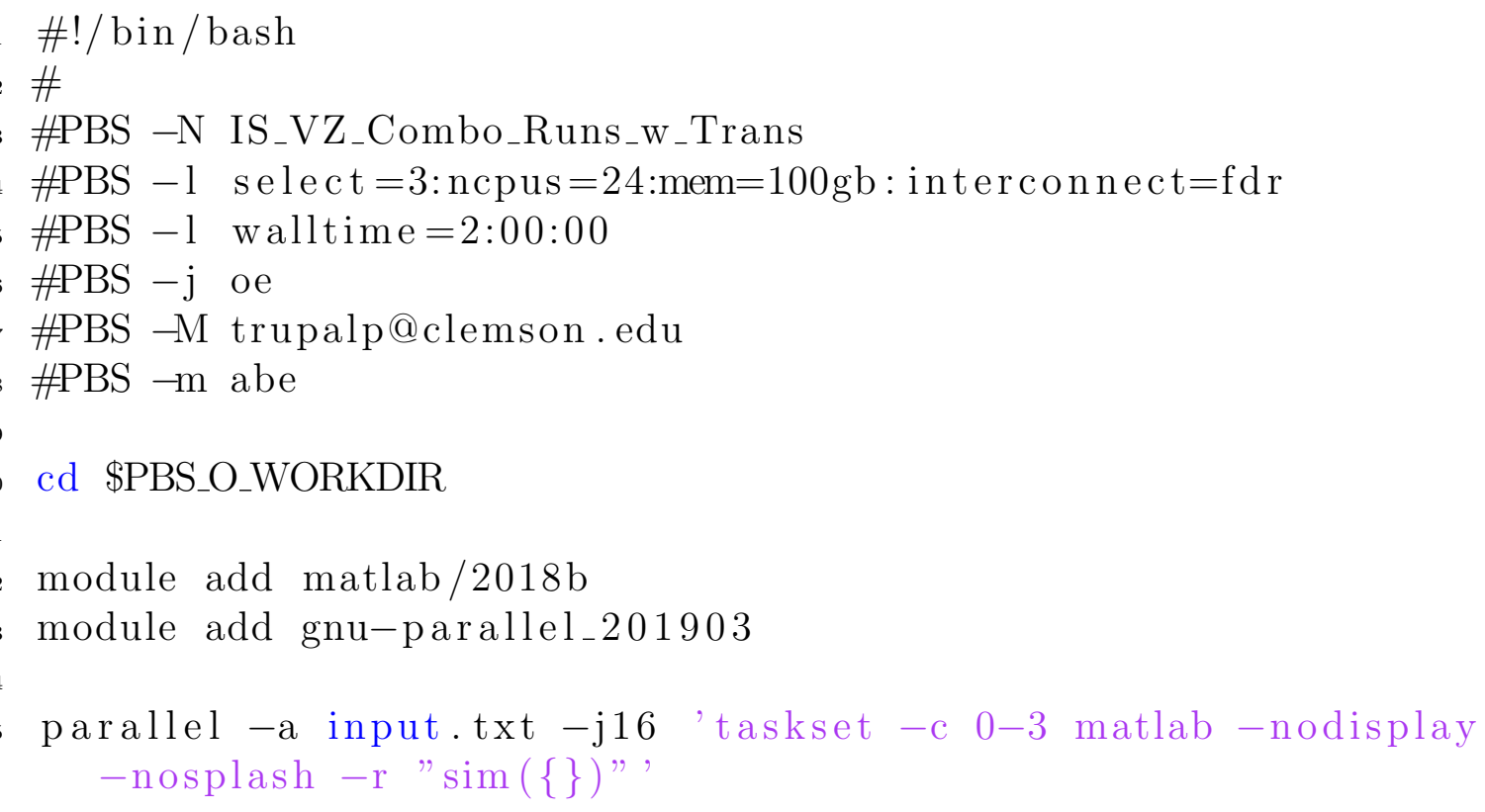




\section{A.2 Code to generate fault simulation files}

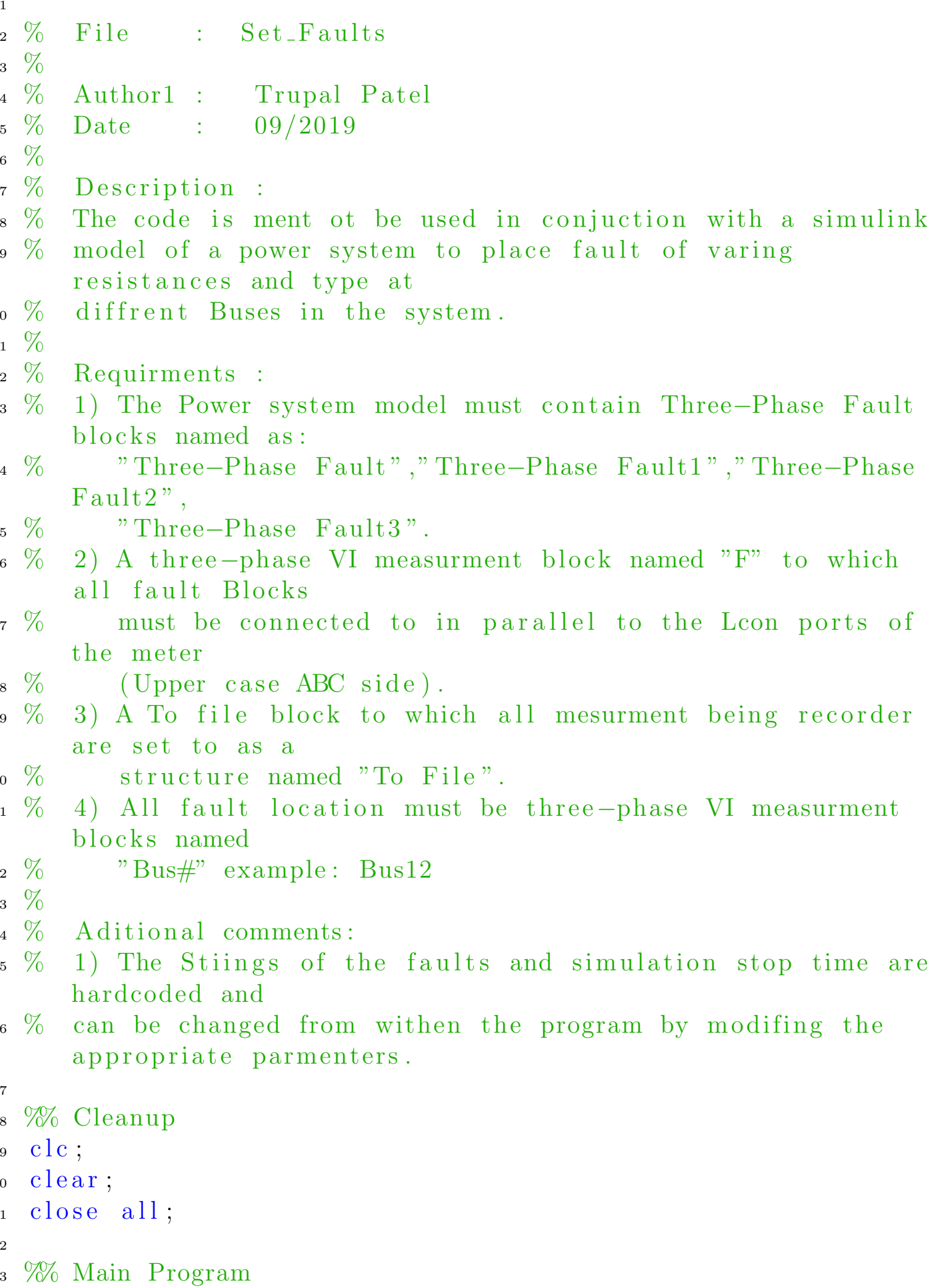




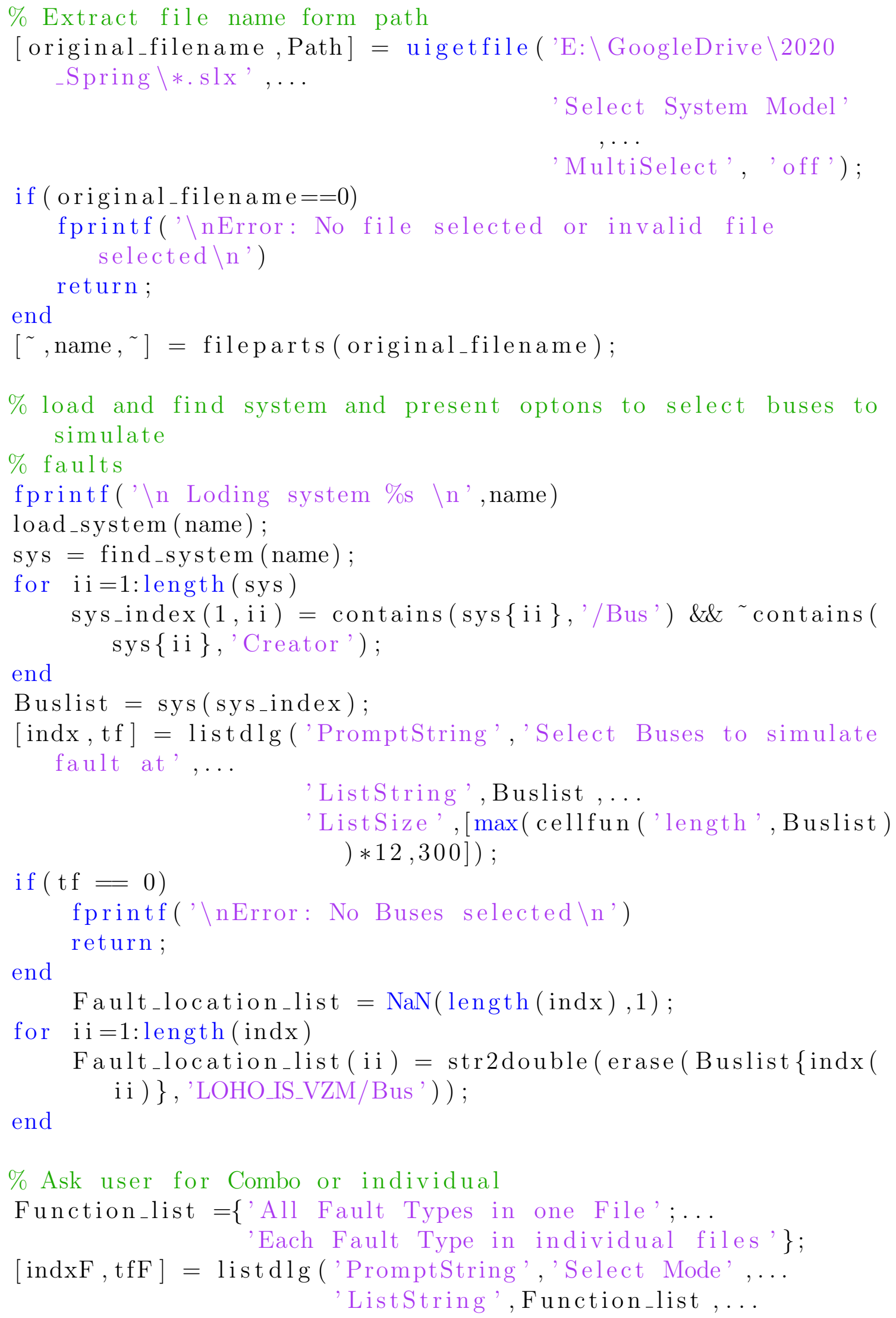

if $($ original_filename $==0)$

'Select System Model' ,$\ldots$ 'MultiSelect', 'off'); fprintf('\nError: No file selected or invalid file selected $\left.\backslash n^{\prime}\right)$

return ;

end

$\left[\sim\right.$, name,$\left.^{\sim}\right]=$ fileparts $($ original_filename $) ;$

$\%$ load and find system and present optons to select buses to simulate

$\%$ faults

fprintf('\n Loding system \%s \n', name)

load_system (name);

sys $=$ find_system (name);

for $\mathrm{i} i=1$ : length ( sys)

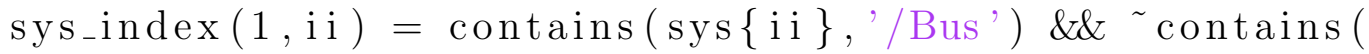
sys $\{$ ii $\}$, ' Creator');

end

Buslist $=\operatorname{sys}\left(\operatorname{sys}_{-}\right.$index $)$;

$[$ ind $x, t f]=\operatorname{listdlg}$ ('PromptString', 'Select Buses to simulate fault at', ...

if $(\mathrm{tf}=0)$

'List String', Buslist ,...

'ListSize' , $[\max ($ cellfun ('length', Buslist) )*12,300]);

fprintf('\nError: No Buses selected $\backslash n$ ') return;

end

Fault_location_list $=\operatorname{NaN}(\operatorname{length}($ indx $), 1) ;$

for $\mathrm{ii}=1$ : length (indx)

Fault_location_list ( i i ) $=$ str2double(erase( Buslist $\{$ indx (

end i i ) \}, 'LOHO_IS_VZM/Bus' ') ) ;

$\%$ Ask user for Combo or individual

Function_list $=\{$ 'All Fault Types in one File';...

'Each Fault Type in individual files' $\}$;

$[$ indxF, $\mathrm{tfF}]=\operatorname{listdlg}$ ('PromptString',' Select Mode',...

'ListString', Function_list ,... 


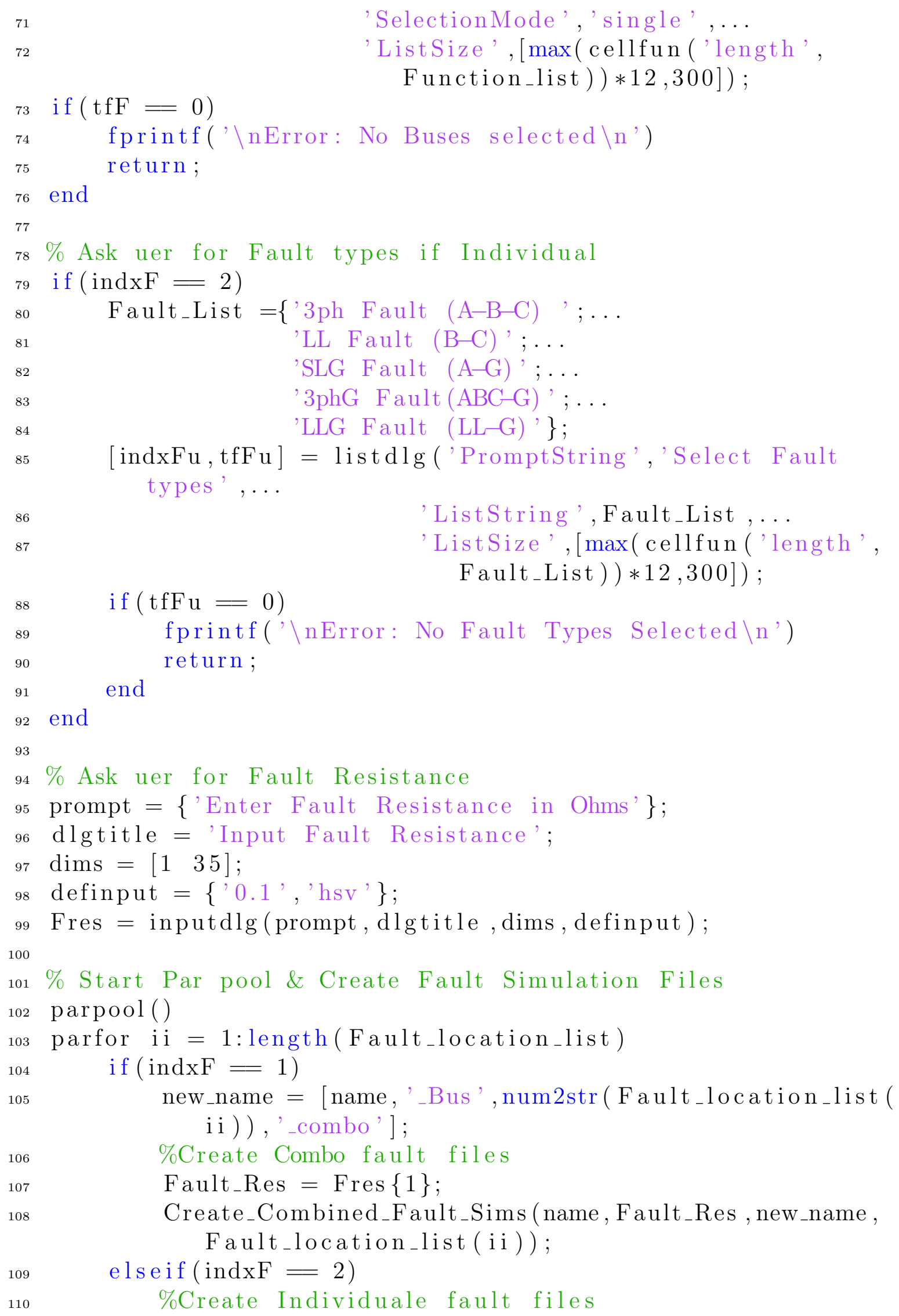




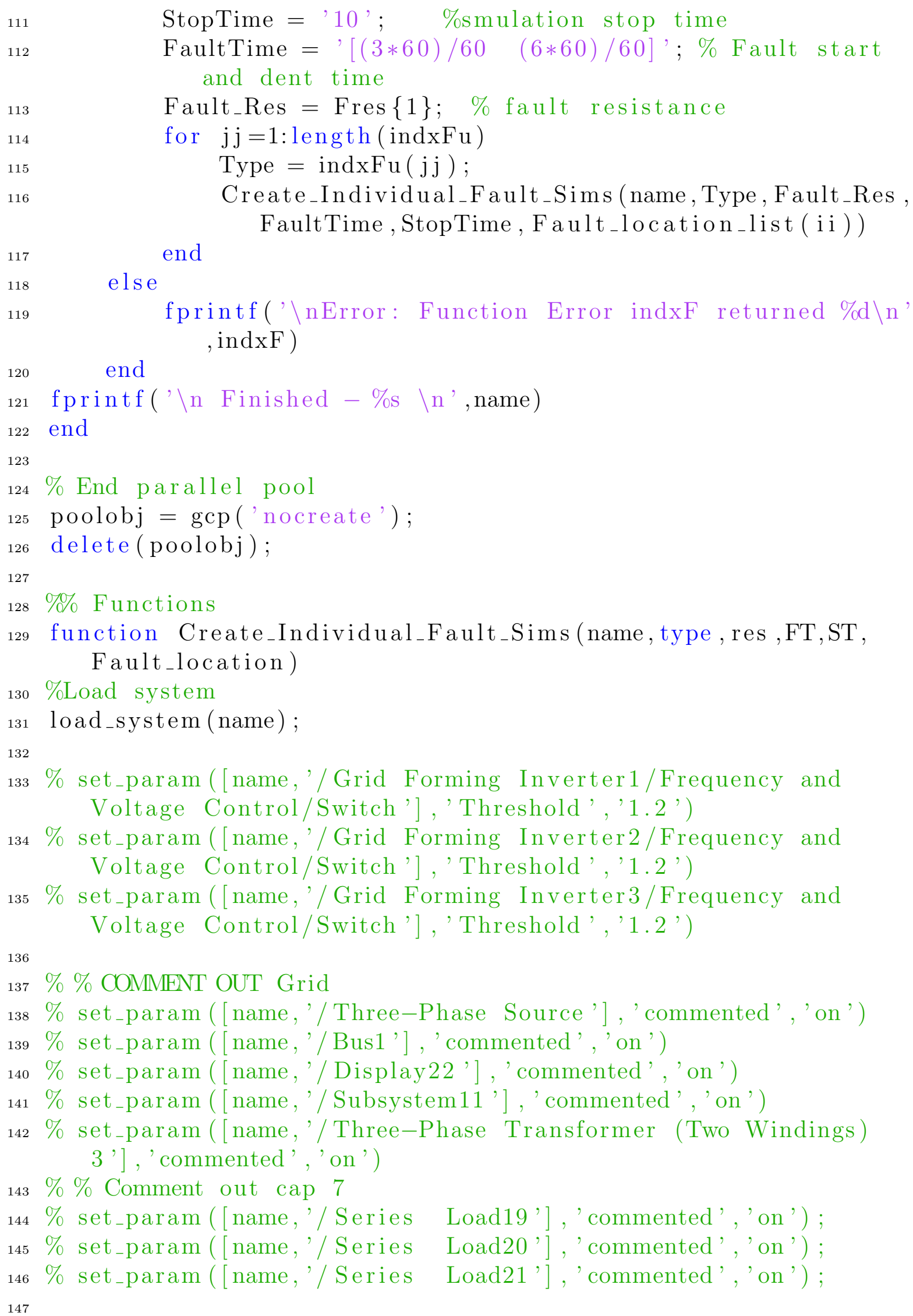


\%Connect Fault block to Correct Bus

$\mathrm{B}=$ get_param ([name, strcat ('/Bus',num2str( Fault_location $))]$, PortHandles') ;

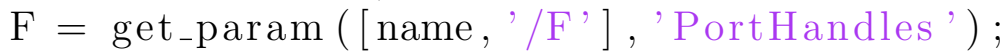

add_line (name,F.RConn,B.RConn, ' autorouting ', 'smart')

if (type $=1$ )

if exist ('ph', 'dir') $\operatorname{mkdir}($ 'ph')

end

$\%$ Comment out unnecessary faults \& set Fault timing set_param ([name, '/Three-Phase Fault'], 'commented', 'on ') set_param ([name, '/Three-Phase Fault1'], 'commented', 'on ') set_param ([name,'/Three-Phase Fault2'], 'SwitchTimes',FT) set_param ([name, '/Three-Phase Fault3'], 'commented ', 'on') set_param ([name, '/Three-Phase Fault4'], 'commented ', 'on ')

$\%$ Set fault type

set_param ([name, '/Three-Phase Fault2'], 'FaultA', 'on') set_param ([name, '/Three-Phase Fault2'], 'FaultB', 'on') set_param ([name, '/Three-Phase Fault2'], 'FaultC', 'on') set_param ([name, '/Three-Phase Fault2'], 'GroundFault', 'off ')

$\%$ Set fault reistance set_param ([name, '/Three-Phase Fault2'], 'FaultResistance', res )

$\%$ set Output File

OUT_File $=[$ erase (name, 'LOHO_' $)$, , _Bus', num $2 \operatorname{str}($

Fault_location ), '_, ', res ,'_3ph.mat'] ;

set_param ([name,'/To File'], 'Filename', OUT_File);

$\%$ Set simulation stop time

set_param (name, 'StopTime', ST)

save_system (name, ['. \ph\', name, '_Bus', num2str(

Fault_location ), '_3ph']);

\%close system if open

close_system (['.\ph\',name,'_Bus', num2str( Fault_location ) , '_3ph'],0);

elseif (type $=2$ )

if ' exist ('LL', 'dir') 


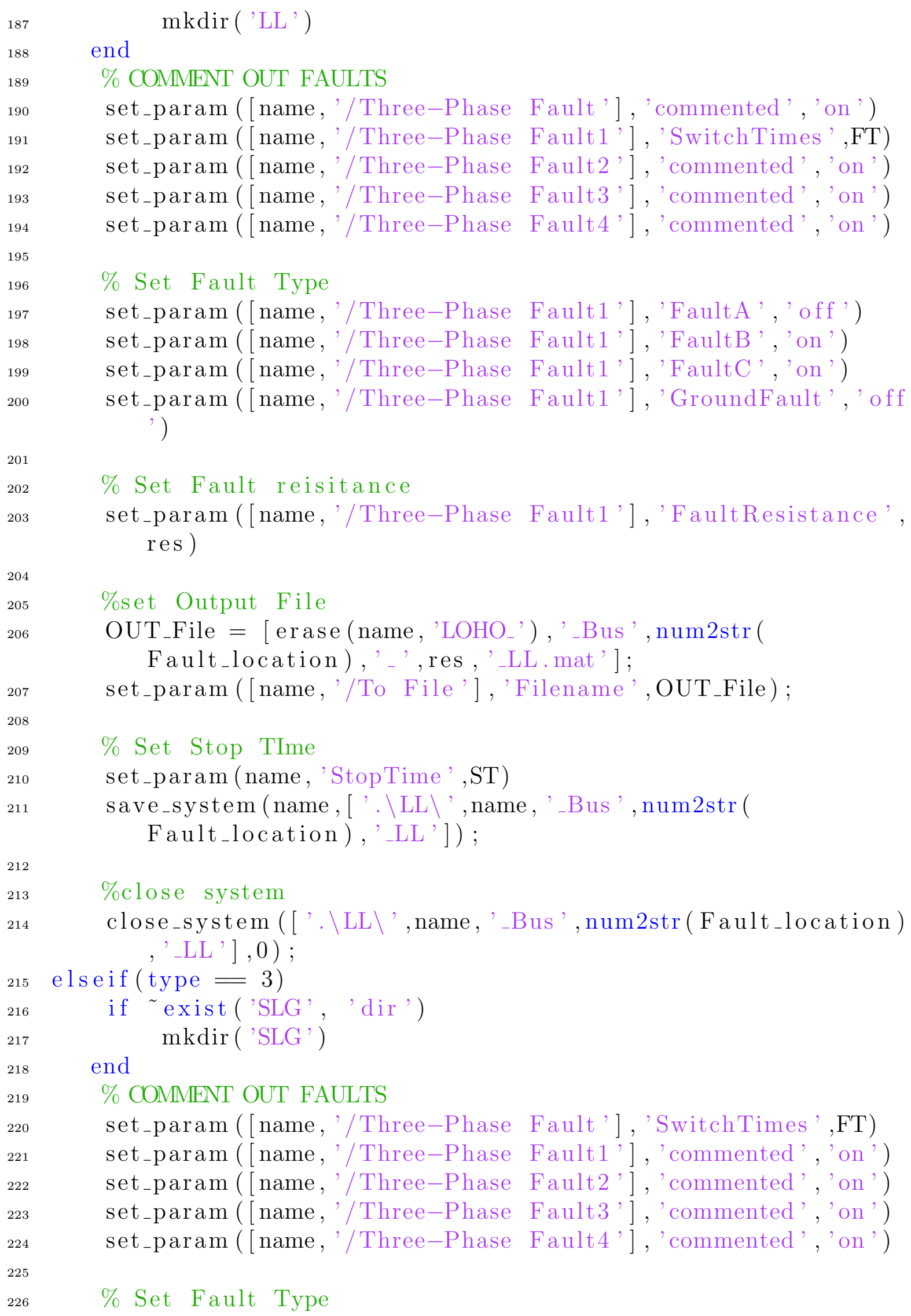

set_param ([name, '/Three-Phase Fault'], 'commented ', 'on') set_param ([name, '/Three-Phase Fault1'], 'SwitchTimes',FT)

set_param ([name, '/Three-Phase Fault2'], 'commented ', 'on ') set_param ([name, '/Three-Phase Fault3'], 'commented ', 'on ') set_param ([name, '/Three-Phase Fault4'], 'commented', 'on ')

$\%$ Set Fault Type

set_param ([name, '/Three-Phase Fault1'], 'FaultA', 'off ')

set_param ([name, '/Three-Phase Fault1'], 'FaultB', 'on')

set_param ([name, '/Three-Phase Fault1'], 'FaultC', 'on')

set_param ([name, '/Three-Phase Fault1'], 'GroundFault', 'off ')

$\%$ Set Fault reisitance

set_param([name, '/Three-Phase Fault1'], 'FaultResistance', res )

\%set Output File

OUT_File $=[$ erase (name, 'LOHO_'), '_Bus', num $2 \operatorname{str}($

Fault_location ),'_, , res, '_LL.mat' ] ;

set_param ([name,'/To File'], 'Filename',OUT_File);

$\%$ Set Stop TIme

set_param (name, 'StopTime', ST)

save_system (name, ['. \LL\', name, '_Bus', num 2 str (

Fault_location), '_LL']);

\section{\%close system}

close_system (['.\LL\', name,'_Bus', num2str( Fault_location) , '_LL'], 0$)$;

elseif (type $=3$ )

end

$\%$ COMMENT OUT FAULTS

set_param ([name, '/Three-Phase Fault'], 'SwitchTimes',FT) set_param ([name, '/Three-Phase Fault1'], 'commented ', 'on ') set_param ([name, '/Three-Phase Fault2'], 'commented ', 'on ') set_param ([name, '/Three-Phase Fault3'], 'commented ', 'on ') set_param ([name, '/Three-Phase Fault4'], 'commented', 'on ')

$\%$ Set Fault Type 
set_param ([name, '/Three-Phase Fault'], 'FaultA', 'on ') set_param ([name, '/Three-Phase Fault'], 'FaultB', 'off') set_param ([name, '/Three-Phase Fault'], 'FaultC', ' off') set_param ([name, '/ Three-Phase Fault'], 'GroundFault', 'on')

$\%$ Set Fault resistance set_param ([name, '/Three-Phase Fault'], 'FaultResistance', res )

$\%$ set Output File

OUT_File $=$ [ erase (name, 'LOHO_'), '_Bus', num $2 \operatorname{str}($

Fault_location ), '_, ', res, ',SLG.mat'] ;

set_param ([name, '/To File'], 'Filename',OUT_File);

\% Set Stop TIme

set_param (name, 'StopTime',ST)

save_system (name, ['. \SLG\', name, '_Bus', num 2 str (

Fault_location ), '_SLG']) ;

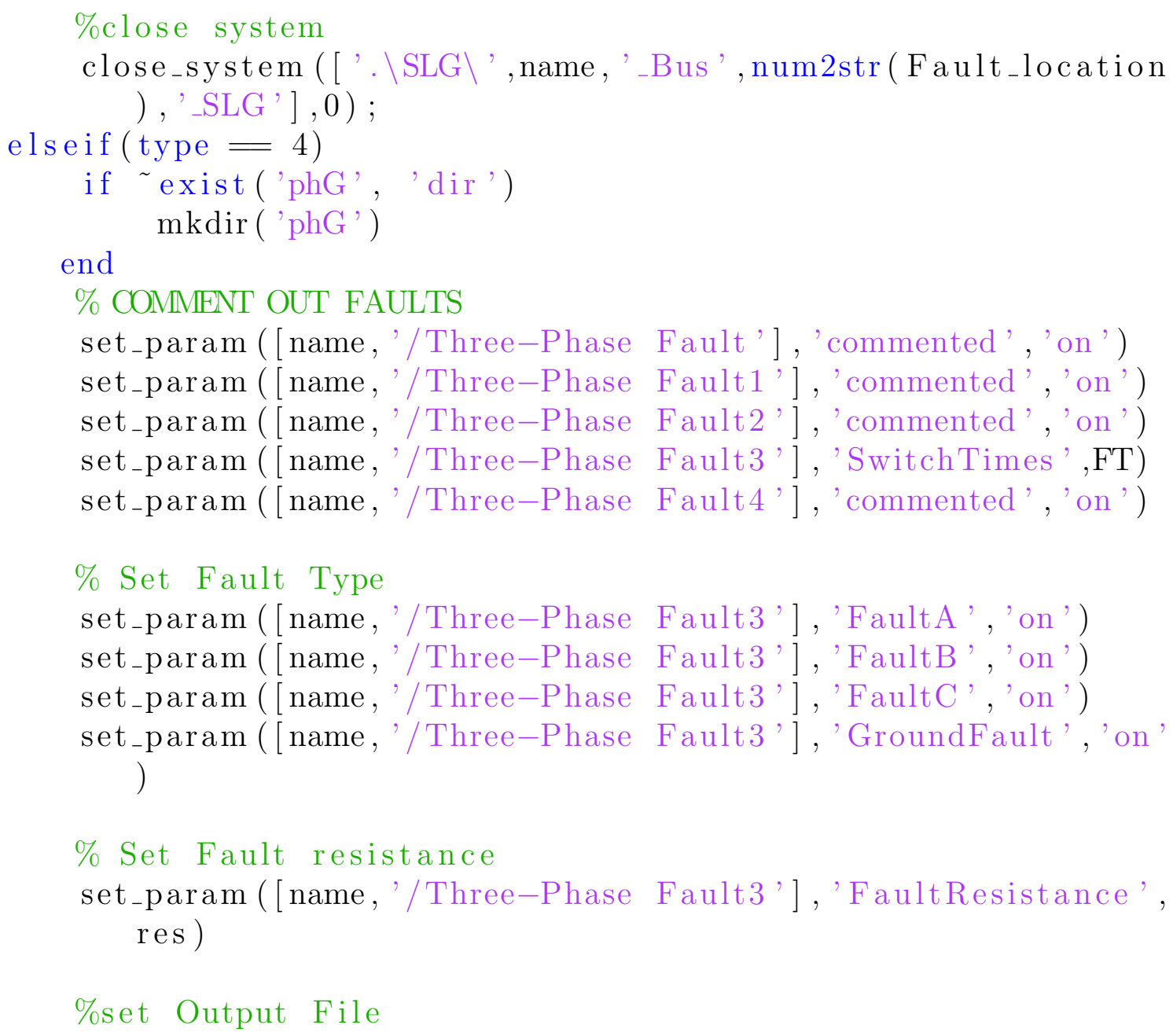

$\%$ set Output File 
OUT_File $=$ [ erase (name, 'LOHO_'), '_Bus ', num 2 str (

Fault_location ), '_, ', res ,' „phG. mat' ] ; set_param ([name,'/To File'], 'Filename',OUT_File);

\% Set Stop TIme

set_param (name, 'StopTime',ST)

save_system (name, ['. \phG\', name, '_Bus', num 2 str (

Fault_location ), '_phGF']) ;

\section{\%close system}

close_system (['. \LLG\', name,'_Bus', num2str( Fault_location

$$
\text { ), '-phGF'],0); }
$$

elseif (type $=5$ )

$$
\begin{aligned}
\text { if } ~ & \text { exist ('LLG', 'dir ') } \\
& \text { mkdir( 'LLG') }
\end{aligned}
$$

end

$\%$ OOMMENT OUT FAULTS

set_param ([name, '/Three-Phase Fault'], 'commented ', 'on ')

set_param ([name, '/Three-Phase Fault1'], 'commented', 'on ')

set_param ([name, '/Three-Phase Fault2'], 'commented ', 'on ')

set_param ([name, '/Three-Phase Fault3'], 'commented', 'on ')

set_param ([name, '/Three-Phase Fault4'], 'SwitchTimes ',FT)

$\%$ Set Fault Type

set_param ([name, '/Three-Phase Fault4'], 'FaultA', 'off ')

set_param ([name, '/Three-Phase Fault4'], 'FaultB', 'on')

set_param ([name, '/Three-Phase Fault4'], 'FaultC', 'on')

set_param ([name, '/Three-Phase Fault4'], 'GroundFault', 'on ' )

$\%$ Set Fault resistance

set_param ([name, '/Three-Phase Fault3'], 'FaultResistance', res )

\%set Output File

OUT_File $=$ [ erase (name, 'LOHO_'), '_Bus', num $2 \operatorname{str}($

Fault_location ),'_, ', res ,'_LLG.mat' ] ;

set_param ([name,'/To File'], 'Filename',OUT_File);

$\%$ Set Stop TIme

set_param (name, 'StopTime',ST)

\% Save system as new file save_system (name, ['. \LLG\', name, '_Bus', num 2 str (

Fault_location), 'LLGF']) ; 


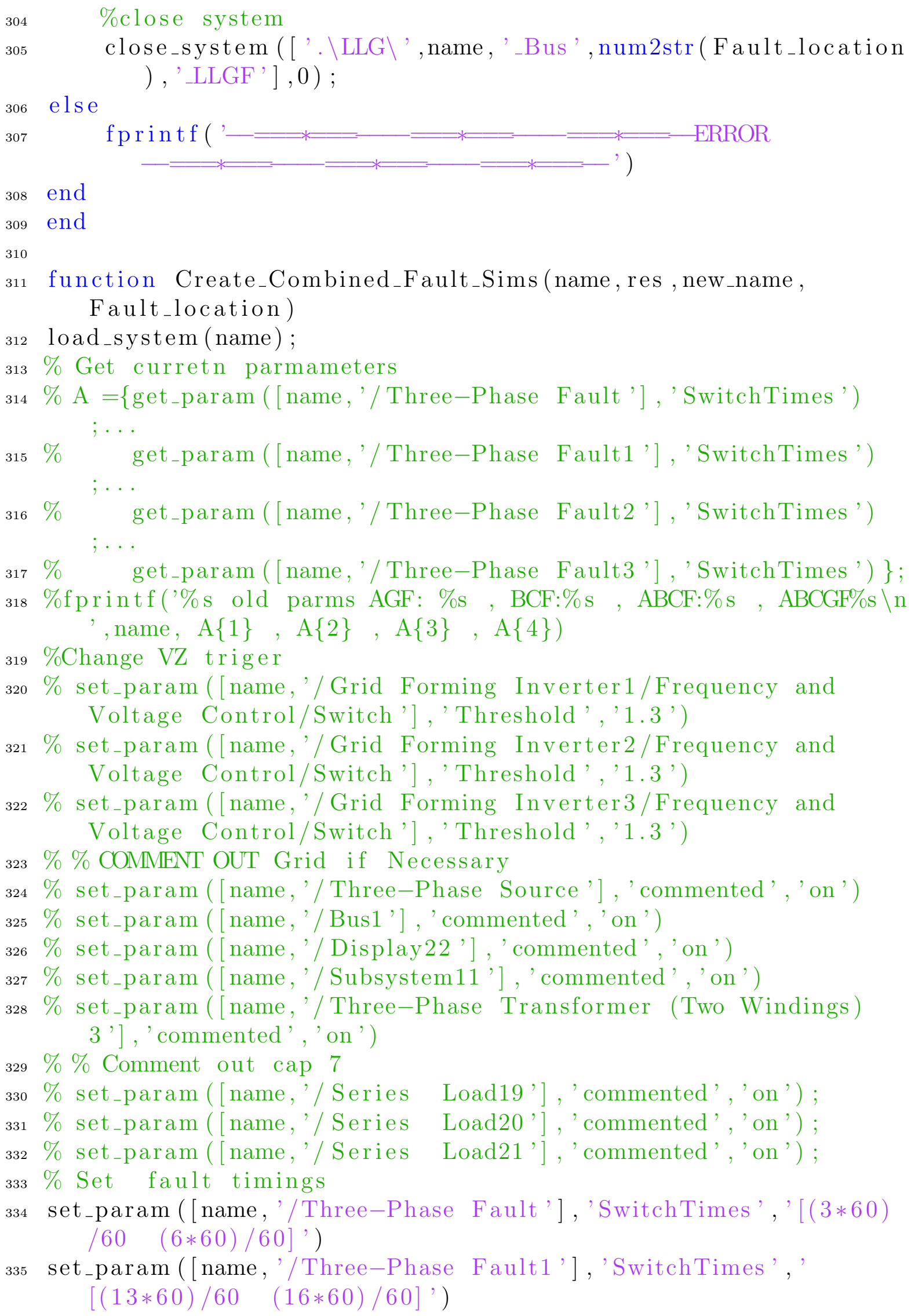

\%close system

close_system (['. \LLG \', name,'_Bus', num2str( Fault_location ), 'LLGF'],0);

else

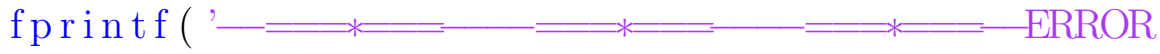

end

end

function Create_Combined_Fault_Sims (name, res, new_name, Fault_location)

load_system (name);

$\%$ Get curretn parmameters

$\% \mathrm{~A}=\{$ get_param ([name,'/ Three-Phase Fault'], 'SwitchTimes')

$; \ldots$

\% get_param ([name,'/ Three-Phase Fault1'], 'SwitchTimes') $; \ldots$

\% get_param ([name,'/ Three-Phase Fault2'], 'SwitchTimes') $; \ldots$

\% get_param ([name,' / Three-Phase Fault3'], 'SwitchTimes') \};

\%fprintf( $\%$ s old parms AGF: \%s, BCF:\%s, ABCF:\%s, ABCGF\%s $\backslash n$ , name, $\mathrm{A}\{1\}, \mathrm{A}\{2\}, \mathrm{A}\{3\}, \mathrm{A}\{4\})$

\%Change VZ triger

\% set_param ([name,'/ Grid Forming Inverter1/Frequency and

Voltage Control/Switch'], 'Threshold', '1.3')

$\%$ set_param ([name,'/ Grid Forming Inverter2/Frequency and

Voltage Control/Switch'], 'Threshold', '1.3')

$\%$ set_param ([name,'/ Grid Forming Inverter3/Frequency and

Voltage Control/Switch'], 'Threshold', '1.3')

$\% \%$ COMMENT OUT Grid if Necessary

\% set_param ([name,'/ Three-Phase Source'] , 'commented ', 'on')

$\%$ set_param ([name,'/Bus1'], 'commented', 'on ')

$\%$ set_param ([name, '/ Display22'], 'commented', 'on')

\% set_param ([name,'/ Subsystem11'], 'commented ', 'on')

\% set_param ([name,'/ Three-Phase Transformer (Two Windings)

$3^{\prime}$ '] , 'commented' , 'on')

$\%$ Comment out cap 7

$\%$ set_param ([name,' / Series Load19'], 'commented ', 'on ') ;

$\%$ set_param ([name, '/ Series Load20 '], ' commented ', 'on ') ;

\% set_param ([name,'/ Series Load21'], 'commented', 'on ') ;

$\%$ Set fault timings

set_param ([name, '/Three-Phase Fault'], 'SwitchTimes' ,' [(3*60) $\left./ 60 \quad(6 * 60) / 60]^{\prime}\right)$

set_param ([name, '/Three-Phase Fault1'], 'SwitchTimes', '

$\left.[(13 * 60) / 60 \quad(16 * 60) / 60]^{\prime}\right)$ 
set_param ([name, '/Three-Phase Fault2'], 'SwitchTimes', ' $\left.\left[\begin{array}{ll}(23 * 60) / 60 & (26 * 60) / 60\end{array}\right]^{\prime}\right)$

set_param ([name, '/Three-Phase Fault3'], 'SwitchTimes', ' $\left.[(33 * 60) / 60 \quad(36 * 60) / 60]^{\prime}\right)$ set_param ([name,'/Three-Phase Fault3'], 'SwitchTimes', ' $\left.[(43 * 60) / 60 \quad(46 * 60) / 60]^{\prime}\right)$ $\%$ Set Fault Resistance set_param ([name,'/Three-Phase Fault'], 'FaultResistance', res) set_param ([name,'/Three-Phase Fault1'], 'FaultResistance', res ) set_param ([name,'/Three-Phase Fault2'], 'FaultResistance', res) set_param ([name, '/Three-Phase Fault3'], 'FaultResistance', res ) \%Connect Fault block to Correct Bus

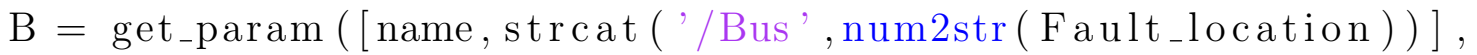
PortHandles');

$\mathrm{F}=$ get_param ([name,'/F'], 'PortHandles' $)$;

add_line (name,F.RConn,B.RConn, ' autorouting ', 'smart' )

$\%$ Set Stop Time

set_param (name, 'StopTime', ' 50 ')

$\%$ Set Output File

OUT_File $=$ [ erase (new_name, 'LOHO_' $)$,' _, ', res , '. mat' ] ;

set_param ([name,'/To File'], 'Filename',OUT_File);

$\%$ Save as copy

if 'exist ('Combo', 'dir')

mkdir ('Combo')

end

save_system (name, [' \ \Combo\', new_name ]) ;

close_system ( [ '.\Combo\', new_name $], 0)$;

fprintf( '\n Created \%s \n', new_name)

end 


\section{Appendix B}

\section{Appendix B: Code to Calculate Device Settings}

\section{B.1 Function to calculate recloser settings}

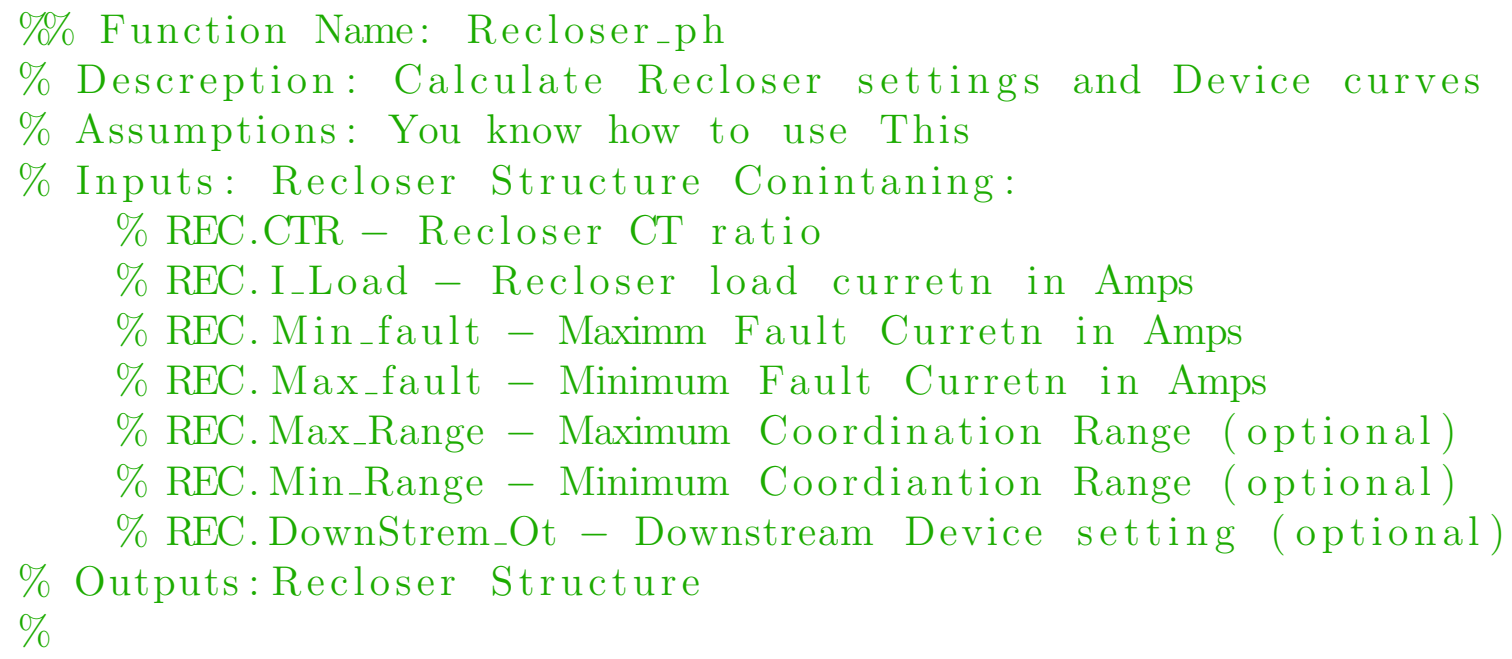




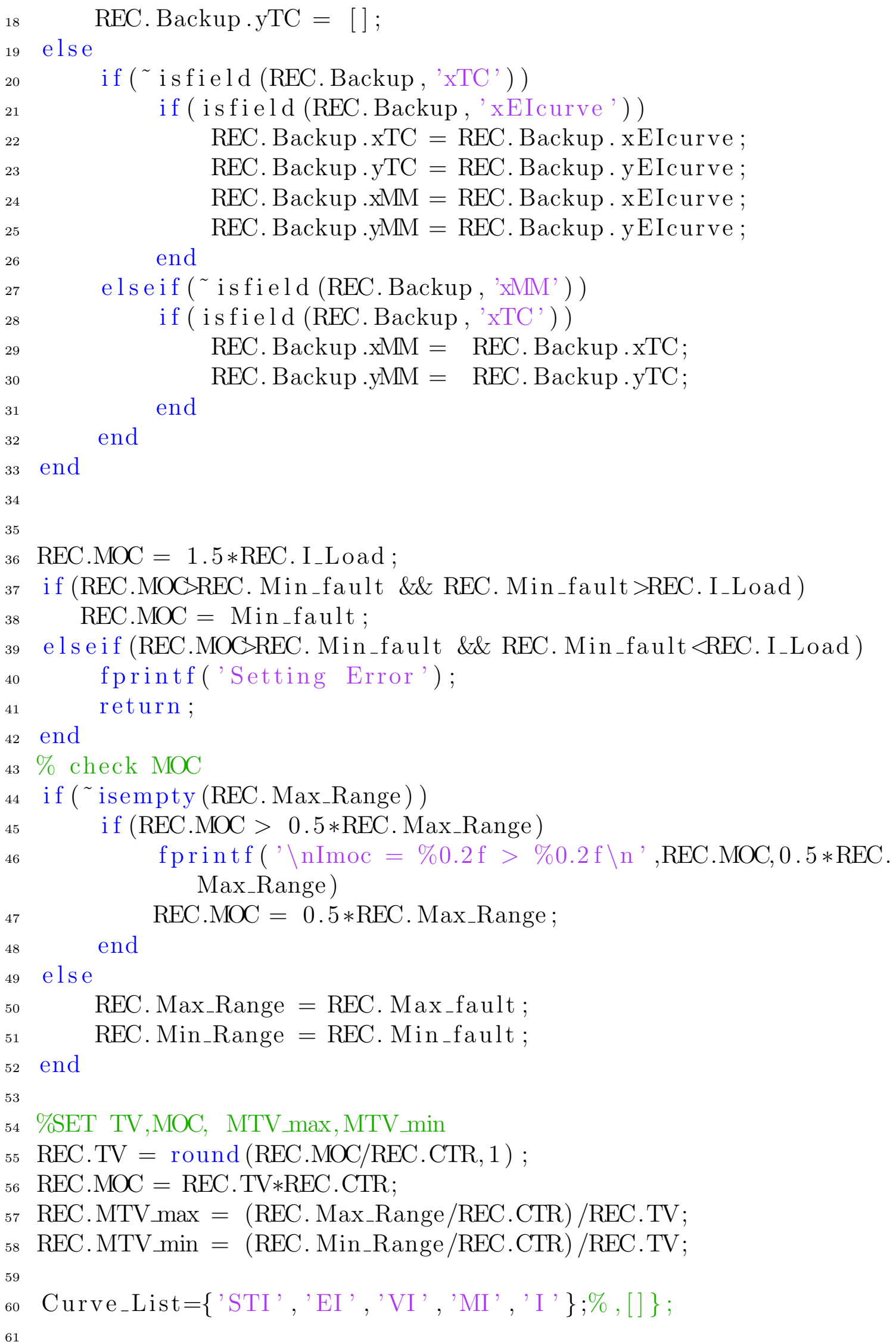




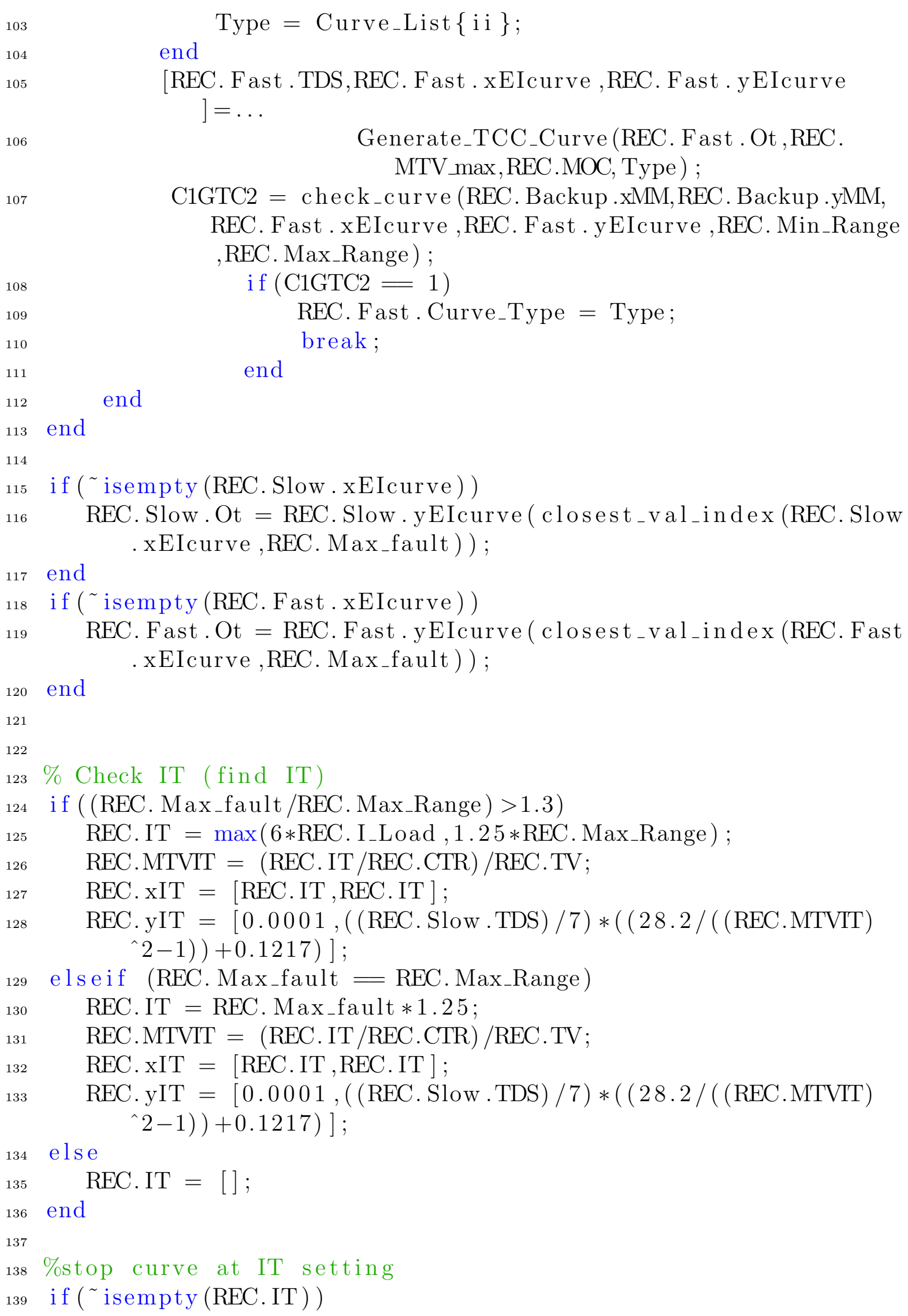

end

Type $=$ Curve_List $\{\mathrm{i} i\}$;

[REC. Fast . TDS, REC. Fast . xEIcurve ,REC. Fast . yEIcurve ]$=\ldots$

Generate_TCC_Curve(REC. Fast . Ot, REC. MTV_max, REC.MOC, Type) ;

C1GTC2 = check_curve (REC. Backup.xMM,REC. Backup.yMM, REC. Fast . xEIcurve ,REC. Fast . yEIcurve ,REC. Min_Range ,REC. Max_Range) ;

$$
\text { if ( } 1 \text { GTC2 }=1)
$$

REC. Fast. Curve_Type = Type;

break;

end

end

end

if ( isempty (REC. Slow . xEIcurve))

REC. Slow. Ot $=$ REC. Slow $\cdot$ yEIcurve ( closest_val_index (REC. Slow

end .xEIcurve,REC. Max_fault));

if ( isempty (REC. Fast.xEIcurve))

REC. Fast. Ot = REC. Fast.yEIcurve (closest_val_index (REC. Fast .xEIcurve,REC. Max_fault)) ;

end

\section{$\%$ Check IT (find IT)}

if ( (REC. Max_fault/REC. Max_Range $)>1.3)$

REC.IT $=\max (6 *$ REC.I_Load , $1.25 *$ REC.Max_Range $)$;

REC.MTVIT $=($ REC. IT $/$ REC.CTR $) /$ REC.TV;

REC.xIT $=[$ REC.IT, REC.IT $]$;

REC.yIT $=[0.0001,(($ REC. Slow. TDS $) / 7) *((28.2 /(($ REC.MTVIT $)$ $2-1))+0.1217)]$;

elseif (REC.Max_fault $=$ REC.Max_Range)

REC. IT $=$ REC. Max_fault $* 1.25$;

REC. MTVIT $=($ REC. IT $/$ REC.CTR $) /$ REC.TV;

REC. $x I T=[$ REC.IT, REC. IT $]$;

REC.yIT $=[0.0001,(($ REC. Slow. TDS $) / 7) *((28.2 /(($ REC.MTVIT $)$

$$
2-1))+0.1217)]
$$

els e

REC.IT $=[] ;$

end

\%stop curve at IT setting

if ( isempty (REC.IT)) 


\section{B.2 Function to calculate relay settings}

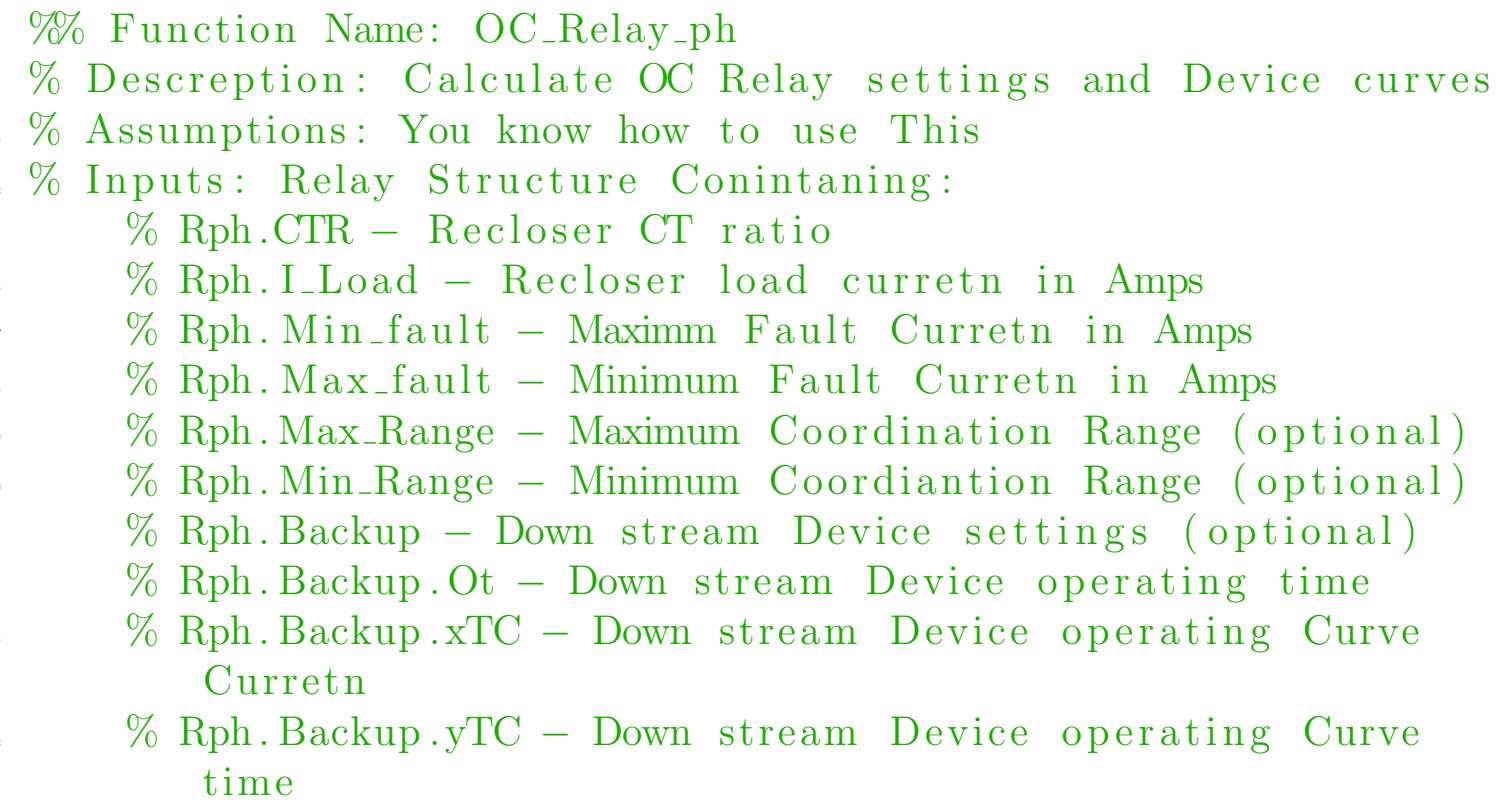




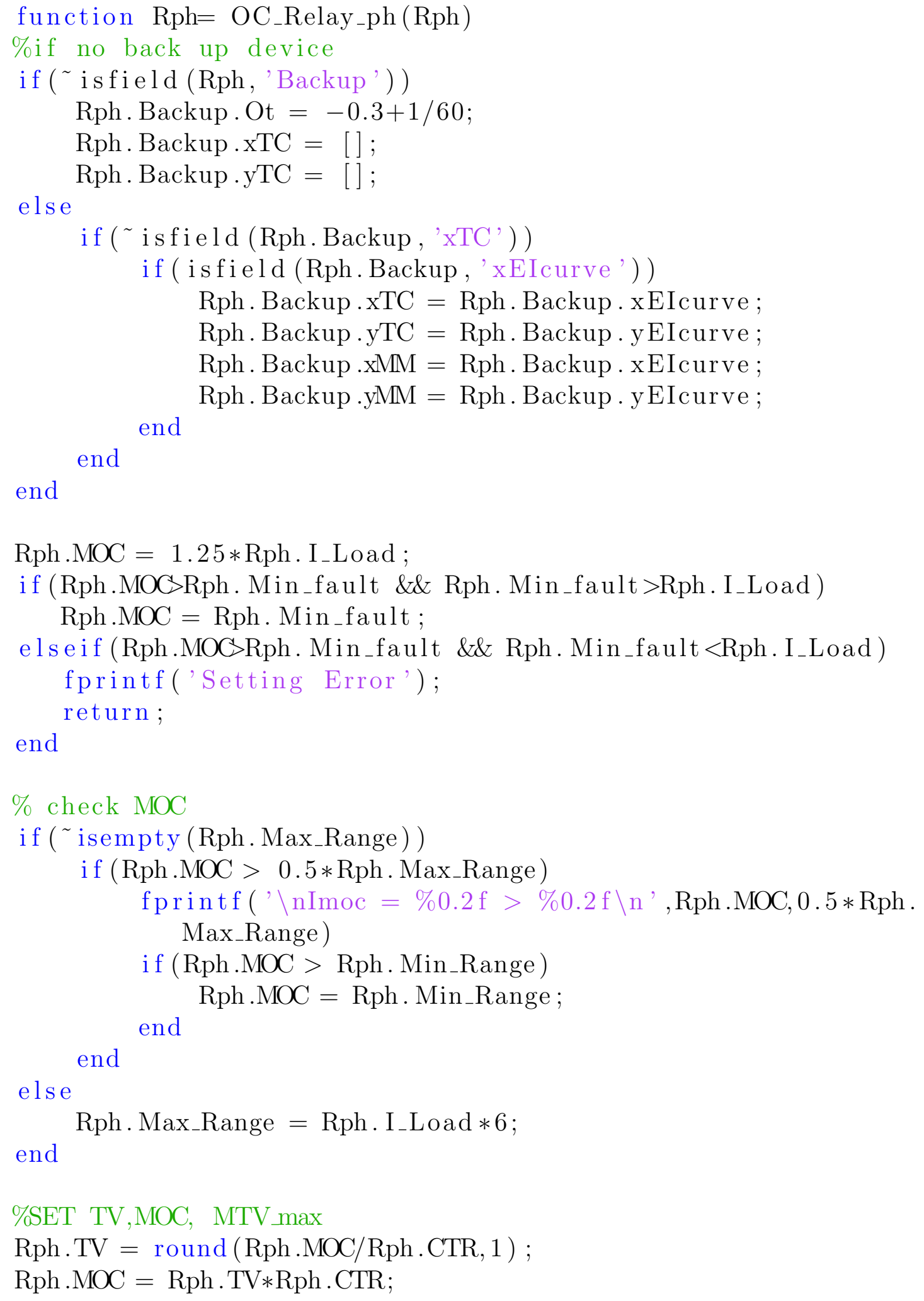




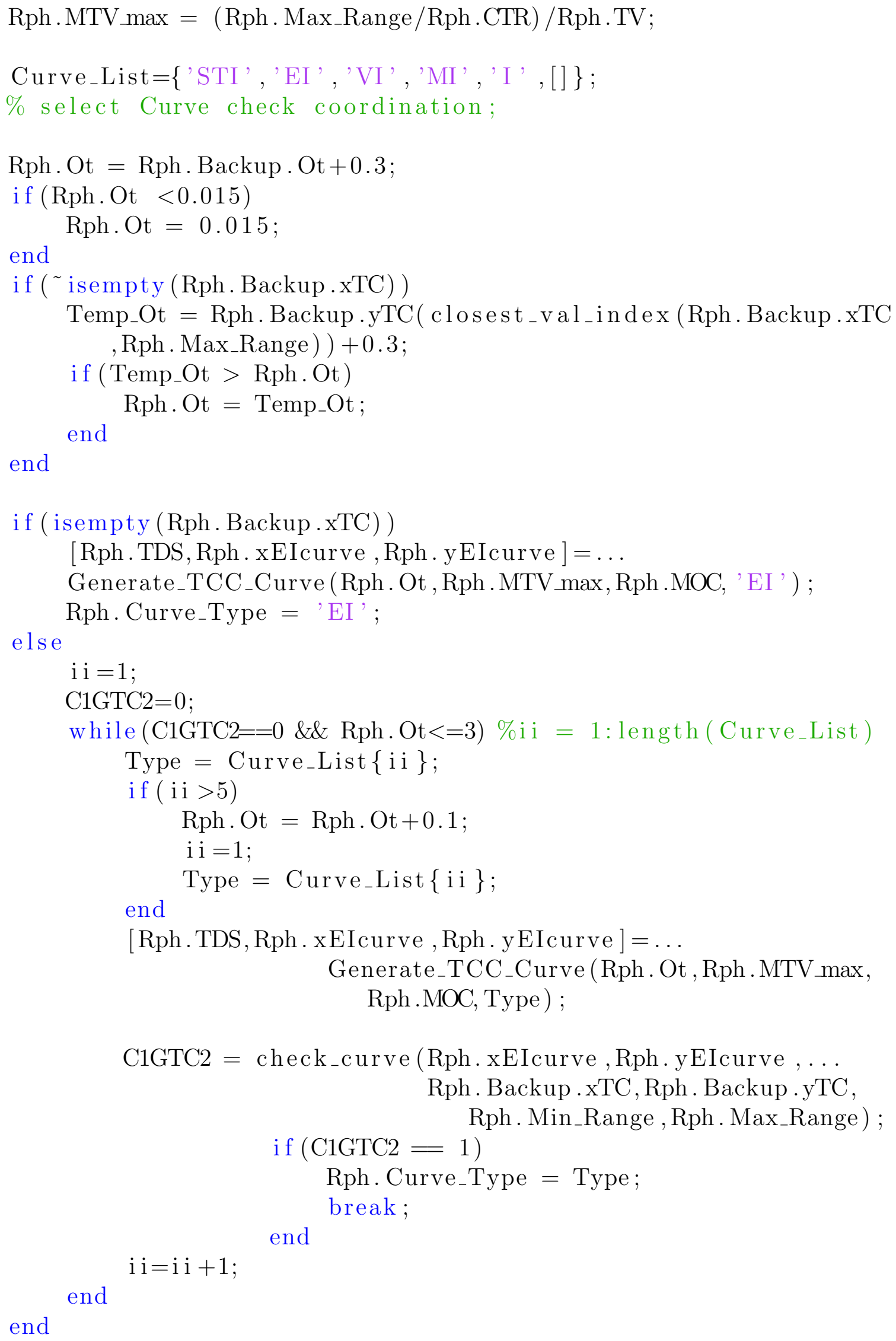




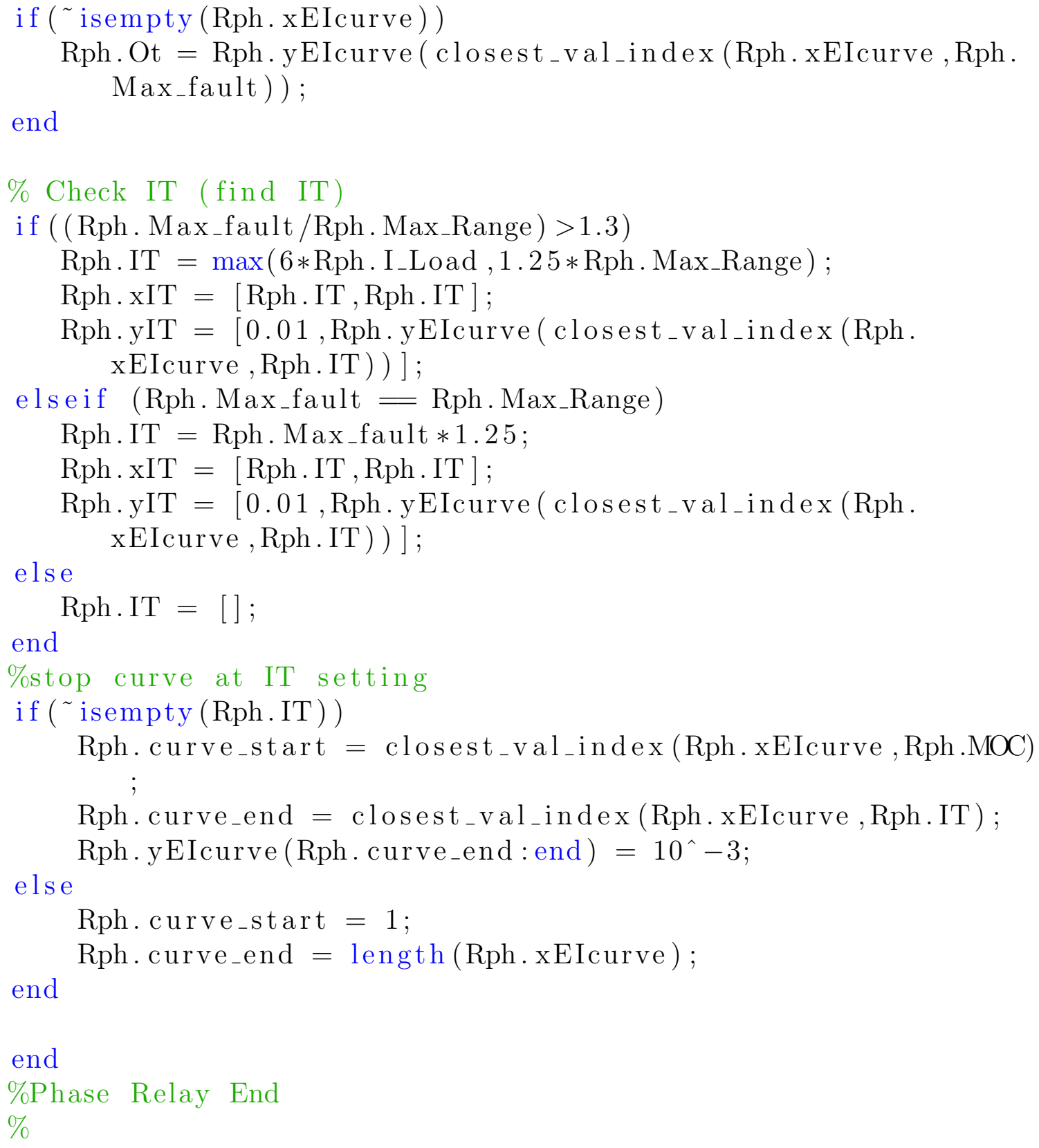




\section{Bibliography}

[1] Galen L. Barbose. U.S. Renewables Portfolio Standards: 2019 Annual Status Update. Tech. rep. Lawrence Berkeley National Laboratory, July 2019. URL: https://emp.lbl.gov/projects/renewables-portfolio.

[2] EIA. Annual Energy Outlook 2020. Tech. rep. U.S. Energy Information Administration, Jan. 2020. URL: https://www.eia.gov/outlooks/aeo/.

[3] S. S. Venkata et al. Microgrid Protection: Advancing the State of the Art. Tech. rep. Sandia National Laboratorie, Mar. 2019.

[4] Ahmad Razani Haron, Azah Mohamed, and Hussain Shareef. "A Review on Protection Schemes and Coordination Techniques in Microgrid System". In: Journal of Applied Sciences 12 (Feb. 2012), pp. 101-112. DOI: 10.3923/jas . 2012.101.112.

[5] J. Shiles et al. "Microgrid protection: An overview of protection strategies in North American microgrid projects". In: 2017 IEEE Power Energy Society General Meeting. July 2017, pp. 1-5. DOI: 10.1109/PESGM. 2017.8274519.

[6] M. A. Zamani, T. S. Sidhu, and A. Yazdani. "A Protection Strategy and Microprocessor-Based Relay for Low-Voltage Microgrids". In: IEEE Transactions on Power Delivery 26.3 (2011), pp. 1873-1883.

[7] H. Laaksonen, D. Ishchenko, and A. Oudalov. "Adaptive Protection and Microgrid Control Design for Hailuoto Island". In: IEEE Transactions on Smart Grid 5.3 (May 2014), pp. 1486-1493. ISSN: 1949-3061. DOI: 10.1109/TSG . 2013. 2287672.

[8] S. Brahma, N. Pragallapati, and M. Nagpal. "Protection of Islanded Microgrid Fed by Inverters". In: 2018 IEEE Power Energy Society General Meeting (PESGM). Aug. 2018, pp. 1-5. DOI: 10.1109/PESGM. 2018.8585892.

[9] S. Brahma. "Protection of Distribution System Islands Fed by Inverter-Interfaced Sources". In: 2019 IEEE Milan PowerTech. June 2019, pp. 1-6. DOI: 10.1109/ PTC . 2019.8810544.

[10] Nicholas Gurule et al. "Grid-forming Inverter Experimental Testing of Fault Current Contributions". In: IEEE Photovoltaic Specialists Conference (PVSC). June 2019. 
[11] S. Conti. "Protection issues and state of the art for microgrids with inverterinterfaced distributed generators". In: 2011 International Conference on Clean Electrical Power (ICCEP). 2011, pp. 643-647.

[12] Remus Teodorescu, Marco Liserre, and Pedro Rodriguez. Grid converters for photovoltaic and wind power systems. Wiley, 2011.

[13] J. Hernandez-Alvidrez et al. "PV-Inverter Dynamic Model Validation and Comparison Under Fault Scenarios Using a Power Hardware-in-the-Loop Testbed". In: 2018 IEEE 7th World Conference on Photovoltaic Energy Conversion (WCPEC). June 2018, pp. 1412-1417. DOI: 10.1109/PVSC. 2018.8547488.

[14] Amirnaser Yazdani. Voltage-sourced converters in power systems : modeling, control, and applications. Wiley, 2010.

[15] Javier Hernandez-Alvidrez et al. "Simulation of Grid-Forming Inverters Dynamic Models using a Power Hardware-in-the-Loop Testbed". In: 2019 IEEE Photovoltaic Specialists Conference (PVSC). June 2019.

[16] W. Du, R. H. Lasseter, and A. S. Khalsa. "Survivability of Autonomous Microgrid During Overload Events". In: IEEE Transactions on Smart Grid 10.4 (2019), pp. 3515-3524.

[17] A. Gkountaras, S. Dieckerhoff, and T. Sezi. "Evaluation of current limiting methods for grid forming inverters in medium voltage microgrids". In: 2015 IEEE Energy Conversion Congress and Exposition (ECCE). Sept. 2015, pp. 12231230. DOI: 10.1109/ECCE. 2015.7309831.

[18] "IEEE Standard for Interconnection and Interoperability of Distributed Energy Resources with Associated Electric Power Systems Interfaces". In: IEEE Std 1547-2018 (Revision of IEEE Std 1547-2003) (2018), pp. 1-138. DOI: 10.1109/ IEEESTD . 2018.8332112.

[19] S. M. Brahma, J. Trejo, and J. Stamp. "Insight into microgrid protection". In: IEEE PES Innovative Smart Grid Technologies, Europe. Oct. 2014, pp. 1-6. DOI: 10.1109/ISGTEurope.2014.7028820.

[20] T. Patel et al. "Adaptive Protection Scheme for a Real-World Microgrid with 100\% Inverter-Based Resources". In: 2020 IEEE Kansas Power and Energy Conference (KPEC). 2020, pp. 1-6. DOI: 10.1109/KPEC47870.2020.9167527. 